\title{
Phase Transformations in Electrically Conductive Ferromagnetic Shape-Memory Alloys, Their Thermodynamics and Analysis
}

\author{
TomÁš RoubíčeK \& GiUSEPPE TOMASSETTI
}

\author{
Communicated by F. Отто
}

\begin{abstract}
We derive a thermodynamically consistent general continuum-mechanical model describing mutually coupled martensitic and ferro/paramagnetic phase transformations in electrically-conductive magnetostrictive materials such as $\mathrm{NiMnGa}$. We use small-strain and eddy-current approximations, yet large velocities and electric current injected through the boundary are allowed. Fully nonlinear coupling of magneto-mechanical and thermal effects is considered. The existence of energy-preserving weak solutions is proved by showing convergence of timediscrete approximations constructed by a carefully designed semi-implicit regularized scheme.
\end{abstract}

\section{Introduction}

Shape-memory alloys are intermetallic compounds characterized by two phases with different crystallographic configurations, typically a high-symmetry austenitic phase and a low-symmetry martensitic phase that manifests itself in number of variants, see [4]. In these materials, large strains are attained by variant rearrangement. In ferromagnetic shape-memory alloys, variant rearrangement can be induced through an external magnetic field $[23,27,45,46]$. This makes ferromagnetic shapememory alloys potential candidates for smart materials, but also renders their mathematical modeling a non-trivial task, because of the complex interplay between martensitic and ferromagnetic microstructures.

In this paper we develop a general, rational, thermodynamically consistent continuum-mechanical model that is complex enough to take into account

This work was supported by the Italian INdAM-GNFM, and also in part by the grants 201/09/0917, 201/10/0357, and 201/12/0671 (GA ČR), LC 06052 (MŠMT ČR), by the institutional support RVO: 61388998 (ČR), as well as the CENTEM project no. CZ.1.05/21.00/03.0088 (within OPRDI) at New technologies research centre (ZĆU, Plzeň). 
martensitic and ferromagnetic phase transformations, and yet simple enough to be amenable to a rigorous mathematical analysis.

For the magnetic part of the modeling, we use the theory of micromagnetics [11], suitably extended to non-isothermal settings. Micromagnetics has been proven a very powerful tool for analytically deriving the macroscopic properties of magnetic samples, by establishing a connection between microstructure and macroscopic behavior both in the bulk [13] and in lower-dimensional structures, such as thin films [14]. One of the assumptions of standard micromagnetics is that the magnetization vector has a constant magnitude equal to the so-called saturation magnetisation. This assumption, also known as Heisenberg's constraint is, indeed, one of the features that makes the model interesting from the mathematical point of view. However, this constraint is relevant primarily in temperatures well below the Curie point (or better, even close to absolute zero) and must be dropped at temperatures around or above the Curie point, that is, in particular, if the ferro/para-magnetic transformation is considered, see [5]. Basic to our derivation of the evolution equations for the magnetization is the introduction of a system of microforces, which expend power over time variations of the magnetization. This approach, which opens the way to generalizations of standard micromagnetics [6], has been used in [15] to model magnetoelastic interactions in saturated ferromagnetic solids. A similar approach was followed in [25] to model microstructural evolution in ferromagnetic-shape memory alloys in the isothermal setting. In the non-isothermal setting, a thermodynamically-consistent model capable of describing the ferro/paramagnetic transition was derived in [35], accompanied by a proof of existence of weak solutions using the technical framework set forth in [41].

Several experiments have been reported $[42,50]$ in which $\mathrm{Ni}_{2} \mathrm{MnGa}$ samples are subject to mechanical vibrations up to frequencies of $10 \mathrm{MHz}$. These mechanical vibrations are expected to trigger fast magnetization oscillations, which in turn can produce eddy currents. As a result, phase transformation may be affected not only through magneto-mechanical coupling, but also through the produced Joule heat. This effect is also complicated because conductivity in shape-memory alloys depends not only on temperature, but mainly on the symmetry of the lattice [16, $21,48,51]$. For instance, variation of electric resistivity by tens of percents within the transformation of austenite to martensite or to R-phase has been experimentally documented $[19,28]$. For the above-mentioned reasons, we also include in our model the effects of eddy currents generated by the magnetization, as done in [40]. Altogether, these materials exhibit a very complex response that also includes mechanical-magnetical mutual interaction, see also [22,43], for example, which is only very schematically depicted in Fig. 1.

To make the model accessible to analysis, we work within the setting of small strains, and we also take into account surface-energy effects by including in the free energy a term dependent on the gradient of the strain. This is also known as the concept of so-called second-grade nonsimple materials (see, for example, [33,44]), alternatively also referred as the concept of hyper- or couple-stresses [34,47]. This gradient theory for small strains, together with the standard exchange-energy term for magnetization, enables us to treat nonconvex free energies $\varphi(\cdot, \theta)$, see (17), with (72b), a feature that is essential for describing phase transformation. Since this 


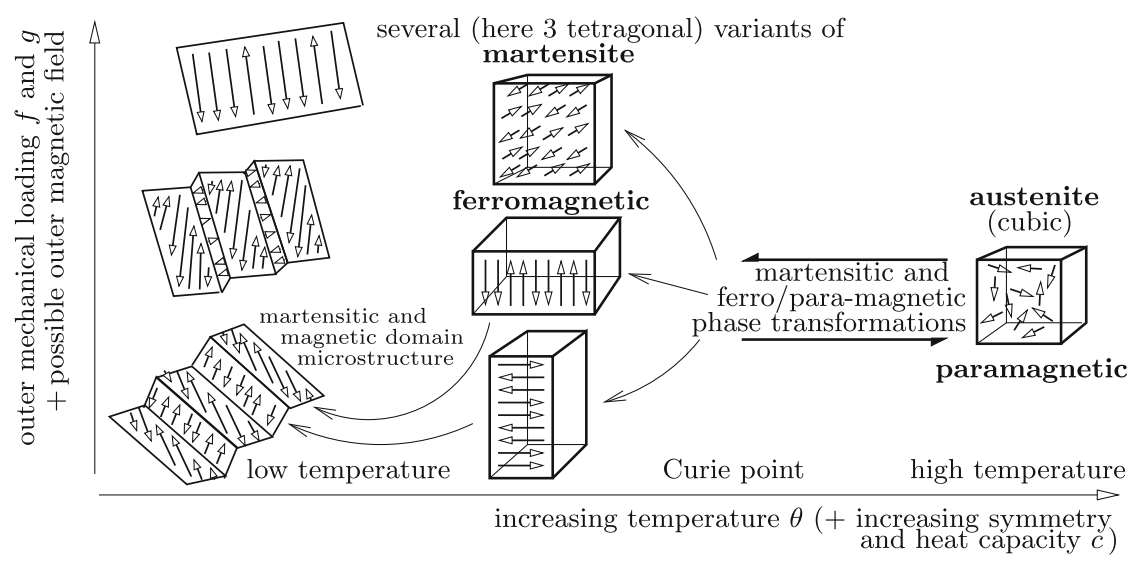

Fig. 1. Schematic illustration of very complex mechanical-magnetic-thermo response of a single-crystal of a magnetic shape-memory alloy like $\mathrm{Ni}_{2} \mathrm{MnGa}$ together with complicated self-accommodation of lower-symmetrical variants casting typically martensitic microstructures and magnetic fine domain structure. Important magneto-mechanical and dynamical interactions as well as variation of thermal conductivity $\mathbb{K}$ and electrical conductivity $\mathbb{S}$ are not depicted. Martensitic and ferro/para-magnetic transformations may undergo in different temperatures, depending on variation of stochiometric composition of this intermetalic alloy

capillarity effect may not be completely efficient from the energetic point of view, in the sense that the storage of energy into capillarity terms is likely to be accompanied by dissipation, we also introduce a viscous-like dissipation dependent on the time derivative of the gradient of the small-strain tensor. Consistently with the smallstrain and small-displacement assumption, we identify the reference and current configuration by assuming small displacements. We do not assume, however, small velocities, see Remark 1 in the next section, which may be important in certain applications involving high-frequency vibrations.

\section{The Model}

As the problem is very complex and involves a lot of mutually interacting fields, we necessarily must involve many symbols. For readers' convenience, we summarize the notation used in Table 1.

Let $\Omega \subset \mathbb{R}^{3}$ be a bounded Lipschitz domain occupied by the body in its reference configuration, and let $\Gamma=\partial \Omega$ denote its boundary. For the purpose of the derivation of the model we assume $\Omega$ smooth but, in fact, when the domain has sharp edges, hyperstresses may involve concentrated forces [33]. Dealing with these issues is beyond the scope of this paper, however. Letting the fixed time interval of interest $I=(0, T)$ and abbreviating $Q:=I \times \Omega$ and $\Sigma:=I \times \Gamma$, the unknown fields are $u: Q \rightarrow \mathbb{R}^{3}, m: Q \rightarrow \mathbb{R}^{3}, \theta: Q \rightarrow \mathbb{R}, h: Q \rightarrow \mathbb{R}^{3}$, and $e: Q \rightarrow \mathbb{R}^{3}$. In particular, $u(t, x)$ and $m(t, x)$, respectively, deliver the displacement of the material point $x$ at time $t$ and the magnetization density per unit referential volume at the same point and at the same time. The task we carry out throughout the rest of this section is the derivation of an initial-boundary-value problem, see (30)-(32) 
Table 1. Summary of the basic notation used throughout the paper

\begin{tabular}{ll}
\hline$u$ Displacement, & $\psi$ Free energy, \\
$m$ Magnetization, & $\varphi$ Coupling part of free energy, see (17), \\
$\theta$ Temperature, & $\phi(\cdot)=\varphi(\cdot, 0)$ \\
$h$ Magnetic field, & $s$ Entropy, \\
$e$ Electric field, & $\mu_{0}$ Vacuum permeability, \\
E, E $(u)$ Small-strain tensor, & $\mathbb{K}=\mathbb{K}(\mathfrak{q}, \theta)$ Thermal conductivity, \\
S Stress tensor, & $\mathbb{S}=\mathbb{S}(\mathfrak{q}, \theta)$ Electrical conductivity, \\
H Hyperstress (3rd-order) tensor, & $\varrho$ Mass density, \\
C Magnetic stress, & $c=c(\mathfrak{q}, \theta)$ Heat capacity, \\
$k$ Internal magnetic microforce, & $\gamma=\gamma(|m|)$ Effective gyromagnetic ratio, \\
$p$ External magnetic microforce, & $\mathbb{C}$ hpr Hyperelasticity tensor, \\
$\mathfrak{q}=(\mathrm{E}(u), m)$, & $\mathbb{D}$ Viscosity tensor, \\
$\mathfrak{p}=(\mathrm{S}, k)$, & $\mathbb{D}$ hpr Hyperviscosity tensor, \\
$\mathfrak{P}=(\mathbf{H}, \mathrm{C})$, & $\alpha$ Magnetic-dissipation constant, \\
$j$ Electric current, & $\lambda$ Magnetic exchange-energy constant, \\
$q$ Heat flux, & $\chi(\mathfrak{q})$ Phase-transformation indicator, \\
$q_{\mathrm{b}}$ Boundary heat flux, see(12), & $\zeta(\cdot) \geqq 0$ A degree-one homogeneous function, \\
$j_{\mathrm{b}}$ Surface current, see $(6)$ and $(31 \mathrm{e})$, & $f$ Bulk force (inertial and load), see $(1)$, \\
$h_{\mathrm{b}}$ An extension of $-v \times j_{\mathrm{b}}$ to $\Omega$, & $g$ Boundary traction, see $(2)$ with $(28 \mathrm{~b})$, \\
$\varepsilon$ Internal energy, & $g$ hpr Boundary hypertraction. \\
\hline
\end{tabular}

below, governing $(u, m, \theta, h, e)$. In particular, we shall obtain the first three partial differential equations of (30) by combining suitable balance equations, which we introduce now, with appropriate constitutive prescriptions, which we specify at a second stage.

To begin with, we introduce a stress $\mathrm{S}: Q \rightarrow \mathbb{R}_{\text {sym }}^{3 \times 3}$, a hyperstress $\mathbf{H}: Q \rightarrow$ $\mathbb{R}^{3 \times 3 \times 3}$, a bulk-force density $f: Q \rightarrow \mathbb{R}^{3}$, and a surface-force density $g: \Sigma: \rightarrow$ $\mathbb{R}^{3}$, and we lay down the force balance:

$$
\operatorname{div}(\mathbf{S}-\operatorname{div} \mathbf{H})+f=0,
$$

which we impose within the body. Since a hyperstress is involved, the force balance (1) is accompanied by two boundary conditions [34]: a traction condition and a hypertraction condition, respectively,

$$
(\mathbf{S}-\operatorname{div} \mathbf{H}) n-\operatorname{div}^{\mathrm{s}}(\mathbf{H} n)=g, \quad \mathbf{H}:(n \otimes n)=g^{\mathrm{hpr}},
$$

where $n$ denotes the outward unit normal. In writing (2), we maintain that the application of the third-order tensor $\mathbf{H}$ to the vector $n$ produces the second-order tensor with components $(\mathbf{H} n)_{i j}=\mathbf{H}_{i j k} n_{k}$. Moreover, div ${ }^{\mathrm{s}}$ is the surface-divergence operator, which may be introduced as follows [20]: given a vector field $v: \Gamma \rightarrow \mathbb{R}^{3}$, we extend it to a neighborhood of $\Gamma$, and we let its surface gradient be defined as $\nabla^{\mathrm{s}} v=\nabla v \mathbb{P}^{\mathrm{S}}$, where $\mathbb{P}^{\mathrm{S}}=\mathbb{I}-n \otimes n$ is the projector on the tangent space of $\Gamma$; we then let the surface divergence of $v$ be the scalar field $\operatorname{div}^{\mathrm{s}} v=\mathbb{P}^{\mathrm{s}}: \nabla^{\mathrm{s}} v=\operatorname{tr}\left(\mathbb{P}^{\mathrm{s}} \nabla v \mathbb{P}^{\mathrm{s}}\right)$. Given a tensor field $\mathbb{A}: \Gamma \rightarrow \mathbb{R}^{3 \times 3}$, we let $\operatorname{div}^{\mathrm{s}} \mathbb{A}: \Gamma \rightarrow \mathbb{R}^{3}$ be the unique vector field such that $\operatorname{div}^{\mathrm{s}}\left(\mathbb{A}^{T} a\right)=a \cdot \operatorname{div}^{\mathrm{s}} \mathbb{A}$ for all constant vector fields $a: \Gamma \rightarrow \mathbb{R}^{3}$. Furthermore, the symbol ":" denotes contraction between the last two indices. Thus, componentwise, the second condition in (2) reads: $\mathbf{H}_{i j k} n_{j} n_{k}=0$. 
As in [35], we introduce a magnetic microstress $\mathrm{C}: Q \rightarrow \mathbb{R}^{3 \times 3}$, an internal magnetic microforce $k: Q \rightarrow \mathbb{R}^{3}$, and an external magnetic microforce $p: Q \rightarrow$ $\mathbb{R}^{3}$ (a specification in terms on $u$ and $m$ will be presented later on); then, we write the magnetic microforce balance:

$$
\operatorname{div} \mathrm{C}-k+p=0
$$

along with the null-microtraction condition:

$$
\mathrm{C} n=0,
$$

which we assume to hold on the whole boundary. We do not envisage a Dirichlettype condition for $m$, since a direct physical interpretation of this condition is problematic, as well as an interpretation of a non-homogeneous microtraction condition.

Next, we write the system of Maxwell's equations in the so-called eddy-current approximation $[1,9]$ :

$$
\begin{aligned}
\mu_{0}(\dot{h}+\dot{m})+\operatorname{curl} e & =-\mu_{0}(\operatorname{div} \dot{u}) m-\mu_{0} \nabla m \dot{u}, \\
-\operatorname{curl} h+j & =0 .
\end{aligned}
$$

The interpretation of the term on right-hand side of the Faraday-Maxwell equation (5a) is provided in Remark 1 below. Equation (5b) follows from the AmpereMaxwell equation $\varepsilon_{0} \dot{e}-\operatorname{curl} h+j=0$ by neglecting the displacement current $\varepsilon_{0} \dot{e}$, where $\varepsilon_{0}$ denotes vacuum permittivity. As is well known, the standard Maxwell system is hyperbolic. Instead, with the eddy-current approximation, the ensuing evolution laws have a parabolic structure. We shall further discuss this point in Remark 4.

The solution of (5) in the whole space is seldom pursued. Typically, one restricts the domain of definition the electromagnetic fields to a bounded region surrounding the body of interest, and imposes appropriate insulating conditions at the boundary of the region. To avoid additional technical complication which would not alter the matter of our treatment, we shall assume that the electro-magnetic field in the surrounding region can be neglected, and we shall impose the following condition at the boundary:

$$
n \times h=j_{\mathrm{b}} .
$$

The above condition is understood in the sense of the following integration-by-parts formula [12]:

$$
\begin{aligned}
\int_{\Omega} \operatorname{curl} h \cdot e-h \cdot \operatorname{curl} e \mathrm{~d} x & =\int_{\Gamma}(v \times h) \cdot e \mathrm{~d} S \\
& =\int_{\Gamma} j_{\mathrm{b}} \cdot e \mathrm{~d} S=-\int_{\Gamma}\left(e \times h_{\mathrm{b}}\right) \cdot n \mathrm{~d} S,
\end{aligned}
$$

where the last equality follows from having defined:

$$
h_{\mathrm{b}}=-n \times j_{\mathrm{b}} .
$$


We denote by

$$
\mathrm{E}(u)=\operatorname{sym} \nabla u
$$

the small strain tensor. We shall write $\mathrm{E}$ in place of $\mathrm{E}(u)$ when appropriate. We shall also use the following shorthand notation:

$$
\mathfrak{q}:=(\mathrm{E}, m), \quad \mathfrak{p}:=(\mathrm{S}, k), \quad \mathfrak{P}:=(\mathbf{H}, \mathrm{C}) .
$$

Using the above notation, we can write the balance of internal energy in the form:

$$
\dot{\varepsilon}+\operatorname{div} q-j \cdot e-\mathfrak{p} \cdot \dot{\mathfrak{q}}-\mathfrak{P} \cdot \nabla \dot{\mathfrak{q}}=0
$$

We supplement this balance law with a prescription of the heat flux at the boundary:

$$
q \cdot n=q_{\mathrm{b}}
$$

Here $\varepsilon$ is the (specific) internal energy and $q$ is the heat flux. Letting $s$ be the (specific) entropy, the local form of the Clausius-Duhem inequality is:

$$
\theta \dot{s} \geqq q \cdot \nabla \theta-\operatorname{div} q
$$

By introducing the free energy:

$$
\psi:=\varepsilon-s \theta
$$

and by combining the Clausius-Duhem inequality with the balance of internal energy, we obtain the free-energy imbalance:

$$
\dot{\psi} \leqq-s \dot{\theta}-\theta^{-1} q \cdot \nabla \theta+j \cdot e+\mathfrak{p} \cdot \dot{\mathfrak{q}}+\mathfrak{P} \cdot \nabla \dot{\mathfrak{q}}
$$

Guided by (15), we assume that the free energy depends, at most, on $\mathfrak{q}=$ $(\mathrm{E}, m) \nabla \mathfrak{q}=(\nabla \mathbf{E}, \nabla m)$, and on $\theta$ :

$$
\psi=\psi(\mathfrak{q}, \nabla \mathfrak{q}, \theta)
$$

and we restrict our attention to constitutive dependencies of the form:

$$
\psi(\mathfrak{q}, \nabla \mathfrak{q}, \theta)=\varphi(\mathfrak{q}, \theta)+\frac{1}{2} \mathbb{C}_{\mathrm{hpr}} \nabla \mathrm{E}: \nabla \mathrm{E}+\frac{\lambda}{2}|\nabla m|^{2}
$$

The first term on the right-hand side of (17) incorporates temperature-dependent magnetoelastic coupling; specific choices for NiMnGa may be found in [24,52]. The second term takes into account interfacial energy between twin boundaries through the positive-definite sixth-order tensor $\mathbb{C}_{\mathrm{hpr}}$. The third term models the standard magnetic-exchange energy through a positive exchange parameter $\lambda$. A (by now) standard argument yields:

$$
s=s(\mathfrak{q}, \theta)=-\varphi_{\theta}^{\prime}(\mathfrak{q}, \theta) .
$$


In order to include dissipative effects in our model, we split $\mathfrak{p}$ and $\mathfrak{P}$ into their energetic and dissipative parts by writing:

$$
\begin{aligned}
\mathfrak{p} & =\mathfrak{p}^{\text {en }}+\mathfrak{p}^{\mathrm{di}}=\left(\mathrm{S}^{\mathrm{en}}, k^{\mathrm{en}}\right)+\left(\mathrm{S}^{\mathrm{di}}, k^{\mathrm{di}}\right), \\
\mathfrak{P} & =\mathfrak{P}^{\text {en }}+\mathfrak{P}^{\mathrm{di}}=\left(\mathbf{H}^{\mathrm{en}}, \mathrm{C}^{\mathrm{en}}\right)+\left(\mathbf{H}^{\mathrm{di}}, \mathrm{C}^{\mathrm{di}}\right),
\end{aligned}
$$

and by setting

$$
\begin{aligned}
& \mathfrak{p}^{\text {en }}=\mathfrak{p}^{\text {en }}(\mathfrak{q}, \nabla \mathfrak{q}, \theta)=\psi_{\mathfrak{q}}^{\prime}(\mathfrak{q}, \nabla \mathfrak{q}, \theta), \\
& \mathfrak{P}^{\text {en }}=\mathfrak{P}^{\text {en }}(\mathfrak{q}, \nabla \mathfrak{q}, \theta)=\psi_{\nabla \mathfrak{q}}^{\prime}(\mathfrak{q}, \nabla \mathfrak{q}, \theta) .
\end{aligned}
$$

As a consequence of (16)-(20), the force balance (1) and the magnetic microforce balance (3) become, respectively,

$$
\operatorname{div}\left(\varphi_{\mathrm{E}}^{\prime}(\mathrm{E}, m, \theta)+\mathrm{S}^{\mathrm{di}}-\operatorname{div}\left(\mathbb{C}_{\mathrm{hpr}} \nabla \mathrm{E}+\mathbf{H}^{\mathrm{di}}\right)\right)+f=0,
$$

and

$$
\lambda \Delta m-\varphi_{m}^{\prime}(\mathrm{E}, m, \theta)+\operatorname{div} \mathrm{C}^{\mathrm{di}}-k^{\mathrm{di}}+p=0 .
$$

Moreover, the balance of internal energy turns into a precursor of the heatconduction equation:

$$
c(\mathfrak{q}, \theta) \dot{\theta}+\operatorname{div} q=\xi+\theta \varphi_{\mathfrak{q}}^{\prime \prime}(\mathfrak{q}, \theta) \cdot \dot{\mathfrak{q}},
$$

where

$$
c(\mathfrak{q}, \theta)=-\theta \varphi_{\theta \theta}^{\prime \prime}(\mathfrak{q}, \theta)
$$

is the (specific) heat capacity, and

$$
\xi:=j \cdot \mathrm{e}+\mathfrak{p}^{\mathrm{di}} \cdot \dot{\mathfrak{q}}+\mathfrak{P}^{\mathrm{di}} \cdot \nabla \dot{\mathfrak{q}}
$$

is the (specific) dissipation rate. Likewise, the free-energy imbalance (15) turns into the reduced dissipation inequality:

$$
0 \leqq-\theta^{-1} q \cdot \nabla \theta+\xi
$$

Our next task is to establish, in a manner consistent with (24), appropriate constitutive restrictions on $\left(q, j, \mathfrak{p}^{\mathrm{di}}, \mathfrak{P}^{\mathrm{di}}\right)$. To this aim, we introduce the total dissipation potential

$$
\begin{aligned}
\zeta_{\text {tot }}(\mathfrak{q}, \theta ; \nabla \theta, e, \dot{\mathfrak{q}}, \nabla \dot{\mathfrak{q}})= & \frac{1}{2} \mathbb{K}(\mathfrak{q}, \theta) \nabla \theta \cdot \nabla \theta+\mathbb{S}(\mathfrak{q}, \theta) e \cdot e+\frac{1}{2} \mathbb{D} \dot{\mathrm{E}}: \dot{\mathrm{E}} \\
& +\frac{1}{2} \mathbb{D}_{\mathrm{hpr}} \nabla \dot{\mathrm{E}}: \nabla \dot{\mathrm{E}}+\frac{1}{2} \alpha|\dot{m}|^{2}+\zeta\left(\chi_{\mathrm{E}}^{\prime}(\mathfrak{q}) \dot{\mathrm{E}}+\chi_{m}^{\prime}(\mathfrak{q}) \dot{m}\right),
\end{aligned}
$$

and we require:

$$
\left(q, j, \mathfrak{p}^{\mathrm{di}}, \mathfrak{P}^{\mathrm{di}}\right) \in \partial_{(-\nabla \theta, e, \dot{\mathfrak{q}}, \nabla \dot{\mathfrak{q}})} \zeta_{\text {tot }}(\mathfrak{q}, \theta ; \nabla \theta, e, \dot{\mathfrak{q}}, \nabla \dot{\mathfrak{q}}) .
$$


The constitutive prescription (26) is equivalent to:

$$
\begin{aligned}
q & =-\mathbb{K}(\mathfrak{q}, \theta) \nabla \theta, \\
j & =\mathbb{S}(\mathfrak{q}, \theta) \mathrm{e}, \\
\mathrm{S}^{\mathrm{di}} & =\mathbb{D} \dot{\mathrm{E}}+\chi_{\mathrm{E}}^{\prime}(\mathfrak{q})^{\top} \omega, \\
\mathbf{H}^{\mathrm{di}} & =\mathbb{D}_{\mathrm{hpr}} \nabla \dot{\mathrm{E}}, \\
k^{\mathrm{di}} & =\alpha \dot{m}+\chi_{\mathrm{m}}^{\prime}(\mathfrak{q})^{\top} \omega, \\
\mathrm{C}^{\mathrm{di}} & =0, \\
\omega & \in \partial \zeta(\dot{\chi}),
\end{aligned}
$$

with $\dot{\chi}=\frac{\partial}{\partial t}(\chi(\mathrm{E}(u), m))=\chi_{\mathrm{E}}^{\prime}(\mathfrak{q}) \mathrm{E}(\dot{u})+\chi_{m}^{\prime}(\mathfrak{q}) \dot{m}$. With (27) at hand, we can write the dissipation-rate $(23)$ as:

$$
\xi=\mathbb{D} \mathrm{E}(\dot{u}): \mathrm{E}(\dot{u})+\mathbb{D}_{\mathrm{hpr}} \nabla \mathrm{E}(\dot{u}): \nabla \mathrm{E}(\dot{u})+\mathbb{S}(\mathfrak{q}, \theta) e \cdot e+\alpha|\dot{m}|^{2}+\zeta(\dot{\chi}) .
$$

The phenomenological relations (27a) and (27b) generalize the standard Fourier's and Ohm's laws by including possible dependence on strain, magnetization and temperature of the thermal conductivity tensor $\mathbb{K}(\mathfrak{q}, \theta)$ and of the electrical conductivity tensor $\mathbb{S}(\mathfrak{q}, \theta)$. Moreover, $\mathbb{D}$ (resp. $\mathbb{D}_{\mathrm{hpr}}$ ) is a positive-definite fourth-order (resp. sixth-order) tensor whose components $[\mathbb{D}]_{i j k l}$ satisfy $[\mathbb{D}]_{i j k l}=\mathbb{D}_{j i k l}=\mathbb{D}_{i j l k}$ (resp. $\left.\left[\mathbb{D}_{\mathrm{hpr}}\right]_{i j k l m n}=\left[\mathbb{D}_{\mathrm{hpr}}\right]_{j i k l m n}=\left[\mathbb{D}_{\mathrm{hpr}}\right]_{i j k m l n}\right)$. Furthermore, $\alpha$ is the standard micromagnetic dissipation constant [17]. Finally, $\zeta: \mathbb{R}^{L} \rightarrow \mathbb{R}^{+}:=[0,+\infty)$ is a degree-1 homogeneous function and $\chi: \mathbb{R}^{3 \times 3} \times \mathbb{R}^{3} \rightarrow \mathbb{R}^{L}$ is a "phase indicator". A typical modeling assumption is that the range of $\chi$ is the Gibbs' simplex $\triangle:=\left\{\left(\chi_{1}, \ldots, \chi_{L}\right) \in\left(\mathbb{R}^{+}\right)^{L} ; \sum_{i=1}^{L} \chi_{i}=1\right\}$ and values of $\chi$ are around particular vertices of $\triangle$ if $\mathfrak{q}$ is (in the vicinity of) a particular phase, which makes it possible to distinguish the $L+1$ phases. Then $\zeta$ determines the energy needed for transformation between particular phases. These functions incorporate, at the same time, phenomenological rate-independent dissipation due to phase transformation $[2,3,32,36,40]$ and magnetic-pinning effects $[41,49]$. The phase indicator $\chi$ has a meaning similar to the phase field used in [26]. The main difference is that a phase indicator depends on other variables, whereas a phase field is an independent quantity.

It remains for us to prescribe the volume-force density $f$ in (1), the surfaceforce density $g$ in (2), and the bulk term $p$ in (3). To this aim, we introduce the following splitting:

$$
\begin{aligned}
f & =f_{0}+f_{\text {in }}+f_{\mathrm{em}}, \\
g & =g_{0}+g_{\text {in }}+g_{\mathrm{em}}, \\
g^{\mathrm{hpr}} & =g_{0}^{\mathrm{hpr}}+g_{\text {in }}+g_{\mathrm{em}}, \\
p & =p_{0}+p_{\text {in }}+p_{\mathrm{em}} .
\end{aligned}
$$

Here, a subscript " 0 " means that the corresponding field is a given datum. The subscripts "in" and "em" stand for "inertial" and "electromagnetic", respectively, 
and denote terms that may depend on $u, m, \theta, h$, and $e$; for these terms, we make the following constitutive assumptions (see Remark 2):

$$
\begin{aligned}
& f_{\text {in }}=-\varrho \ddot{u}, g_{\text {in }}=0, g_{\text {in }}^{\mathrm{hpr}}=0, p_{\text {in }}=\frac{m \times \dot{m}}{\gamma(|m|)}, \\
& f_{\mathrm{em}}=-\mu_{0} \nabla h^{\top} m, g_{\mathrm{em}}=\mu_{0}(m \cdot h) n, g_{\mathrm{em}}^{\mathrm{hpr}}=0, p_{\mathrm{em}}=\mu_{0} h .
\end{aligned}
$$

The constitutive prescription for $p_{\text {in }}$ incorporates gyromagnetic inertia in the evolution equation for $m$. For a discussion, see [35]. Our last step consists in combining (21) and the Maxwell system (5) with the constitutive prescriptions (27), (28)-(29). By doing so, we arrive at the following system of equations and inclusion in $\Omega$ to be solved by the six-tuple $(u, m, e, h, \theta, \omega)$ :

$$
\begin{aligned}
& \varrho \ddot{u}-\operatorname{div}\left(\varphi_{\mathrm{E}}^{\prime}(\mathrm{E}(u), m, \theta)+\mathbb{D} \mathrm{E}(\dot{u})+\chi_{\mathrm{E}}^{\prime}(E(u), m)^{\top} \omega\right. \\
& \left.\quad-\operatorname{div}\left(\mathbb{C}_{\mathrm{hpr}} \nabla \mathrm{E}(u)+\mathbb{D}_{\mathrm{hpr}} \nabla \mathrm{E}(\dot{u})\right)\right)=f_{0}-\mu_{0} \nabla h^{\top} m \\
& \alpha \dot{m}-\frac{m \times \dot{m}}{\gamma(|m|)}-\lambda \Delta m+\varphi_{m}^{\prime}(\mathrm{E}(u), m, \theta)=p_{0}+\mu_{0} h-\chi_{m}^{\prime}(E(u), m)^{\top} \omega \\
& c(\mathrm{E}(u), m, \theta) \dot{\theta}-\operatorname{div}(\mathbb{K}(\mathrm{E}(u), m, \theta) \nabla \theta)=\zeta(\dot{\chi})+\mathbb{S}(\mathrm{E}(u), m, \theta) e: e \\
& \quad+\mathbb{D} \mathrm{E}(\dot{u}): \mathrm{E}(\dot{u})+\mathbb{D}_{\mathrm{hpr}} \nabla \mathrm{E}(\dot{u}) \vdots \nabla \mathrm{E}(\dot{u})+\alpha|\dot{m}|^{2} \\
& \quad+\theta \varphi_{\mathrm{E} \theta}^{\prime \prime}(\mathrm{E}(u), m, \theta): \mathrm{E}(\dot{u})+\theta \varphi_{m \theta}^{\prime \prime}(\mathrm{E}(u), m, \theta) \cdot \dot{m} \\
& \mu_{0}(\dot{h}+\dot{m})+\operatorname{curl} e=-\mu_{0}(\operatorname{div} \dot{u}) m-\mu_{0} \nabla m \dot{u} \\
& \operatorname{curl} h-\mathbb{S}(\mathrm{E}(u), m, \theta) e=0 \\
& \omega \in \partial \zeta(\dot{\chi})
\end{aligned}
$$

where $f_{0}: Q \rightarrow \mathbb{R}^{3}, g_{0}: \Sigma \rightarrow \mathbb{R}^{3}, h_{\mathrm{b}}: \Sigma \rightarrow \mathbb{R}^{2}$ are prescribed bulk and boundary data. The boundary conditions are:

$$
\begin{aligned}
& \left(\varphi_{\mathrm{E}}^{\prime}(\mathrm{E}(u), m, \theta)+\mathbb{D} \mathrm{E}(\dot{u})+\chi_{\mathrm{E}}^{\prime}(\mathrm{E}(u), m)^{\top} \omega-\operatorname{div} \mathbf{H}\right) n \\
& \quad-\operatorname{div}^{\mathrm{s}}(\mathbf{H} n)-\mu_{0}(m \cdot h) n=g_{0}, \\
& \mathbf{H}:(n \otimes n)=g_{0}^{\mathrm{hpr}} \quad \text { with } \mathbf{H}=\mathbb{C}_{\mathrm{hpr}} \nabla \mathrm{E}(u)+\mathbb{D}_{\mathrm{hpr}} \nabla \mathrm{E}(\dot{u}), \\
& \frac{\partial m}{\partial n}=0, \\
& \mathbb{K}(E(u), m, \theta) \frac{\partial \theta}{\partial n}=q_{\mathrm{b}}, \\
& n \times h=j_{\mathrm{b}},
\end{aligned}
$$

where $j_{b}: \Sigma \rightarrow \mathbb{R}^{3}$ is a prescribed surface electric current, assumed to be tangent to the surface $\Gamma$ for all $t$, see the qualification (50) together with (72r), later. As initial conditions, we take:

$$
\begin{aligned}
& u(0, \cdot)=u_{0}, \quad \dot{u}(0, \cdot)=v_{0}, \quad m(0, \cdot)=m_{0}, \quad h(0, \cdot)=h_{0}, \\
& \theta(0, \cdot)=\theta_{0} .
\end{aligned}
$$


Remark 1. (The convective term.) Let us explain the convective term on the righthand side of (5a). In our picture, $m$ is the density of magnetization per unit volume in the reference configuration. This quantity should not be confused with the spatial density $m_{s}$ that is actually measured in the physical space. In terms of $m_{s}$, the first Maxwell equation reads:

$$
\mu_{0}\left(\dot{h}+\dot{m}_{S}\right)+\operatorname{curl} e=0 .
$$

The relation between $m_{s}$ and $m$ is

$$
\operatorname{det}(\mathbb{I}+\nabla u(t, x)) m_{s}(t, x+u(t, x))=m(t, x),
$$

where we maintain that $m(t, x)=0$ for $x \notin \Omega$. Differentiating both sides of (33) with respect to $t$ yields

$$
\operatorname{det}(\mathbb{I}+\nabla u)\left(\dot{m}_{s}+(\mathbb{I}+\nabla u)^{-\top}(\operatorname{div} \dot{u}) m_{s}+\nabla m_{s} \dot{u}\right)=\dot{m},
$$

where for brevity we omit independent variables. At this point, we introduce the small-displacement approximation $x+u \approx x$, which entails $\nabla u \approx 0, m_{s} \approx m$, and $\nabla m_{s} \approx \nabla m$, so that (34) can be approximated by:

$$
\dot{m}_{s} \approx \dot{m}-(\operatorname{div} \dot{u}) m-\nabla m \dot{u},
$$

whence the convective term in (5a). Again, we note that $\dot{u}$ is not considered small, which is a case of small but very fast mechanical vibrations in some experiments on frequencies about $10 \mathrm{MHz}$ or more.

Remark 2. (Bulk and surface forces.) Our constitutive choices for the surface tractions/hypertractions imply that the sum of the electromagnetic power expended on the continuum and of the flux of the Poynting vector $e \times h=e \times h_{\mathrm{b}}$ at the boundary compensate for the rate of change of magnetic energy. Indeed, let us note the following identity:

$$
\begin{aligned}
\int_{\Omega} \mu_{0}((\operatorname{div} \dot{u}) m+\nabla m \dot{u}) \cdot h \mathrm{~d} x & =\int_{\Omega} \mu_{0} \operatorname{div}(m \otimes \dot{u}) \cdot h \mathrm{~d} x \\
& =\int_{\Omega} \mu_{0}(\operatorname{div}((\dot{u} \otimes m) h)-(m \otimes \dot{u}): \nabla h) \mathrm{d} x \\
& =\int_{\Gamma} \mu_{0}(m \cdot h)(\dot{u} \cdot n) \mathrm{d} S-\int_{\Omega} \mu_{0} \nabla h^{\top} m \cdot \dot{u} \mathrm{~d} x \\
& =\int_{\Gamma} g_{\mathrm{em}} \cdot \dot{u} \mathrm{~d} S+\int_{\Omega} f_{\mathrm{em}} \cdot \dot{u} \mathrm{~d} x
\end{aligned}
$$

Observe that (35) holds formally since, as we shall see in the next section, $\nabla h$ is not defined as a function; in fact, for the sake of mathematical analysis, in the weak formulation we will express the force $\mu_{0} \nabla h^{\top} m$ rather as $\mu_{0}(\nabla m)^{\top} h+\mu_{0} \nabla(h \cdot m)$, see (70a). Using (5a), (7), and (35), we have 


$$
\begin{aligned}
\int_{\Omega} \mu_{0} \dot{h} \cdot h \mathrm{~d} x= & -\int_{\Omega} \operatorname{curl} e \cdot h-\int_{\Omega} \mu_{0}(\dot{m}+(\operatorname{div} \dot{u}) m+\nabla m \dot{u}) \cdot h \mathrm{~d} x \\
= & -\int_{\Omega} e \cdot j \mathrm{~d} x-\int_{\Gamma}\left(e \times h_{\mathrm{b}}\right) \cdot n \mathrm{~d} S \\
& +\int_{\Omega}\left(p_{\mathrm{em}} \cdot \dot{m}+f_{\mathrm{em}} \cdot \dot{u}\right) \mathrm{d} x-\int_{\Gamma} g_{\mathrm{em}} \cdot \dot{u} \mathrm{~d} S,
\end{aligned}
$$

where $h_{\mathrm{b}}$ denotes a prescribed magnetic field on the boundary, see (8); in fact, the normal component of $h_{\mathrm{b}}$ is irrelevant, and $h_{\mathrm{b}}$ is related with $j_{\mathrm{b}}$ occurring in (31e) via (50) below. Then, we get the following balance statement:

$$
\begin{aligned}
& \int_{\Omega} \underbrace{p_{\mathrm{em}} \cdot \dot{m}}_{\begin{array}{c}
\text { Electro-Magnetic } \\
(\text { E-M) power }
\end{array}}+\underbrace{f_{\mathrm{em}} \cdot \dot{u}}_{\begin{array}{c}
\text { power of E-M } \\
\text { bulk force }
\end{array}}+\underbrace{e \cdot j}_{\begin{array}{c}
\text { Joule } \\
\text { heat }
\end{array}} \mathrm{d} x \\
& +\int_{\Gamma} \underbrace{g_{\mathrm{em}} \cdot \dot{u}}+\underbrace{\left(e \times h_{\mathrm{b}}\right) \cdot n} \mathrm{~d} S+\frac{\mathrm{d}}{\mathrm{d} t} \int_{\Omega} \underbrace{\frac{\mu_{0}}{2}|h|^{2}} \mathrm{~d} x=0 . \\
& \begin{array}{c}
\text { power of } \\
\text { E-M traction }
\end{array} \quad \text { magnetic }
\end{aligned}
$$

We remark that the balance of electro-magnetic energy involves not only a bulk electromagnetic force $f_{\mathrm{em}}$, but also an electromagnetic traction $g_{\mathrm{em}}$ at the boundary. Notice, however, that there is no appearance of $\frac{\partial}{\partial n} \dot{u}$ in the boundary integral in (37), consistently with the choice $g_{\mathrm{em}}^{\mathrm{hpr}}=0$ in $(29 \mathrm{~b})$.

Also, we can derive the balance of total energy, that is, internal + magnetic + kinetic, see (39) below. Indeed, integrating the energy balance (11) over $\Omega$ and using (1)-(4), we get

$$
\frac{\mathrm{d}}{\mathrm{d} t} \int_{\Omega} \varepsilon=\int_{\Gamma} g \cdot \dot{u}+g^{\mathrm{hpr}} \cdot \frac{\partial \dot{u}}{\partial n}+q_{\mathrm{b}} \mathrm{d} S+\int_{\Omega} j \cdot e+f \cdot \dot{u}+p \cdot \dot{m} \mathrm{~d} x .
$$

Since the inertial force compensates the rate of kinetic energy, that is,

$$
\int_{\Omega} \underbrace{f_{\text {in }} \cdot \dot{u}}_{\substack{\text { inertial } \\
\text { power }}} \mathrm{d} x+\frac{\mathrm{d}}{\mathrm{d} t} \int_{\Omega} \underbrace{\frac{\varrho}{2}|\dot{u}|^{2}}_{\begin{array}{c}
\text { kinetic } \\
\text { energy }
\end{array}} \mathrm{d} x=0,
$$

we obtain, using also the decompositions (28) and the balance (37),

$$
\begin{aligned}
& \frac{\mathrm{d}}{\mathrm{d} t} \int_{\Omega} \varepsilon+\frac{\mu_{0}}{2}|h|^{2}+\frac{\varrho}{2}|\dot{u}|^{2} \mathrm{~d} x \\
& \quad=\int_{\Gamma} g_{0} \cdot \dot{u}+g_{0}^{\mathrm{hpr}} \cdot \frac{\partial \dot{u}}{\partial n}+q_{\mathrm{b}}+j_{\mathrm{b}} \cdot e \mathrm{~d} S+\int_{\Omega} f_{0} \cdot \dot{u} \mathrm{~d} x,
\end{aligned}
$$

where (31e) has been used. Another estimate can be obtained by testing (1) and (3) by $\dot{u}$ and $\dot{m}$, respectively, and using the traction conditions (2) and (4). This procedure yields:

$$
\int_{\Omega} \mathfrak{p} \cdot \dot{\mathfrak{q}}+\mathfrak{P} \cdot \nabla \dot{\mathfrak{q}} \mathrm{d} x=\int_{\Omega} f \cdot \dot{u}+p \cdot \dot{m} \mathrm{~d} x+\int_{\Gamma} g \cdot \dot{u}+g^{\mathrm{hpr}} \cdot \frac{\partial \dot{u}}{\partial n} \mathrm{~d} S .
$$


Again, taking into account the decomposition (28), and the identities (38)-(37), we obtain:

$$
\begin{aligned}
& \int_{\Omega}(\mathfrak{p} \cdot \dot{\mathfrak{q}}+\mathfrak{P} \cdot \nabla \dot{\mathfrak{q}}) \mathrm{d} x+\frac{\mathrm{d}}{\mathrm{d} t} \int_{\Omega} \frac{\varrho}{2}|\dot{u}|^{2}+\frac{\mu_{0}}{2}|h|^{2} \mathrm{~d} x \\
& =\int_{\Omega} f_{0} \cdot \dot{u}-j \cdot e \mathrm{~d} x+\int_{\Gamma} g_{0} \cdot \dot{u}+g_{0}^{\mathrm{hpr}} \cdot \frac{\partial \dot{u}}{\partial n}+j_{\mathrm{b}} \cdot e \mathrm{~d} S .
\end{aligned}
$$

Using (19)-(20), we further obtain

$$
\begin{aligned}
\int_{\Omega} \varphi_{\mathfrak{q}}^{\prime}(\mathfrak{q}, \nabla \theta) \cdot \dot{\mathfrak{q}}+\mathfrak{p}^{\mathrm{di}} \cdot \dot{\mathfrak{q}}+\mathfrak{P}^{\mathrm{di}} \cdot \nabla \dot{\mathfrak{q}} \mathrm{d} x \\
+\frac{\mathrm{d}}{\mathrm{d} t} \int_{\Omega} \frac{1}{2} \mathbb{C}_{\mathrm{hpr}} \nabla \mathrm{E}: \nabla \mathrm{E}+\frac{\lambda}{2}|\nabla m|^{2}+\frac{\varrho}{2}|\dot{u}|^{2}+\frac{\mu_{0}}{2}|h|^{2} \mathrm{~d} x \\
\quad=\int_{\Omega} f_{0} \cdot \dot{u}-j \cdot e \mathrm{~d} x+\int_{\Gamma} g_{0} \cdot \dot{u}+g_{0}^{\mathrm{hpr}} \cdot \frac{\partial \dot{u}}{\partial n}+j_{\mathrm{b}} \cdot e \mathrm{~d} S .
\end{aligned}
$$

Eventually, owing to the constitutive equations (27), we obtain the identity:

$$
\begin{aligned}
\int_{\Omega} \varphi_{q}^{\prime}(\mathfrak{q}, \theta) \cdot \dot{\mathfrak{q}}+\zeta\left(\chi_{\mathfrak{q}}^{\prime}(\mathfrak{q}) \cdot \dot{\mathfrak{q}}\right)+\mathbb{S}(\mathfrak{q}, \theta) e: e+\mathbb{D} \dot{\mathrm{E}}: \dot{\mathrm{E}}+\mathbb{D}_{\mathrm{hpr}} \nabla \dot{\mathrm{E}}: \nabla \dot{\mathrm{E}}+\alpha|\dot{m}|^{2} \mathrm{~d} x \\
+\frac{\mathrm{d}}{\mathrm{d} t} \int_{\Omega} \frac{1}{2} \mathbb{C}_{\mathrm{hpr}} \nabla \mathrm{E}: \nabla \mathrm{E}+\frac{\lambda}{2}|\nabla m|^{2}+\frac{\varrho}{2}|\dot{u}|^{2}+\frac{\mu_{0}}{2}|h|^{2} \mathrm{~d} x \\
\quad=\int_{\Omega} f_{0} \cdot \dot{u} \mathrm{~d} x+\int_{\Gamma} g_{0} \cdot \dot{u}+g_{0}^{\mathrm{hpr}} \cdot \frac{\partial \dot{u}}{\partial n}+j_{\mathrm{b}} \cdot e \mathrm{~d} S .
\end{aligned}
$$

Remark 3. (Estimates with enthalpy transformation.) In the next section we shall use the function

$$
\hat{c}(\mathfrak{q}, \theta):=\int_{0}^{\theta} c(\mathfrak{q}, \Theta) \mathrm{d} \Theta .
$$

We thus have, using the definition (22) of heat capacity, and entropy,

$$
\begin{aligned}
\hat{c}(\mathfrak{q}, \theta) & =-\int_{0}^{\theta} \theta \varphi_{\theta \theta}^{\prime \prime}(\mathfrak{q}, \Theta) \mathrm{d} \Theta=\int_{0}^{\theta} \varphi_{\theta}^{\prime}(\mathfrak{q}, \Theta) \mathrm{d} \Theta-\theta \varphi_{\theta}^{\prime}(\mathfrak{q}, \theta) \\
& =\varphi(\mathfrak{q}, \theta)-\phi(\mathfrak{q})+s(\mathfrak{q}, \theta) \theta
\end{aligned}
$$

where we have defined

$$
\phi(\mathfrak{q})=\varphi(\mathfrak{q}, 0)
$$

Recalling the relation (14) between internal energy and free energy, we conclude that the following important decomposition holds:

$$
\varepsilon(\mathfrak{q}, \nabla \mathfrak{q}, \theta)=\hat{c}(\mathfrak{q}, \theta)+\phi(\mathfrak{q})+\frac{1}{2} \mathbb{C}_{\mathrm{hpr}} \nabla \mathrm{E}: \nabla \mathrm{E}+\frac{1}{2} \lambda|\nabla m|^{2}
$$


Thus, defining

$$
\begin{aligned}
\Phi(u, v, m, h):= & \int_{\Omega} \frac{\varrho}{2}|v|^{2}+\phi(\mathrm{E}(u), m) \\
& +\frac{1}{2} \mathbb{C}_{\mathrm{hpr}} \nabla \mathrm{E}(u) \vdots \nabla \mathrm{E}(u)+\frac{\lambda}{2}|\nabla m|^{2}+\frac{\mu_{0}}{2}|h|^{2} \mathrm{~d} x,
\end{aligned}
$$

we obtain the following formal estimate by integrating (39) over time:

$$
\begin{gathered}
\Phi(u(t), \dot{u}(t), m(t), h(t))+\int_{\Omega} \hat{c}(\mathfrak{q}(t), \theta(t)) \mathrm{d} x=\Phi\left(u_{0}, v_{0}, m_{0}, h_{0}\right) \\
+\int_{\Omega} \hat{c}\left(\mathfrak{q}_{0}, \theta_{0}\right) \mathrm{d} x+\int_{Q} f_{0} \cdot \dot{u} \mathrm{~d} x \mathrm{~d} t+\int_{\Sigma} g_{0} \cdot \dot{u}+q_{\mathrm{b}}+j_{\mathrm{b}} \cdot e \mathrm{~d} S \mathrm{~d} t
\end{gathered}
$$

see also its time-discrete version (117). Also, a different version of (41) can be easily obtained by noticing that

$$
\begin{aligned}
\varphi_{\mathfrak{q}}^{\prime}(\mathfrak{q}, \theta) & =\phi^{\prime}(\mathfrak{q})+\int_{0}^{\theta} \varphi_{\theta \mathfrak{q}}^{\prime \prime}(\mathfrak{q}, \Theta) \mathrm{d} \Theta=\phi^{\prime}(\mathfrak{q})-\int_{0}^{\theta} \theta \varphi_{\theta \theta \mathfrak{q}}^{\prime \prime \prime}(\mathfrak{q}, \Theta) \mathrm{d} \Theta+\theta \varphi_{\theta \mathfrak{q}}^{\prime \prime}(\mathfrak{q}, \theta) \\
& =\phi^{\prime}(\mathfrak{q})+\int_{0}^{\theta} c_{\mathfrak{q}}^{\prime}(\mathfrak{q}, \Theta) \mathrm{d} \Theta+\theta \varphi_{\theta \mathfrak{q}}^{\prime \prime}(\mathfrak{q}, \theta) .
\end{aligned}
$$

Thus, on setting $A(\mathfrak{q}, \theta)=\int_{0}^{\theta} c_{\mathfrak{q}}^{\prime}(\mathfrak{q}, \Theta) \mathrm{d} \Theta+\theta \varphi_{\theta \mathfrak{q}}^{\prime \prime}(\mathfrak{q}, \theta)$ we obtain:

$$
\varphi_{\mathfrak{q}}^{\prime}(\mathfrak{q}, \theta)=\phi^{\prime}(\mathfrak{q})+A(\mathfrak{q}, \theta)
$$

Consequently, (41) can also be written as:

$$
\begin{aligned}
& \frac{\mathrm{d}}{\mathrm{d} t} \int_{\Omega} \phi(\mathfrak{q})+\frac{1}{2} \mathbb{C}_{\mathrm{hpr}} \nabla \mathrm{E}: \nabla \mathrm{E}+\frac{\lambda}{2}|\nabla m|^{2}+\frac{\varrho}{2}|\dot{u}|^{2}+\frac{\mu_{0}}{2}|h|^{2} \mathrm{~d} x \\
& \quad+\int_{\Omega} A(\mathfrak{q}, \theta) \cdot \dot{\mathfrak{q}}+\zeta\left(\chi_{\mathfrak{q}}^{\prime}(\mathfrak{q}) \cdot \dot{\mathfrak{q}}\right)+\mathbb{S}(\mathfrak{q}, \theta) e: e+\mathbb{D} \dot{\mathrm{E}}: \dot{\mathrm{E}}+\mathbb{D}_{\mathrm{hpr}} \nabla \dot{\mathrm{E}}: \nabla \dot{\mathrm{E}}+\alpha|\dot{m}|^{2} \mathrm{~d} x \\
& \quad=\int_{\Omega} f_{0} \cdot \dot{u} \mathrm{~d} x+\int_{\Gamma} g_{0} \cdot \dot{u}+j_{\mathrm{b}} \cdot e \mathrm{~d} S
\end{aligned}
$$

The last term, although physically relevant, cannot be directly used to obtain a priori estimates because $e$ is not expected to have well defined traces on $\Gamma$. The natural strategy for treating this term and the boundary condition (31e) is to introduce an auxiliary field $h_{\mathrm{b}}$ on the domain $\Omega$ satisfying

$$
n \times\left. h_{\mathrm{b}}\right|_{\Gamma}=j_{\mathrm{b}} .
$$

Using (7) with $h=h_{\mathrm{b}}$ and (30d), we can replace the surface integral in the righthand side of (49) with volume integrals, namely

$$
\begin{aligned}
& \int_{\Gamma} j_{\mathrm{b}} \cdot e \mathrm{~d} S=\int_{\Gamma}\left(n \times h_{\mathrm{b}}\right) \cdot e \mathrm{~d} S=\int_{\Omega} \operatorname{curl} h_{\mathrm{b}} \cdot e-\operatorname{curl} e \cdot h_{\mathrm{b}} \mathrm{d} x \\
& =\int_{\Omega} \operatorname{curl} h_{\mathrm{b}} \cdot e+\mu_{0} \dot{h} \cdot h_{\mathrm{b}}+\mu_{0} \dot{m} \cdot h_{\mathrm{b}}+\mu_{0}(\operatorname{div} \dot{u}) m \cdot h_{\mathrm{b}}+\mu_{0}(\nabla m \dot{u}) \cdot h_{\mathrm{b}} \mathrm{d} x .
\end{aligned}
$$

Later, our estimation strategy will be based on a discrete analog of (49)-(51), see (109). 
Remark 4. (The parabolic structure of (30d)-(30e).) In the special case $\mathbb{S}(\mathfrak{q}, \theta)=$ $\sigma \mathbb{I}$, the constitutive equation (27b) becomes

$$
j=\sigma e .
$$

Then, the unknowns $h, e$ in the system (5) can be converted into a diffusion equation in the unknown $h$. Indeed, substituting the constitutive equation (52) into (5b), dividing by $\sigma$ and taking the curl of both sides, we obtain

$$
e=\sigma^{-1} \operatorname{curl}^{2} h,
$$

which substituted into (5a) yields

$$
\dot{h}+\left(\mu_{0} \sigma\right)^{-1} \operatorname{curl}^{2} h=-\dot{m}-r,
$$

where we have abbreviated $r=(\operatorname{div} \dot{u}) m-\nabla m \dot{u}$. Using the identity $\operatorname{curl}^{2} h=$ $\nabla$ div $h-\Delta h$, we can rewrite (53) as

$$
\dot{h}-\left(\mu_{0} \sigma\right)^{-1} \Delta h=-\dot{m}-r-\left(\mu_{0} \sigma\right)^{-1} \nabla \operatorname{div} h .
$$

If the initial data satisfy the usual assumption $\operatorname{div}\left(h_{0}+m_{0}\right)=0$, by applying the operator $\nabla$ div to both sides of (53) and integrating with respect to time, we obtain

$$
\nabla \operatorname{div} h=-\nabla \operatorname{div}\left(m-\int_{0}^{t} r \mathrm{~d} t\right)
$$

thus (54) becomes

$$
\dot{h}-\left(\mu_{0} \sigma\right)^{-1} \Delta h=-\dot{m}-r-\left(\mu_{0} \sigma\right)^{-1} \nabla \operatorname{div}\left(m-\int_{0}^{t} r \mathrm{~d} t\right),
$$

which reveals the parabolic character of the eddy-current approximation.

Remark 5. (Cross-coupling effects.) Our model can be extended to account for Seebeck and Peltier effects by generalizing the choice (25) of the total dissipation potential $\zeta_{\text {tot }}$ as

$$
\begin{aligned}
\zeta_{\text {tot }}(\nabla \theta, e, \mathfrak{q}, \nabla \mathfrak{q}, \dot{\mathfrak{q}}, \nabla \dot{\mathfrak{q}})= & \frac{1}{2}\left(\begin{array}{c}
\nabla \theta \\
e
\end{array}\right)\left(\begin{array}{cc}
-\mathbb{K}(\mathfrak{q}, \theta) & \mathbb{B}_{\mathrm{S}}(\mathfrak{q}, \theta) \\
\mathbb{B}_{\mathrm{P}}(\mathfrak{q}, e) & \mathbb{S}(\mathfrak{q}, \theta)
\end{array}\right)\left(\begin{array}{c}
\nabla \theta \\
e
\end{array}\right)+\frac{1}{2} \dot{\mathrm{E}}: \mathbb{D} \dot{\mathrm{E}} \\
& +\frac{1}{2} \nabla \dot{\mathrm{E}}: \mathbb{D}_{\mathrm{hpr}} \nabla \dot{\mathrm{E}}+\frac{\alpha}{2}|\dot{m}|^{2}+\zeta\left(\chi_{\mathrm{E}}^{\prime}(\mathfrak{q}) \dot{\mathrm{E}}+\chi_{m}^{\prime}(\mathfrak{q}) \dot{m}\right) .
\end{aligned}
$$

Here, $\mathbb{B}_{\mathrm{S}}$ is the Seebeck-effect coupling tensor and $\mathbb{B}_{\mathrm{P}}$ is the Peltier-effect coupling tensor [31], satisfying the Onsager's reciprocity condition [29,30],

$$
\mathbb{B}_{\mathrm{S}}(\mathfrak{q}, \theta)=\theta \mathbb{B}_{\mathrm{P}}^{\top}(\mathfrak{q}, \theta) .
$$

This would augment (27a) and (27b), respectively, as:

$$
\begin{aligned}
q & =-\mathbb{K}(\mathfrak{q}, \theta) \nabla \theta+\mathbb{B}_{\mathrm{S}}(\mathfrak{q}, \theta) e, \\
j & =\mathbb{S}(\mathfrak{q}, \theta) \mathrm{e}+\mathbb{B}_{\mathrm{P}}(\mathfrak{q}, e) \nabla \theta .
\end{aligned}
$$

From the analytical point of view, however, the ensuing technical complications are issues that fall out of the scope of the present paper. 
Remark 6. (Injection of electric current.) In order to better understand the role of the boundary condition (6), a formal computation is of help. To this aim, let us note that, since $j_{\mathrm{b}}$ is tangential to the surface, we have:

$$
\int_{\Gamma} j_{\mathrm{b}} \cdot \nabla v+\operatorname{div}^{\mathrm{s}}\left(j_{\mathrm{b}}\right) v \mathrm{~d} S=0
$$

for every scalar test function $v$. Then, using (5b), along with the identities $\operatorname{div} \operatorname{curl} h \equiv 0$ and curl $\nabla v=0$, and with the integration-by-parts formula (7), we obtain

$$
\begin{aligned}
\int_{\Gamma}(j \cdot n) v \mathrm{~d} S & =\int_{\Gamma}(\operatorname{curl} h \cdot n) v \mathrm{~d} S=\int_{\Omega} \operatorname{curl} h \cdot \nabla v+(\operatorname{div} \operatorname{curl} h) v \mathrm{~d} x \\
& =\int_{\Omega} h \cdot \operatorname{curl} \nabla v+\int_{\Gamma}(n \times h) \cdot \nabla v \mathrm{~d} S \\
& =\int_{\Gamma} j_{\mathrm{b}} \cdot \nabla v \mathrm{~d} S=-\int_{\Gamma} \operatorname{div}^{\mathrm{s}}\left(j_{\mathrm{b}}\right) v \mathrm{~d} S
\end{aligned}
$$

By the arbitrariness of $v$, we have

$$
j \cdot n=-\operatorname{div}^{\mathrm{s}}\left(j_{\mathrm{b}}\right) .
$$

In other words, by specifying the tangential surface current $j_{\mathrm{b}}$, we can also impose the normal current pumped in/out of the conductor. Note that the physical dimension of the surface current $j_{\mathrm{b}}$ is $\mathrm{A} / \mathrm{m}$, so that $\operatorname{div}^{\mathrm{s}}\left(j_{\mathrm{b}}\right)$ has physical dimension $\mathrm{A} / \mathrm{m}^{2}$ and thus (60), indeed, prescribes the electric-current density. Another option to pump electric current in/out the body would be due to the right-hand side of (30e) on some (presumably thin) layer $\Omega_{0}$ adjacent to the boundary on which, however, the conductivity $\mathbb{S}$ would have to be considered zero, see $[9, \S 1.2 .2]$. Then, in fact, $e$ would be controlled in $L^{2}\left(\Omega \backslash \Omega_{0}\right)$ rather than in $L^{2}(\Omega)$.

\section{Weak Solutions}

Although we have already applied a number of simplifications (small strains, eddy-current approximation of the Maxwell system, and cross-effects neglected), the analysis of the system (30) is still complicated in many aspects, in particular by the $L^{1}$-structure of heat sources and by the non-integrable term $c(\mathfrak{q}, \theta) \dot{\theta}$. Standardly, if $\varphi$ would take a partly linearized form $\varphi(\mathfrak{q}, \theta)=\varphi_{0}(\theta)+\varphi_{1}(\mathfrak{q})+\theta \varphi_{2}(\mathfrak{q})$, the entropy separates the variables, that is, $s=s(\mathfrak{q}, \theta)=-\varphi_{0}^{\prime}(\theta)-\varphi_{2}(\mathfrak{q})$, and the heat capacity is independent of $\mathfrak{q}$, that is, $\mathrm{E}$ and $m$. Then the term $c(\theta) \dot{\theta}$ can be easily integrated as $\frac{\partial}{\partial t} \hat{c}(\theta)$, where $\hat{c}(\theta)$ is a primitive function of $c$, and one can introduce a simple substitution $\vartheta=\hat{c}(\theta)$, called the enthalpy transformation.

As devised in [38] and already announced in Remark 3, we can enhance this procedure for our case, too. The enhanced enthalpy transformation requires gradient theory for mechanical/magnetic variables, but we have used this theory, anyhow, in our model. This procedure is based on an elementary calculus:

$$
c(\mathfrak{q}, \theta) \dot{\theta}=\frac{\partial \hat{c}(\mathfrak{q}, \theta)}{\partial t}-c_{1}(\mathfrak{q}, \theta): \dot{\mathbf{E}}-c_{2}(\mathfrak{q}, \theta) \cdot \dot{m},
$$


where $\hat{c}$ is from (42) and where

$$
c_{1}(\mathfrak{q}, \theta)=\int_{0}^{\theta} c_{\mathrm{E}}^{\prime}(\mathfrak{q}, \Theta) \mathrm{d} \Theta \quad \text { and } \quad c_{2}(\mathfrak{q}, \theta)=\int_{0}^{\theta} c_{m}^{\prime}(\mathfrak{q}, \Theta) \mathrm{d} \Theta .
$$

We introduce the new variable

$$
\vartheta=\hat{c}(\mathfrak{q}, \theta) .
$$

We assume that $\hat{c}(\mathfrak{q}, \cdot)$ is invertible, and we denote its inverse by $\mathcal{T}(\mathfrak{q}, \cdot)$. Then, on setting

$$
\begin{aligned}
\mathscr{K}_{0}(\mathfrak{q}, \vartheta): & =\mathbb{K}(\mathfrak{q}, \mathscr{T}(\mathfrak{q}, \vartheta)) \mathscr{T}_{\vartheta}^{\prime}(\mathfrak{q}, \vartheta), \\
\mathscr{K}_{1}(\mathfrak{q}, \vartheta): & =\mathbb{K}(\mathfrak{q}, \mathscr{T}(\mathfrak{q}, \vartheta)) \mathscr{T}_{\mathrm{E}}^{\prime}(\mathfrak{q}, \vartheta), \\
\mathscr{K}_{2}(\mathfrak{q}, \vartheta): & =\mathbb{K}(\mathfrak{q}, \mathscr{T}(\mathfrak{q}, \vartheta)) \mathscr{T}_{m}^{\prime}(\mathfrak{q}, \vartheta), \\
\mathscr{S}(\mathfrak{q}, \vartheta): & =\mathbb{S}(\mathfrak{q}, \mathscr{T}(\mathfrak{q}, \vartheta)), \\
\mathscr{A}_{1}(\mathfrak{q}, \vartheta): & =\mathscr{T}(\mathfrak{q}, \vartheta) \varphi_{\mathrm{E} \theta}^{\prime \prime}(\mathfrak{q}, \mathscr{T}(\mathfrak{q}, \vartheta))+c_{1}(\mathfrak{q}, \mathscr{T}(\mathfrak{q}, \vartheta)), \\
\mathscr{A}_{2}(\mathfrak{q}, \vartheta): & =\mathscr{T}(\mathfrak{q}, \vartheta) \varphi_{m}^{\prime \prime}(\mathfrak{q}, \mathscr{T}(\mathfrak{q}, \vartheta))+c_{2}(\mathfrak{q}, \mathscr{T}(\mathfrak{q}, \vartheta)), \\
\sigma_{\mathrm{E}}(\mathfrak{q}, \vartheta): & =\varphi_{\mathrm{E}}^{\prime}(\mathfrak{q}, \mathscr{T}(\mathfrak{q}, \vartheta)), \\
\sigma_{m}(\mathfrak{q}, \vartheta): & =\varphi_{m}^{\prime}(\mathfrak{q}, \mathscr{T}(\mathfrak{q}, \vartheta)) .
\end{aligned}
$$

we have

$$
\begin{aligned}
\mathbb{K}(\mathfrak{q}, \theta) \nabla \theta & =\mathbb{K}(\mathfrak{q}, \mathscr{T}(e, \vartheta)) \nabla \mathscr{T}(\mathfrak{q}, \vartheta) \\
& =\mathscr{K}_{0}(\mathfrak{q}, \vartheta) \nabla \vartheta+\mathscr{K}_{1}(\mathfrak{q}, \vartheta) \nabla \mathrm{E}+\mathscr{K}_{2}(\mathfrak{q}, \vartheta) \nabla m .
\end{aligned}
$$

Thus, in terms of the 6-tuple $(u, m, \vartheta, e, h, \omega)$, the system (30) transforms to the following six equations/inclusion:

$$
\begin{aligned}
& \varrho \ddot{u}-\operatorname{div}\left(\sigma_{\mathrm{E}}(\mathrm{E}(u), m, \vartheta)+\mathbb{D} \mathrm{E}(\dot{u})+\chi_{\mathrm{E}}^{\prime}(\mathrm{E}(u), m)^{\top} \omega\right. \\
& \left.-\operatorname{div}\left(\mathbb{C}_{\mathrm{hpr}} \nabla \mathrm{E}(u)+\mathbb{D}_{\mathrm{hpr}} \nabla \mathrm{E}(\dot{u})\right)\right)=f_{0}-\mu_{0} \nabla h^{\top} m, \\
& \alpha \dot{m}-\frac{m \times \dot{m}}{\gamma(|m|)}-\lambda \Delta m+\sigma_{m}(\mathrm{E}(u), m, \vartheta)=p_{0}+\mu_{0} h-\chi_{m}^{\prime}(\mathrm{E}(u), m)^{\top} \omega, \\
& \dot{\vartheta}-\operatorname{div}\left(\mathscr{K}_{0}(\mathrm{E}(u), m, \vartheta) \nabla \vartheta+\mathscr{K}_{1}(\mathrm{E}(u), m, \vartheta) \nabla \mathrm{E}(u)+\mathscr{K}_{2}(\mathrm{E}(u), m, \vartheta) \nabla m\right)=\bar{\xi} \\
& \text { with } \bar{\xi}:=\zeta\left(\chi_{\mathrm{E}}^{\prime}(\mathrm{E}(u), m) \mathrm{E}(\dot{u})+\chi_{m}^{\prime}(\mathrm{E}(u), m) \dot{m}\right)+\mathscr{S}(\mathrm{E}(u), m, \vartheta) e \cdot e \\
& +\mathbb{D E}(\dot{u}): \mathrm{E}(\dot{u})+\mathbb{D}_{\mathrm{hpr}} \nabla \mathrm{E}(\dot{u}): \nabla \mathrm{E}(\dot{u})+\alpha|\dot{m}|^{2} \\
& +\mathscr{A}_{1}(\mathrm{E}(u), m, \vartheta): \mathrm{E}(\dot{u})+\mathscr{A}_{2}(\mathrm{E}(u), m, \vartheta) \cdot \dot{m}, \\
& \mu_{0}(\dot{h}+\dot{m})+\operatorname{curl} e=-\mu_{0} \nabla m \dot{u}-\mu_{0}(\operatorname{div} \dot{u}) m, \\
& \operatorname{curl} h-\mathscr{S}(\mathrm{E}(u), m, \vartheta) e=0 \text {, } \\
& \omega \in \partial \zeta\left(\chi_{\mathrm{E}}^{\prime}(\mathrm{E}(u), m) \mathrm{E}(\dot{u})+\chi_{m}^{\prime}(\mathrm{E}(u), m) \dot{m}\right),
\end{aligned}
$$


completed by the boundary conditions $(31 \mathrm{~b}, \mathrm{c}, \mathrm{d}, \mathrm{e})$ and

$$
\begin{aligned}
& \left(\sigma_{\mathrm{E}}(\mathrm{E}(u), m, \vartheta)+\mathbb{D} \mathrm{E}(\dot{u})+\chi_{\mathrm{E}}^{\prime}(\mathrm{E}(u), m)^{\top} \omega-\operatorname{div} \mathbf{H}\right) \cdot v \\
& -\operatorname{div}^{\mathrm{s}}(\mathbf{H} \cdot v)-\mu_{0}(h \cdot m) v=g_{0}, \\
& \mathscr{K}_{0}(\mathrm{E}(u), m, \vartheta) \frac{\partial \vartheta}{\partial n}+\mathscr{K}_{1}(\mathrm{E}(u), m, \vartheta) \frac{\partial \mathrm{E}(u)}{\partial n}+\mathscr{K}_{2}(\mathrm{E}(u), m, \vartheta) \frac{\partial m}{\partial n}=q_{\mathrm{b}}
\end{aligned}
$$

and the initial conditions (32a) completed by

$$
\vartheta(0, \cdot)=\hat{c}\left(\mathrm{E}\left(u_{0}\right), m_{0}, \theta_{0}\right) .
$$

We will use standard notation for function space, namely spaces of continuous $\mathbb{R}^{l}$-valued functions $C\left(\bar{\Omega} ; \mathbb{R}^{l}\right)$, continuously differentiable functions $C^{1}\left(\bar{\Omega} ; \mathbb{R}^{l}\right)$, Lebesgue spaces $L^{p}\left(\Omega ; \mathbb{R}^{l}\right)$, Sobolev spaces $W^{k, p}\left(\Omega ; \mathbb{R}^{l}\right)$ and Bochner spaces of $X$-valued functions $L^{p}(I ; X)$. Moreover, we denote by $\mathrm{B}(\bar{I} ; X), \mathrm{BV}(\bar{I} ; X)$, and $C_{\mathrm{w}}(\bar{I} ; X)$ the Banach spaces of functions from $\bar{I}:=[0, T]$ to $X$ that are, respectively, bounded measurable, have a bounded variation, and are weakly continuous; note that all these functions are defined everywhere on $\bar{I}$. We will use the notation $p^{\prime}=p /(p-1)$ for the conjugate exponent to $p$. Instead of $u(t, \cdot)$ or $\mathfrak{q}(t, \cdot)$ or $\vartheta(t, \cdot)$ etc., we will write briefly $u(t)$ or $\mathfrak{q}(t)$ or $\vartheta(t)$ etc., respectively. Also, we make use of the Banach space

$$
L_{\text {curl }}^{p, q}\left(\Omega ; \mathbb{R}^{3}\right):=\left\{v \in L^{p}\left(\Omega ; \mathbb{R}^{3}\right) ; \operatorname{curl} v \in L^{q}\left(\Omega ; \mathbb{R}^{3}\right)\right\},
$$

and, for $p=q$, we shall write simply $L_{\text {curl }}^{p}\left(\Omega ; \mathbb{R}^{3}\right)$. Occasionally, we will also use

$$
L_{\text {curl }, 0}^{p}\left(\Omega ; \mathbb{R}^{3}\right):=\left\{v \in L_{\text {curl }}^{p}\left(\Omega ; \mathbb{R}^{3}\right): v \times\left. n\right|_{\Gamma}=0\right\} .
$$

The weak formulation of the heat equation (64c) can be obtained by multiplying it formally by $v$, integrating over $Q$, and applying Green's formula. Also for (64a,e,f), we apply integration in time. We shall also assume, for simplicity,

$$
g_{0}^{\mathrm{hpr}}=0, \quad p_{0}=0 .
$$

Thus, also using $h_{\mathrm{b}}$ from (50), we arrive to the following definition:

Definition 1. (Weak solutions.) We say that the 6-tuple $(u, m, \vartheta, h, e, \omega)$ such that

$$
\begin{aligned}
& u \in W^{1,2}\left(I ; \mathrm{W}^{2,2}\left(\Omega ; \mathbb{R}^{3}\right)\right) \cap \mathrm{W}^{1, \infty}\left(I ; L^{2}\left(\Omega ; \mathbb{R}^{3}\right)\right), \\
& m \in \mathrm{W}^{1,2}\left(I ; L^{2}\left(\Omega ; \mathbb{R}^{3}\right)\right) \cap L^{\infty}\left(I ; \mathrm{W}^{1,2}\left(\Omega ; \mathbb{R}^{3}\right)\right), \\
& \vartheta \in L^{r}\left(I ; \mathrm{W}^{1, r}(\Omega)\right) \cap L^{\infty}\left(I ; L^{1}(\Omega)\right) \quad \text { with } r \in[1,5 / 4), \\
& h-h_{\mathrm{b}} \in L^{\infty}\left(I ; L_{\text {curl }, 0}^{2}\left(\Omega ; \mathbb{R}^{3}\right)\right), \\
& e \in L^{2}\left(Q ; \mathbb{R}^{3}\right), \\
& \omega \in L^{\infty}\left(Q ; \mathbb{R}^{L}\right)
\end{aligned}
$$

with $\vartheta \geqq 0$ almost everywhere in $Q$, is a weak solution to system (64) with boundary/initial conditions (31c,d,e) and (65) if: 
(i) $u(0)=u_{0}$ and, for all $v \in W^{1,2}\left(I ; L^{2}\left(\Omega ; \mathbb{R}^{3}\right)\right) \cap L^{2}\left(I ; W^{2,2}\left(\Omega ; \mathbb{R}^{3}\right)\right)$, it holds that

$$
\begin{aligned}
\int_{Q} & \left(\mathbb{D} \mathrm{E}(\dot{u})+\sigma_{\mathrm{E}}(\mathrm{E}(u), m, \vartheta)\right): \mathrm{E}(v)+\left(\mathbb{D}_{\mathrm{hpr}} \nabla \mathrm{E}(\dot{u})+\mathbb{C}_{\text {hyper }} \nabla \mathrm{E}(u)\right) \vdots \nabla \mathrm{E}(v) \\
& +\omega: \chi_{\mathrm{E}}^{\prime}(\mathrm{E}(u), m) \mathrm{E}(v)-\varrho \dot{u} \cdot \dot{v} \mathrm{~d} x \mathrm{~d} t+\int_{\Omega} \varrho \dot{u}(T) \cdot v(T) \mathrm{d} x \\
= & \int_{\Omega} \varrho v_{0} \cdot v(0) \mathrm{d} x+\int_{Q}\left(f_{0}+\mu_{0}(\nabla m)^{\top} h\right) \cdot v \\
& +\mu_{0}(h \cdot m) \operatorname{div} v \mathrm{~d} x \mathrm{~d} t+\int_{\Sigma} g_{0} \cdot v \mathrm{~d} S \mathrm{~d} t
\end{aligned}
$$

(ii) $m(0, \cdot)=m_{0}$ and for all $z \in \mathrm{W}^{1,2}\left(\Omega ; \mathbb{R}^{3}\right)$ and at almost all time instances $t$,

$$
\begin{aligned}
& \int_{\Omega} \lambda \nabla m: \nabla z+\left(\sigma_{m}(\mathrm{E}(u), m, \vartheta)+\alpha \dot{m}-\frac{m \times \dot{m}}{\gamma(|m|)}-\mu_{0} h\right) \cdot z \\
& \quad+\omega \cdot \chi_{m}^{\prime}(\mathrm{E}(u), m) z \mathrm{~d} x=0 ;
\end{aligned}
$$

(iii) for all $z \in C^{1}(\bar{Q})$ such that $z(T, \cdot)=0$,

$$
\begin{aligned}
\int_{Q} & \left(\mathscr{K}_{0}(\mathrm{E}(u), m, \vartheta) \nabla \vartheta \cdot \nabla z+\left(\mathscr{K}_{1}(\mathrm{E}(u), m, \vartheta) \nabla \mathrm{E}(u)\right) \cdot \nabla z\right. \\
+ & \left.\left(\mathscr{K}_{2}(\mathrm{E}(u), m, \vartheta) \nabla m\right) \cdot \nabla z-\vartheta \frac{\partial z}{\partial t}-\bar{\xi} z\right) \mathrm{d} x \mathrm{~d} t \\
= & \int_{\Omega} \vartheta_{0} z(0) \mathrm{d} x+\int_{\Sigma} q_{\mathrm{b}} z \mathrm{~d} x \mathrm{~d} t
\end{aligned}
$$

where $\bar{\xi}$ is from $(64 \mathrm{c})$;

(iv) for all $z \in C^{1}\left(\bar{I} ; L^{2}\left(\Omega ; \mathbb{R}^{3}\right)\right) \cap C\left(\bar{I} ; L_{\text {curl }, 0}^{2}\left(\Omega ; \mathbb{R}^{3}\right)\right)$ such that $z(T, \cdot)=0$,

$$
\begin{aligned}
& \int_{Q} \frac{e \cdot \operatorname{curl} z}{\mu_{0}}-(h+m) \cdot \dot{z}-\nabla m \dot{u} \\
& \quad z-(\operatorname{div} \dot{u}) m z \mathrm{~d} x \mathrm{~d} t=\int_{\Omega}\left(h_{0}+m_{0}\right) \cdot z(0, \cdot) \mathrm{d} x
\end{aligned}
$$

(v) for all $z \in C\left(\bar{I} ; L_{\text {curl }, 0}\left(\Omega ; \mathbb{R}^{3}\right)\right)$

$$
\int_{Q} h \cdot \operatorname{curl} z-\mathscr{S}(\mathrm{E}(u), m, \vartheta) e \cdot z \mathrm{~d} x \mathrm{~d} t=0
$$

(vi) and eventually $\omega$ from (i) and (ii) satisfies, for all $z \in L^{2}\left(Q ; \mathbb{R}^{L}\right)$,

$$
\begin{aligned}
& \int_{Q} \xi(z)-\omega:\left(z-\chi_{\mathrm{E}}^{\prime}(\mathrm{E}(u), m) \mathrm{E}(\dot{u})-\chi_{m}^{\prime}(\mathrm{E}(u), m) \dot{m}\right) \mathrm{d} x \mathrm{~d} t \\
& \geq \int_{Q} \xi\left(\chi_{\mathrm{E}}^{\prime}(\mathrm{E}(u), m) \mathrm{E}(\dot{u})+\chi_{m}^{\prime}(\mathrm{E}(u), m) \dot{m}\right) \mathrm{d} x \mathrm{~d} t
\end{aligned}
$$


For the justification of the particular terms coupling $h, m$, and $\dot{u}$, see (35). Note that $(70 \mathrm{~d}, \mathrm{e})$ arises from $(64 \mathrm{~d}, \mathrm{e})$ by the curl-formula (7) with general test functions $L_{\text {curl }, 0}\left(\Omega ; \mathbb{R}^{3}\right)$ vanishing the boundary integrals.

We first state our main existence result. For this, we first summarize the assumptions. We use the abbreviation

$$
\mathscr{K}=\left(\mathscr{K}_{1}, \mathscr{K}_{2}\right) \quad \text { and } \quad \mathscr{A}=\left(\mathscr{A}_{1}, \mathscr{A}_{2}\right) \text {. }
$$

We then impose the following assumptions, formulated in terms of the transformed data:

$$
\begin{aligned}
& \phi(\cdot):=\varphi(\cdot, 0): \mathbb{R}_{\text {sym }}^{3 \times 3} \times \mathbb{R}^{3} \rightarrow \mathbb{R}^{+} \text {is continuously differentiable, and } \\
& \exists C_{\phi} \in \mathbb{R} \varepsilon>0: \phi(\cdot)+C_{\phi}|\cdot|^{2} \text { is convex (that is, so-called semiconvexity of } \phi \text { ), } \\
& \phi(\mathfrak{q}) \leqq C_{\phi}\left(1+|\mathfrak{q}|^{6}\right), \quad\left|\phi^{\prime}(\mathfrak{q})\right| \leqq C_{\phi}\left(1+|\mathfrak{q}|^{5-\varepsilon}\right), \\
& \left|\phi_{m}^{\prime}\right|(\mathfrak{q}) \leqq C_{\phi}\left(1+|\mathfrak{q}|^{3}\right) \text {, } \\
& \phi(\mathrm{E}, m) \geqq \varepsilon|m|^{2} \text {; } \\
& \exists C_{\mathscr{K}_{0}}, C_{\mathscr{K}} \in \mathbb{R}, \kappa_{0}, \varepsilon>0 \forall(\mathfrak{q}, \vartheta) \in \mathbb{R}_{\mathrm{sym}}^{3 \times 3} \times \mathbb{R}^{3} \times \mathbb{R}, \xi \in \mathbb{R}^{3}: \\
& \mathscr{K}_{0}(\mathfrak{q}, \vartheta) \xi \cdot \xi \geqq \kappa_{0}|\xi|^{2}, \\
& \left|\mathscr{K}_{0}(\mathfrak{q}, \vartheta)\right| \leqq C_{\mathscr{K}_{0}}\left(1+|\mathfrak{q}|^{6 / 5-\varepsilon}+|\vartheta|^{1 / 3-\varepsilon}\right) \text {, } \\
& |\mathscr{K}(\mathfrak{q}, \vartheta)| \leqq C_{\mathscr{K}} \sqrt{1+|\vartheta|} \text {; } \\
& \exists C_{\mathscr{A}}<\infty \forall(\mathfrak{q}, \vartheta) \in \mathbb{R}_{\mathrm{sym}}^{3 \times 3} \times \mathbb{R}^{3} \times \mathbb{R}: \quad|\mathscr{A}(\mathfrak{q}, \vartheta)| \leqq C_{\mathscr{A}} \sqrt{1+\phi(\mathfrak{q})+\vartheta} ; \\
& \zeta: \mathbb{R}^{L} \rightarrow \mathbb{R}^{+} \text {convex and positively homogeneous of degree } 1 \text {; } \\
& \chi: \mathbb{R}_{\mathrm{sym}}^{3 \times 3} \times \mathbb{R} \rightarrow \mathbb{R}^{L} \text { is bounded with } \chi^{\prime} \text { continuous and bounded; } \\
& \gamma: \mathbb{R} \rightarrow \mathbb{R}^{+} \text {continuous, } \exists \varepsilon>0 \forall \xi \in \mathbb{R}^{+}: \gamma(\xi) \geqq \varepsilon \xi ; \\
& \varrho>0, \quad \mathbb{D}, \mathbb{D}_{\mathrm{hpr}}, \mathbb{C}, \mathbb{C}_{\mathrm{hpr}} \text { symmetric positive definite; } \\
& \mathscr{S}: \mathbb{R}_{\mathrm{sym}}^{3 \times 3} \times \mathbb{R}^{3} \rightarrow \mathbb{R}_{\mathrm{sym}}^{3 \times 3} \\
& \text { continuous, bounded, (uniformly) positive definite; } \\
& f_{0} \in L^{2}\left(I ; L^{6 / 5}\left(\Omega ; \mathbb{R}^{3}\right)\right), \quad g_{0} \in L^{2}\left(I ; L^{4 / 3}\left(\Gamma ; \mathbb{R}^{3}\right)\right) ; \\
& u_{0} \in W^{2,2}\left(\Omega ; \mathbb{R}^{3}\right), \quad v_{0} \in L^{2}\left(\Omega ; \mathbb{R}^{3}\right) \text {; } \\
& m_{0} \in W^{1,2}\left(\Omega ; \mathbb{R}^{3}\right), \quad h_{0} \in L_{\text {curl }}^{2}\left(\Omega ; \mathbb{R}^{3}\right) \text {; } \\
& \theta_{0} \geqq 0, \quad q_{\mathrm{b}} \geqq 0, \quad \hat{c}\left(\mathrm{E}\left(u_{0}\right), m_{0}, \theta_{0}\right) \in L^{1}(\Omega), \quad q_{\mathrm{b}} \in L^{1}(\Sigma) ; \\
& h_{\mathrm{b}} \in L^{\infty}\left(I ; W^{1, \infty}\left(\Omega ; \mathbb{R}^{3}\right)\right) \cap W^{1,1}\left(I ; L^{2}\left(\Omega ; \mathbb{R}^{3}\right)\right) \text {. }
\end{aligned}
$$

In fact, we will need (72) only for $\vartheta \geqq 0$ in what follows. Note that (72f) ensures integrability of $\mathscr{K}_{0}(\mathfrak{q}, \vartheta) \nabla \vartheta$ if $\mathfrak{q} \in L^{6}\left(Q ; \mathbb{R}_{\text {sym }}^{3 \times 3} \times \mathbb{R}^{3}\right), \vartheta \in L^{5 / 3-\varepsilon}(Q)$, and $\nabla \vartheta \in L^{5 / 4-\varepsilon}\left(Q ; \mathbb{R}^{3}\right)$. Note also that the additional assumptions $(72 \mathrm{c})$ ensure that $\sigma_{m}(\mathfrak{q}, \vartheta)$ is in duality with $\dot{m}$, see (126) and (127) below.

In view of the definitions (62f-g), (71), and (72a), we can write (48) as

$$
\varphi_{\mathfrak{q}}^{\prime}(\mathfrak{q}, \mathscr{T}(\mathfrak{q}, \vartheta))=\phi^{\prime}(\mathfrak{q})+\mathscr{A}(\mathfrak{q}, \vartheta)
$$


that is,

$$
\begin{aligned}
& \sigma_{\mathrm{E}}(\mathfrak{q}, \vartheta)=\phi_{\mathrm{E}}^{\prime}(\mathfrak{q})+\mathscr{A}_{1}(\mathfrak{q}, \vartheta), \\
& \sigma_{m}(\mathfrak{q}, \vartheta)=\phi_{m}^{\prime}(\mathfrak{q})+\mathscr{A}_{2}(\mathfrak{q}, \vartheta) .
\end{aligned}
$$

The symmetry in (721) means that $\mathbb{D}: \mathbb{R}_{\text {sym }}^{d \times d} \rightarrow \mathbb{R}_{\text {sym }}^{d \times d}$, = that is, $\mathbb{D}_{i j k l}=\mathbb{D}_{j i k l}$, and similarly $\left[\mathbb{D}_{\mathrm{hpr}}\right]_{i j k l m n}=\left[\mathbb{D}_{\mathrm{hpr}}\right]_{i k j l m n}$, while for $\mathbb{H}_{\text {hyper }}$ we need $\left[\mathbb{C}_{\mathrm{hpr}}\right]_{i j k l m n}=$ $\left[\mathbb{C}_{\mathrm{hpr}}\right]_{\text {ikjlmn }}=\left[\mathbb{C}_{\mathrm{hpr}}\right]_{\text {lmnijk }}$,

The following main result is a consequence of Lemma 1 together with Proposition 1 below when taking into account that, due to the qualification (72o) of $u_{0}$, the regularization $u_{0 \tau}$, with all properties required in Proposition 1 , always exists.

Theorem 1. (Existence of a weak solution.) Let (72) hold. Then the transformed system (64) with the initial/boundary conditions (31b-d,f,g) and (65) possesses a weak solution $(u, m, \vartheta, h, e, \omega)$ according to the Definition 1 such that, in addition,

$$
\begin{aligned}
& \ddot{u} \in L^{2}\left(I ; W^{2,2}\left(\Omega ; \mathbb{R}^{3}\right)^{*}\right), \\
& \dot{\vartheta} \in L^{1}\left(I ; W^{3,2}(\Omega)^{*}\right), \\
& \dot{h} \in L^{2}\left(I ; L_{\text {curl }, 0}^{2}\left(\Omega ; \mathbb{R}^{3}\right)^{*}\right),
\end{aligned}
$$

and the total energy balance (46) with (51), as well as the electro-magnetomechanical energy balance (49) integrated over I, hold.

\section{Proof of Theorem 1}

We will prove Theorem 1 by several carefully assembled steps, merging and modifying various techniques from [35,39,40], using [38] for particular subsystems.

(A) We modify the problem (64) by a suitable extension for $\vartheta<0$ and then we build a semi-implicit time-discrete approximation of the modified problem. We use the equi-distant partition with the timestep $\tau>0$. We still use some auxiliary regularization by higher-order monotone terms of $\eta$-growth to compensate for the superlinear growth of the heat-source terms in (64c) and, for $\eta$ large enough, to ensure coercivity at each particular time step and to put all non-monotone terms in the position of lower-order terms. For $\eta$ large enough, we prove existence of a solution to (77) by the theory of set-valued pseudomonotone coercive operators with the set-valued part having a convex potential.

(B) We show non-negativity of the approximated temperature $\vartheta_{\tau}^{k} \geqq 0$.

(C) We perform the physically relevant a priori estimates, that is "real" energy bounds, see (102a-f).

(D) Using the $L^{1}$-theory for the heat equation together with a GagliardoNirenberg interpolation made simultaneously for the thermal and the magnetomechanical parts, we derive estimate on the temperature gradient, see $(102 \mathrm{~g})$.

(E) We let $\tau \rightarrow 0$ and we show, in particular, strong convergence of all quantities involved in dissipation $\xi$ from (64c); it is important to do these simultaneously 
to benefit from cancellation, especially of the magnetostrictive terms, see (136). Intimately related, we use (and also prove) energy conservation for which rates of $u, m$, and $h$ have to be proved sufficiently regular.

The steps (A)-(B) are executed in Lemma 1, while (C)-(D) are executed in Lemma 2, and eventually (E) is executed in Lemma 3 and Proposition 1.

In our proofs, positive constants dependent only on the data will be denoted by $C$. When two or more constants appear in the same formula, they will be denoted by a progressive integer subscript $\left(C_{1}, C_{2}\right.$, etc.).

To perform Step (A), we define

$$
f_{\tau}^{k}:=\frac{1}{\tau} \int_{(k-1) \tau}^{k \tau} g_{0}(t, \cdot) \mathrm{d} t, \quad j_{\mathrm{b}, \tau}^{k}:=\frac{1}{\tau} \int_{(k-1) \tau}^{k \tau} j_{\mathrm{b}}(t, \cdot) \mathrm{d} t,
$$

and similarly $h_{\mathrm{b}, \tau}^{k}, g_{\tau}^{k}$ and $q_{\mathrm{b}, \tau}^{k}$. It is important that this construction, based on a linear operator, inherits (50), that is, $n \times\left. h_{\mathrm{b}, \tau}^{k}\right|_{\Gamma}=j_{\mathrm{b}, \tau}^{k}$; in fact, $h_{\mathrm{b}, \tau}^{k}$ is used later only in (83) and in the proof of Lemma 2, but not in (77)-(79). Then we use the recursive formula for the six-tuple $\left(u_{\tau}^{k}, m_{\tau}^{k}, \vartheta_{\tau}^{k}, e_{\tau}^{k}, h_{\tau}^{k}, \omega_{\tau}^{k}\right)$ solving the system

$$
\begin{aligned}
& \varrho \frac{u_{\tau}^{k}-2 u_{\tau}^{k-1}+u_{\tau}^{k-2}}{\tau^{2}}-\operatorname{div}\left(\mathbf{S}_{\tau}^{k}-\operatorname{div} \mathbf{H}_{\tau}^{k}\right)=f_{\tau}^{k}-\mu_{0}\left(\nabla h_{\tau}^{k}\right)^{\top} m_{\tau}^{k} \quad \text { with } \\
& \mathrm{S}_{\tau}^{k}:=\sigma_{\mathrm{E}}\left(\mathfrak{q}_{\tau}^{k}, \vartheta_{\tau}^{k}\right)+\mathbb{D} \mathrm{E}\left(\frac{u_{\tau}^{k}-u_{\tau}^{k-1}}{\tau}\right)+\chi_{\mathrm{E}}^{\prime}\left(\mathfrak{q}_{\tau}^{k-1}\right)^{\top} \omega_{\tau}^{k}+\tau\left|\mathrm{E}\left(u_{\tau}^{k}\right)\right|^{\eta-2} \mathrm{E}\left(u_{\tau}^{k}\right) \text {, and } \\
& \mathbf{H}_{\tau}^{k}:=\mathbb{D}_{\mathrm{hpr}} \nabla \mathrm{E}\left(\frac{u_{\tau}^{k}-u_{\tau}^{k-1}}{\tau}\right)+\mathbb{C}_{\mathrm{hpr}} \nabla \mathrm{E}\left(u_{\tau}^{k}\right)+\tau\left|\nabla \mathrm{E}\left(u_{\tau}^{k}\right)\right|^{\eta-2} \nabla \mathrm{E}\left(u_{\tau}^{k}\right), \\
& \alpha \frac{m_{\tau}^{k}-m_{\tau}^{k-1}}{\tau}-\frac{m_{\tau}^{k}}{\gamma\left(\left|m_{\tau}^{k}\right|\right)} \times \frac{m_{\tau}^{k}-m_{\tau}^{k-1}}{\tau}-\lambda \Delta m_{\tau}^{k}+\sigma_{m}\left(\mathfrak{q}_{\tau}^{k}, \vartheta_{\tau}^{k}\right) \\
& \left.+\chi_{m}^{\prime}\left(\mathfrak{q}_{\tau}^{k-1}\right)^{\top} \omega_{\tau}^{k}-\mu_{0} h_{\tau}^{k}+\tau\left|m_{\tau}^{k}\right|^{\eta-2} m_{\tau}^{k}=\tau \operatorname{div}\left(\left|\nabla m_{\tau}^{k}\right|^{\eta-2}\right) \nabla m_{\tau}^{k}\right), \\
& \frac{\vartheta_{\tau}^{k}-\vartheta_{\tau}^{k-1}}{\tau}-\operatorname{div}\left(\mathscr{K}_{0}\left(\mathfrak{q}_{\tau}^{k}, \vartheta_{\tau}^{k}\right) \nabla \vartheta_{\tau}^{k}+\mathscr{K}\left(\mathfrak{q}_{\tau}^{k}, \vartheta_{\tau}^{k}\right) \vdots \nabla \mathfrak{q}_{\tau}^{k}\right)=\zeta\left(\chi^{\prime}\left(\mathfrak{q}_{\tau}^{k-1}\right) \frac{\mathfrak{q}_{\tau}^{k}-\mathfrak{q}_{\tau}^{k-1}}{\tau}\right) \\
& +\mathscr{S}\left(\mathfrak{q}_{\tau}^{k}, \vartheta_{\tau}^{k}\right) e_{\tau}^{k}: e_{\tau}^{k}+\left(1-\frac{\sqrt{\tau}}{2}\right) \operatorname{DE}\left(\frac{u_{\tau}^{k}-u_{\tau}^{k-1}}{\tau}\right): \mathrm{E}\left(\frac{u_{\tau}^{k}-u_{\tau}^{k-1}}{\tau}\right) \\
& +\mathbb{D}_{\mathrm{hpr}} \nabla \mathrm{E}\left(\frac{u_{\tau}^{k}-u_{\tau}^{k-1}}{\tau}\right) \vdots \nabla \mathrm{E}\left(\frac{u_{\tau}^{k}-u_{\tau}^{k-1}}{\tau}\right) \\
& +\left(1-\frac{\sqrt{\tau}}{2}\right) \alpha\left|\frac{m_{\tau}^{k}-m_{\tau}^{k-1}}{\tau}\right|^{2}+\mathscr{A}\left(\mathfrak{q}_{\tau}^{k}, \vartheta_{\tau}^{k}\right): \frac{\mathfrak{q}_{\tau}^{k}-\mathfrak{q}_{\tau}^{k-1}}{\tau}, \\
& \frac{h_{\tau}^{k}-h_{\tau}^{k-1}}{\tau}+\frac{\operatorname{curl} e_{\tau}^{k}}{\mu_{0}}=\nabla m_{\tau}^{k} \frac{u_{\tau}^{k}-u_{\tau}^{k-1}}{\tau}-\frac{m_{\tau}^{k}-m_{\tau}^{k-1}}{\tau}+\operatorname{div} \frac{u_{\tau}^{k}-u_{\tau}^{k-1}}{\tau} m_{\tau}^{k} \text {, } \\
& \operatorname{curl} h_{\tau}^{k}-\mathscr{S}\left(\mathfrak{q}_{\tau}^{k}, \vartheta_{\tau}^{k}\right) e_{\tau}^{k}=\tau\left|e_{\tau}^{k}\right|^{\eta-2} e_{\tau}^{k} \text {, } \\
& \omega_{\tau}^{k} \in \partial \zeta\left(\chi^{\prime}\left(\mathfrak{q}_{\tau}^{k-1}\right)\left(\mathfrak{q}_{\tau}^{k}-\mathfrak{q}_{\tau}^{k-1}\right)\right)
\end{aligned}
$$

for $k=1, \ldots, K_{\tau}:=T / \tau$, starting from $k=1$ by using

$$
u_{\tau}^{0}=u_{0, \tau}, u_{\tau}^{-1}=u_{0, \tau}-\tau v_{0}, m_{\tau}^{0}=m_{0, \tau}, \vartheta_{\tau}^{0}=\hat{c}\left(\mathrm{E}\left(u_{0}\right), m_{0}, \theta_{0}\right), h_{\tau}^{0}=h_{0},
$$

completed with the corresponding regularized boundary conditions. 


$$
\begin{aligned}
& \left(\sigma_{\mathrm{E}}\left(\mathfrak{q}_{\tau}^{k}, \vartheta_{\tau}^{k}\right)+\mathbb{D} \mathrm{E}\left(\frac{u_{\tau}^{k}-u_{\tau}^{k-1}}{\tau}\right)+\chi_{\mathrm{E}}^{\prime}\left(\mathfrak{q}_{\tau}^{k}\right)^{\top} \omega_{\tau}^{k}+\tau\left|\mathrm{E}\left(u_{\tau}^{k}\right)\right|^{\eta-2} \mathrm{E}\left(u_{\tau}^{k}\right)\right. \\
& \left.-\operatorname{div} \mathbf{H}_{\tau}^{k}\right) \cdot v-\operatorname{div}^{\mathrm{s}}\left(\mathbf{H}_{\tau}^{k} \cdot v\right)-\mu_{0}\left(h_{\tau}^{k} \cdot m_{\tau}^{k}\right) v=g_{\tau}^{k}, \\
& \mathbf{H}_{\tau}^{k}:(n \otimes n)=0 \quad \text { with } \\
& \mathbf{H}_{\tau}^{k}=\left(\mathbb{C}_{\mathrm{hpr}}+\tau \mathbb{I}\left|\nabla \mathrm{E}\left(u_{\tau}^{k}\right)\right|^{\eta-2}\right) \nabla \mathrm{E}\left(u_{\tau}^{k}\right)+\mathbb{D}_{\mathrm{hpr}} \nabla \mathrm{E}\left(\frac{u_{\tau}^{k}-u_{\tau}^{k-1}}{\tau}\right), \\
& \left(1+\tau\left|\nabla m_{\tau}^{k}\right|^{\eta-2} \nabla m_{\tau}^{k}\right) \frac{\partial m_{\tau}^{k}}{\partial n}=0 \\
& \mathscr{K}_{0}\left(\mathfrak{q}_{\tau}^{k}, \vartheta_{\tau}^{k}\right) \frac{\partial \vartheta}{\partial n}+\mathscr{K}_{1}\left(\mathfrak{q}_{\tau}^{k}, \vartheta_{\tau}^{k}\right) \frac{\partial \mathrm{E}\left(u_{\tau}^{k}\right)}{\partial n}+\mathscr{K}_{2}\left(\mathfrak{q}_{\tau}^{k}, \vartheta_{\tau}^{k}\right) \frac{\partial m_{\tau}^{k}}{\partial n}=q_{\mathrm{b}, \tau}^{k}, \\
& n \times h_{\tau}^{k}=j_{\mathrm{b}, \tau}^{k} .
\end{aligned}
$$

Note that in (78) we use regularized initial conditions for those quantities which involve the $\eta$-regularizing terms yielding $L^{\infty}$-estimates in time. For this, we assume

$$
\begin{aligned}
& u_{0, \tau} \in W^{2, \eta}\left(\Omega ; \mathbb{R}^{3}\right), \quad\left\|u_{0, \tau}\right\|_{W^{2, \eta}\left(\Omega ; \mathbb{R}^{3}\right)} \\
& =o\left(\tau^{-1 / \eta}\right), \quad u_{0, \tau} \rightarrow u_{0} \text { in } W^{2,2}\left(\Omega ; \mathbb{R}^{3}\right), \\
& m_{0, \tau} \in W^{1, \eta}\left(\Omega ; \mathbb{R}^{3}\right), \quad\left\|m_{0, \tau}\right\|_{W^{1, \eta}\left(\Omega ; \mathbb{R}^{3}\right)} \\
& =o\left(\tau^{-1 / \eta}\right), \quad m_{0, \tau} \rightarrow m_{0} \text { in } W^{1,2}\left(\Omega ; \mathbb{R}^{3}\right) .
\end{aligned}
$$

Lemma 1. (Existence of solutions to (77).) Let (72) and (80) hold, and let the regularization exponent $\eta$ be sufficiently large, namely $\eta>8$. Then the recursive boundary-value problem (77)-(79) has a weak solution

$$
\begin{aligned}
u_{\tau}^{k} & \in W^{2, \eta}\left(\Omega ; \mathbb{R}^{3}\right), \\
m_{\tau}^{k} & \in W^{1, \eta}\left(\Omega ; \mathbb{R}^{3}\right), \\
\vartheta_{\tau}^{k} & \in W^{1,2}(\Omega), \\
h_{\tau}^{k} & \in L_{\text {curl }}^{2, \eta^{\prime}}\left(\Omega ; \mathbb{R}^{3}\right), \\
e_{\tau}^{k} & \in L_{\text {curl }}^{\eta, 2}\left(\Omega ; \mathbb{R}^{3}\right), \\
\omega_{\tau}^{k} & \in L^{\infty}\left(\Omega ; \mathbb{R}^{L}\right),
\end{aligned}
$$

such that $\vartheta_{\tau}^{k} \geqq 0$ for any $k=1, \ldots, T / \tau$.

Proof. We apply the abstract existence theorem for underlying set-valued nonlinear pseudomonotone coercive operators sending the six-tuple $(u, m, \vartheta, h, e)$ from the reflexive Banach space involved in (81a-e) to its dual.

To prove the coercivity of this operator resulting from (77)-(79), the particular equations $(77 \mathrm{a}-\mathrm{e})$ in the weak formulation that also involve the respective boundary conditions (79) have to be tested, respectively, by $\tau^{-1} u_{\tau}^{k}, \tau^{-1} m_{\tau}^{k}, \vartheta_{\tau}^{k}, h_{\tau}^{k}$, and $e_{\tau}^{k}$. f inequalities. Let us briefly discuss the estimation of the non-monotone terms under this test. On setting $\sigma_{\mathfrak{q}}=\left(\sigma_{\mathrm{E}}, \sigma_{m}\right)$, and abbreviating $\mathrm{E}_{\tau}^{k}:=\mathrm{E}\left(u_{\tau}^{k}\right)$, we have by (72b) and (72h) 


$$
\begin{aligned}
\left|\int_{\Omega} \sigma_{\mathfrak{q}}\left(\mathfrak{q}_{\tau}^{k}, \vartheta_{\tau}^{k}\right) \cdot \mathfrak{q}_{\tau}^{k} \mathrm{~d} x\right| \leqq & \left|\int_{\Omega} \phi_{\mathfrak{q}}^{\prime}\left(\mathfrak{q}_{\tau}^{k}\right) \cdot \mathfrak{q}_{\tau}^{k} \mathrm{~d} x\right|+\left|\int_{\Omega} \mathscr{A}\left(\mathfrak{q}_{\tau}^{k}, \vartheta_{\tau}^{k}\right) \cdot \mathfrak{q}_{\tau}^{k} \mathrm{~d} x\right| \\
\leqq & C+C \int_{\Omega}\left|\mathfrak{q}_{\tau}^{k}\right|^{6-\varepsilon} \mathrm{d} x+C \int_{\Omega} \sqrt{\vartheta_{\tau}^{k}}\left|\mathfrak{q}_{\tau}^{k}\right| \mathrm{d} x \\
\leqq & C+\delta\left\|\mathrm{E}_{\tau}^{k}\right\|_{L^{\eta}\left(\Omega ; \mathbb{R}_{\mathrm{sym}}^{3 \times 3}\right)}^{\eta} \\
& +\delta\left\|m_{\tau}^{k}\right\|_{L^{\eta}\left(\Omega ; \mathbb{R}^{3}\right)}^{\eta}+\delta\left\|\vartheta_{\tau}^{k}\right\|_{L^{2}(\Omega)}^{2}
\end{aligned}
$$

where the last inequality requires $\eta \geqq 6$.

The gyroscopic term vanishes, that is, $\frac{m_{\tau}^{k}}{\gamma\left(\left|m_{\tau}^{k}\right|\right)} \times \frac{m_{\tau}^{k}-m_{\tau}^{k-1}}{\tau} \cdot m_{\tau}^{k}=0$, due to the orthogonality of $m_{\tau}^{k} \times\left(m_{\tau}^{k}-m_{\tau}^{k-1}\right)$ and $m_{\tau}^{k}$.

The mentioned test of the Maxwell equations (77d) by $h_{\tau}^{k}$ and (77e) by $e_{\tau}^{k}$ uses the "curl-cancellation" property (7) with $h=h_{\tau}^{k} \in L_{\text {curl }}^{2, \eta^{\prime}}\left(\Omega ; \mathbb{R}^{3}\right)$ and $e=e_{\tau}^{k} \in$ $L_{\text {curl }}^{\eta, 2}\left(\Omega ; \mathbb{R}^{3}\right)$, with the arising boundary term also counting $(79 \mathrm{e})$, and then uses the estimate

$$
\begin{aligned}
\int_{\Gamma}\left(n \times h_{\tau}^{k}\right) \cdot e_{\tau}^{k} \mathrm{~d} S & =\int_{\Gamma} j_{\mathrm{b}, \tau}^{k} \cdot e_{\tau}^{k} \mathrm{~d} S=\int_{\Omega} e_{\tau}^{k} \cdot \operatorname{curl} h_{\mathrm{b}, \tau}^{k}-h_{\mathrm{b}, \tau}^{k} \cdot \operatorname{curl} e_{\tau}^{k} \mathrm{~d} x \\
& \leqq 2\left\|h_{\mathrm{b}, \tau}^{k}\right\|_{L_{\text {curl }}^{2}\left(\Omega ; \mathbb{R}^{3}\right)}\left\|e_{\tau}^{k}\right\|_{L_{\text {curl }}^{2}\left(\Omega ; \mathbb{R}^{3}\right)} \\
& \leqq C_{\eta}\left\|h_{\mathrm{b}, \tau}^{k}\right\|_{L_{\text {curl }}^{2}\left(\Omega ; \mathbb{R}^{3}\right)}\left\|e_{\tau}^{k}\right\|_{L_{\text {curl }}^{\eta, 2}\left(\Omega ; \mathbb{R}^{3}\right)} \cdot
\end{aligned}
$$

Next, we use the cancellation of $\pm \tau^{-1} m_{\tau}^{k} \cdot h_{\tau}^{k}$ in (77b) and (77d), and the cancellation (35) of the magnetostrictive terms in (77a) and (77d), that is,

$$
\begin{aligned}
& \int_{\Omega}\left(\left(\operatorname{div} u_{\tau}^{k}\right) m_{\tau}^{k}+\nabla m_{\tau}^{k} u_{\tau}^{k}\right) \cdot h_{\tau}^{k} \mathrm{~d} x \\
& \quad=\int_{\Gamma}\left(m_{\tau}^{k} \cdot h_{\tau}^{k}\right)\left(u_{\tau}^{k} \cdot n\right) \mathrm{d} S-\int_{\Omega}\left(\nabla h_{\tau}^{k}\right)^{\top} m_{\tau}^{k} \cdot u_{\tau}^{k} \mathrm{~d} x,
\end{aligned}
$$

see also (35), while the remaining right-hand side terms in (77d) can be estimated as

$$
\begin{aligned}
& \left|\int_{\Omega}\left(\nabla m_{\tau}^{k} u_{\tau}^{k-1}+\left(\operatorname{div} u_{\tau}^{k-1}\right) m_{\tau}^{k}\right) \cdot h_{\tau}^{k} \mathrm{~d} x\right| \\
& \quad \leqq C_{\delta}\left\|u_{\tau}^{k-1}\right\|_{L^{\infty}\left(\Omega ; \mathbb{R}^{3}\right)}^{2 \eta /(2 \eta-2-\eta)}+\delta\left\|\nabla m_{\tau}^{k}\right\|_{W^{1, \eta}\left(\Omega ; \mathbb{R}^{3}\right)}^{\eta}+\delta\left\|h_{\tau}^{k}\right\|_{L^{2}\left(\Omega ; \mathbb{R}^{2}\right)}^{2},
\end{aligned}
$$

and then absorbed in the left-hand side, provided $\eta>2$.

Altogether, counting the positive-definiteness assumptions (72b,d,e) and the regularizing terms of $\eta$-growth in $(77 \mathrm{a}, \mathrm{b}, \mathrm{e})$, the coercive left-hand side terms are of the type

$$
\begin{aligned}
& \left\|u_{\tau}^{k}\right\|_{L^{2}\left(\Omega ; \mathbb{R}^{3}\right)}^{2}+\left\|\mathrm{E}_{\tau}^{k}\right\|_{W^{1, \eta}\left(\Omega ; \mathbb{R}_{\text {sym }}^{3 \times 3}\right)}^{\eta}+\left\|m_{\tau}^{k}\right\|_{W^{1, \eta}\left(\Omega ; \mathbb{R}^{3}\right)}^{\eta} \\
& +\left\|\vartheta_{\tau}^{k}\right\|_{W^{1,2}(\Omega)}^{2}+\left\|h_{\tau}^{k}\right\|_{L^{2}\left(\Omega ; \mathbb{R}^{3}\right)}^{2}+\left\|e_{\tau}^{k}\right\|_{L^{\eta}\left(\Omega ; \mathbb{R}^{3}\right)}^{\eta} .
\end{aligned}
$$


This dominates the growth of the remaining non-monotone terms on the right-hand side of the heat equation (77c). Moreover, from (77d) we can see that

$$
\begin{aligned}
& \left\|\operatorname{curl} e_{\tau}^{k}\right\|_{L^{2}\left(\Omega ; \mathbb{R}^{3}\right)} \\
& \quad=\mu_{0}\left\|\nabla m_{\tau}^{k} \frac{u_{\tau}^{k}-u_{\tau}^{k-1}}{\tau}+\operatorname{div} \frac{u_{\tau}^{k}-u_{\tau}^{k-1}}{\tau} m_{\tau}^{k}-\frac{m_{\tau}^{k}-m_{\tau}^{k-1}}{\tau}-\frac{h_{\tau}^{k}-h_{\tau}^{k-1}}{\tau}\right\|_{L^{2}\left(\Omega ; \mathbb{R}^{3}\right)} \\
& \quad \leqq C\left(1+\left\|u_{\tau}^{k}\right\|_{W^{1,2}\left(\Omega ; \mathbb{R}^{3}\right)}^{2}+\left\|m_{\tau}^{k}\right\|_{W^{1,2}\left(\Omega ; \mathbb{R}^{3}\right)}^{2}+\left\|h_{\tau}^{k}\right\|_{L^{2}\left(\Omega ; \mathbb{R}^{3}\right)}\right)
\end{aligned}
$$

while from (77e), for $\eta \geqq 2$, we can also see that

$$
\begin{aligned}
\left\|\operatorname{curl} h_{\tau}^{k}\right\|_{L^{\eta^{\prime}}\left(\Omega ; \mathbb{R}^{3}\right)} & =\left\|\tau\left|e_{\tau}^{k}\right|^{\eta-2} e_{\tau}^{k}+\mathscr{S}\left(\mathfrak{q}_{\tau}^{k}, \vartheta_{\tau}^{k}\right) e_{\tau}^{k}\right\|_{L^{\eta^{\prime}}\left(\Omega ; \mathbb{R}^{3}\right)} \\
& \leqq \sup |\mathscr{S}(\cdot, \cdot)|\left\|e_{\tau}^{k}\right\|_{L^{\eta^{\prime}}\left(\Omega ; \mathbb{R}^{3}\right)}+\tau\left\|e_{\tau}^{k}\right\|_{L^{\eta}\left(\Omega ; \mathbb{R}^{3}\right)}^{\eta-1},
\end{aligned}
$$

so that we also control both $\left\|\operatorname{curl} e_{\tau}^{k}\right\|_{L^{2}\left(\Omega ; \mathbb{R}^{3}\right)}$ and $\left\|\operatorname{curl} h_{\tau}^{k}\right\|_{L^{\eta^{\prime}}\left(\Omega ; \mathbb{R}^{3}\right)}$, and thus we obtain coercivity on the spaces indicated in $(81 \mathrm{~d}, \mathrm{e})$, as well as ensuring validity of the curl-cancellation formula (7).

Further, one can estimate the $\mathscr{K}$-term by using Hölder's inequality for three factors with exponents $4, \eta$, and 2 and by using $(72 \mathrm{~g})$

$$
\begin{aligned}
& \left|\int_{\Omega} \mathscr{K}\left(\mathfrak{q}_{\tau}^{k}, \vartheta_{\tau}^{k}\right) \nabla \mathfrak{q}_{\tau}^{k} \cdot \nabla \vartheta_{\tau}^{k} \mathrm{~d} x\right| \\
& \quad \leqq \int_{\Omega} C_{\mathscr{K}} \sqrt{1+\left|\vartheta_{\tau}^{k}\right|}\left|\nabla \mathfrak{q}_{\tau}^{k}\right|\left|\nabla \vartheta_{\tau}^{k}\right| \mathrm{d} x \leqq \int_{\Omega} C_{\mathscr{K}}\left(1+\sqrt{\left|\vartheta_{\tau}^{k}\right|}\right)\left|\nabla \mathfrak{q}_{\tau}^{k}\right|\left|\nabla \vartheta_{\tau}^{k}\right| \mathrm{d} x \\
& \quad \leqq C_{\delta, \eta}+\delta\left\|\vartheta_{\tau}^{k}\right\|_{L^{2}(\Omega)}^{2}+\delta\left\|\nabla \mathrm{E}_{\tau}^{k}\right\|_{L^{\eta}\left(\Omega ; \mathbb{R}^{3 \times 3 \times 3}\right)}^{\eta}+\delta\left\|\mathrm{E}_{\tau}^{k}\right\|_{L^{\eta}\left(\Omega ; \mathbb{R}_{\mathrm{sym}}\right)}^{\eta \times 3}+\delta\left\|\nabla \vartheta_{\tau}^{k}\right\|_{L^{2}\left(\Omega ; \mathbb{R}^{d}\right)}^{2},
\end{aligned}
$$

with $\delta>0$ arbitrarily small and some $C_{\delta, \eta}$ depending on $\eta$ and $\delta$; here we needed that $\eta>4$. The heat sources with quadratic growth can be estimated as

$$
\begin{aligned}
& \left|\int_{\Omega}\left(\mathbb{D}_{\tau}^{k}: \mathrm{E}_{\tau}^{k}+\mathbb{D}_{\mathrm{hpr}} \nabla \mathrm{E}_{\tau}^{k}: \nabla \mathrm{E}_{\tau}^{k}\right) \vartheta_{\tau}^{k} \mathrm{~d} x\right| \\
& \quad \leqq C_{\delta, \eta}+\delta\left\|\mathrm{E}_{\tau}^{k}\right\|_{L^{\eta}\left(\Omega ; \mathbb{R}_{\mathrm{sym}}^{3 \times 3}\right)}^{\eta}+\delta\left\|\nabla \mathrm{E}_{\tau}^{k}\right\|_{L^{\eta}\left(\Omega ; \mathbb{R}^{3 \times 3 \times 3}\right)}^{\eta}+\delta\left\|\vartheta_{\tau}^{k}\right\|_{L^{2}(\Omega)}^{2}, \\
& \left.\left|\int_{\Omega} \alpha\right| m_{\tau}^{k}\right|^{2} \vartheta_{\tau}^{k} \mathrm{~d} x \mid \leqq C_{\delta, \eta}+\delta\left\|m_{\tau}^{k}\right\|_{L^{\eta}\left(\Omega ; \mathbb{R}^{3}\right)}^{\eta}+\delta\left\|\vartheta_{\tau}^{k}\right\|_{L^{2}(\Omega)}^{2}, \quad \text { and } \\
& \left|\int_{\Omega}\left(\mathscr{S}\left(\mathfrak{q}_{\tau}^{k}, \vartheta_{\tau}^{k}\right) e_{\tau}^{k}: e_{\tau}^{k}\right) \vartheta_{\tau}^{k} \mathrm{~d} x\right| \leqq C_{\delta, \eta}+\delta\left\|e_{\tau}^{k}\right\|_{L^{\eta}\left(\Omega ; \mathbb{R}^{3}\right)}^{\eta}+\delta\left\|\vartheta_{\tau}^{k}\right\|_{L^{2}(\Omega)}^{2} .
\end{aligned}
$$

Here, again, we used that $\eta>4$. Also, because of the boundedness assumption (72j), we have the estimate

$$
\begin{aligned}
\int_{\Omega} \zeta\left(\chi^{\prime}\left(\mathfrak{q}_{\tau}^{k}, \vartheta_{\tau}^{k}\right): \frac{\mathfrak{q}_{\tau}^{k}-\mathfrak{q}_{\tau}^{k-1}}{\tau}\right) \mathrm{d} x & \leqq \int_{\Omega}\left|\chi^{\prime}\left(\mathfrak{q}_{\tau}^{k}, \vartheta_{\tau}^{k}\right) \| \frac{\mathfrak{q}_{\tau}^{k}-\mathfrak{q}_{\tau}^{k-1}}{\tau}\right| \mathrm{d} x \\
& \leqq C_{k, \tau, \eta}+\delta\left\|\mathrm{E}_{\tau}^{k}\right\|_{L^{\eta}\left(\Omega ; \mathbb{R}_{\mathrm{sym}}^{3 \times 3}\right)}^{\eta}+\delta\left\|m_{\tau}^{k}\right\|_{\left.L^{\eta}\left(\Omega ; \mathbb{R}^{3}\right)\right)}^{\eta} .
\end{aligned}
$$


By (72h), the $\mathscr{A}$-term in (77c) can be estimated as

$$
\begin{aligned}
& \left|\int_{\Omega} \mathcal{A}\left(\mathfrak{q}_{\tau}^{k}, \vartheta_{\tau}^{k}\right): \mathfrak{q}_{\tau}^{k} \vartheta_{\tau}^{k} \mathrm{~d} x\right| \\
& \quad \leqq C_{\mathcal{A}} \int_{\Omega} \sqrt{1+\phi\left(\mathfrak{q}_{\tau}^{k}\right)+\left|\vartheta_{\tau}^{k}\right|}\left|\mathfrak{q}_{\tau}^{k}\right|\left|\vartheta_{\tau}^{k}\right| \mathrm{d} x \\
& \quad \leqq C_{\mathcal{A}}\left(1+\int_{\Omega} \sqrt{\phi\left(\mathfrak{q}_{\tau}^{k}\right)}\left|\mathfrak{q}_{\tau}^{k}\right|\left|\vartheta_{\tau}^{k}\right| \mathrm{d} x+\int_{\Omega}\left|\vartheta_{\tau}^{k}\right|^{\frac{3}{2}}\left|\mathfrak{q}_{\tau}^{k}\right| \mathrm{d} x\right) \\
& \quad \leqq C_{\mathcal{A}}+C_{\mathcal{A}} C_{\phi} \int_{\Omega}\left|\mathfrak{q}_{\tau}^{k}\right|^{4}\left|\vartheta_{\tau}^{k}\right| \mathrm{d} x+C_{\mathcal{A}} \int_{\Omega}|\vartheta|^{\frac{3}{2}}\left|\mathfrak{q}_{\tau}^{k}\right| \mathrm{d} x \\
& \quad \leqq C_{\delta, \eta}+\delta\left\|m_{\tau}^{k}\right\|_{L^{\eta}\left(\Omega ; \mathbb{R}^{3}\right)}^{\eta}+\delta\left\|\mathrm{E}_{\tau}^{k}\right\|_{L^{\eta}\left(\Omega ; \mathbb{R}_{\mathrm{sym}}^{3 \times 3}\right)}^{\eta}+\delta\left\|\vartheta_{\tau}^{k}\right\|_{L^{2}(\Omega)}^{2},
\end{aligned}
$$

provided that $\eta>8$.

The mentioned test of $(77 \mathrm{f})$ in the weak formulation, that is,

$$
\zeta(v)-\omega_{\tau}^{k} \cdot\left(v-\chi^{\prime}\left(\mathfrak{q}_{\tau}^{k-1}\right)\left(\mathfrak{q}_{\tau}^{k}-\mathfrak{q}_{\tau}^{k-1}\right)\right) \geqq \zeta\left(\chi^{\prime}\left(\mathfrak{q}_{\tau}^{k-1}\right)\left(\mathfrak{q}_{\tau}^{k}-\mathfrak{q}_{\tau}^{k-1}\right)\right),
$$

by $v=0$ gives

$$
\begin{aligned}
\zeta\left(\chi^{\prime}\left(\mathfrak{q}_{\tau}^{k-1}\right)\left(\mathfrak{q}_{\tau}^{k}-\mathfrak{q}_{\tau}^{k-1}\right)\right) \leqq & \chi_{\mathrm{E}}^{\prime}\left(\mathfrak{q}_{\tau}^{k-1}\right) \omega_{\tau}^{k}: \mathrm{E}\left(u_{\tau}^{k}\right)+\chi_{m}\left(\mathfrak{q}_{\tau}^{k-1}\right) \omega_{\tau}^{k} \cdot m_{\tau}^{k} \\
& -\chi_{\mathrm{E}}^{\prime}\left(\mathfrak{q}_{\tau}^{k-1}\right) \omega_{\tau}^{k}: \mathrm{E}\left(u_{\tau}^{k-1}\right)-\chi_{m}^{\prime}\left(\mathfrak{q}_{\tau}^{k-1}\right) \omega_{\tau}^{k} \cdot m_{\tau}^{k-1},
\end{aligned}
$$

with the last two terms being bounded in $L^{6}(\Omega)$, since $\omega_{\tau}^{k} \in L^{\infty}\left(\Omega ; \mathbb{R}^{L}\right)$. The pseudomonotonicity of the full operator is obvious because all non-monotone terms are of a lower order, with the exception of the $\mathscr{K}$-term in $(77 \mathrm{c})$. This term is, however, linear in $\nabla \mathfrak{q}_{\tau}^{k}$, hence weakly continuous. Then the claimed existence follows standardly by the theory of pseudomonotone operators using the classical Brézis theorem [10] generalized for set-valued mappings having a convex potential, see for example [37, Sect. 5.3]; in fact, this is a very special case of the general set-valued pseudomonotone operators merging only standard concepts from convex analysis and single-valued monotone and compact mappings. Here it is important that we have made a semi-implicit discretisation so that the nonsmooth part has a potential $\mathfrak{q} \mapsto \zeta\left(\chi^{\prime}\left(\mathfrak{q}_{\tau}^{k-1}\right)\left(\mathfrak{q}-\mathfrak{q}_{\tau}^{k-1}\right)\right)$, which is indeed convex.

Eventually, one can test (77c) by $\left(\vartheta_{\tau}^{k}\right)^{-}$, which yields $\vartheta_{\tau}^{k} \geqq 0$. For this, we can realize that, for the moment, we can define $\mathscr{A}(\mathfrak{q}, \vartheta)=0$ for $\vartheta \leqq 0$, and then find that this possible re-definition is, in fact, irrelevant for this particular solution.

Let us define the piecewise affine interpolants $u_{\tau}$ and $\vartheta_{\tau}$ by

$$
u_{\tau}(t):=\frac{t-(k-1) \tau}{\tau} u_{\tau}^{k}+\frac{k \tau-t}{\tau} u_{\tau}^{k-1} \quad \text { for } t \in[(k-1) \tau, k \tau],
$$

with $k=0, \ldots, K_{\tau}:=T / \tau$. We also define the backward piecewise constant interpolant $\bar{u}_{\tau}$ and $\underline{u}_{\tau}$ by

$$
\begin{array}{ll}
\bar{u}_{\tau}(t):=u_{\tau}^{k}, & \text { for } t \in((k-1) \tau, k \tau], k=1, \ldots, K_{\tau}, \\
\underline{u}_{\tau}(t):=u_{\tau}^{k-1}, & \text { for } t \in[(k-1) \tau, k \tau), k=1, \ldots, K_{\tau} .
\end{array}
$$


Similarly, we define $m_{\tau}, \bar{m}_{\tau}, \underline{m}_{\tau}, \bar{\vartheta}_{\tau}, \vartheta_{\tau}, \bar{g}_{\tau}, \bar{h}_{\mathrm{b}, \tau}$, etc. We will also need the piecewise affine interpolant of the (piecewise constant) velocity $\frac{\partial u_{\tau}}{\partial t}$, which we denote by $\left[\frac{\partial u_{\tau}}{\partial t}\right]^{i}$, that is,

$$
\left[\dot{u}_{\tau}\right]^{\mathrm{i}}(t):=\frac{t-(k-1) \tau}{\tau} \dot{u}_{\tau}(k \tau)+\frac{k \tau-t}{\tau} \dot{u}_{\tau}(k \tau-\tau) \quad \text { for } t \in((k-1) \tau, k \tau] .
$$

Note that $\ddot{u}_{\tau}^{\mathrm{i}}:=\frac{\partial}{\partial t}\left[\dot{u}_{\tau}\right]^{\mathrm{i}}$ is piecewise constant with the values $\frac{u_{\tau}^{k}-2 u_{\tau}^{k-1}+u_{\tau}^{k-2}}{\tau^{2}}$ on the particular subintervals $((k-1) \tau, k \tau)$.

In terms of these interpolants, we can write the approximate system (77) in a more "condensed" form closer to the desired continuous system (64) as treated in the weak formulation (70):

$$
\begin{aligned}
& \varrho \ddot{u}_{\tau}^{\mathrm{i}}-\operatorname{div}\left(\sigma_{\mathrm{E}}\left(\overline{\mathfrak{q}}_{\tau}, \bar{\vartheta}_{\tau}\right)+\mathbb{D} \mathrm{E}\left(\dot{u}_{\tau}\right)+\chi_{\mathrm{E}}^{\prime}\left(\underline{\mathfrak{q}}_{\tau}\right)^{\top} \bar{\omega}_{\tau}+\tau\left|\mathrm{E}\left(\bar{u}_{\tau}\right)\right|^{\eta-2} \mathrm{E}\left(\bar{u}_{\tau}\right)\right. \\
& \left.-\operatorname{div}\left(\mathbb{C}_{\mathrm{hpr}} \nabla \mathrm{E}\left(\bar{u}_{\tau}\right)+\mathbb{D}_{\mathrm{hpr}} \nabla \mathrm{E}\left(\dot{u}_{\tau}\right)+\tau\left|\nabla \mathrm{E}\left(\bar{u}_{\tau}\right)\right|^{\eta-2} \nabla \mathrm{E}\left(\bar{u}_{\tau}\right)\right)\right) \\
& \quad=\bar{f}_{\tau}-\mu_{0}\left(\nabla \bar{h}_{\tau}\right)^{\top} \bar{m}_{\tau}, \\
& \alpha \dot{m}_{\tau}-\frac{\bar{m}_{\tau} \times \dot{m}_{\tau}}{\gamma\left(\left|\bar{m}_{\tau}\right|\right)}-\lambda \Delta \bar{m}_{\tau}+\sigma_{m}\left(\overline{\mathfrak{q}}_{\tau}, \bar{\vartheta}_{\tau}\right)+\chi_{m}^{\prime}\left(\underline{\mathfrak{q}}_{\tau}\right)^{\top} \bar{\omega}_{\tau} \\
& \quad-\mu_{0} \bar{h}_{\tau}+\tau\left|\bar{m}_{\tau}\right|^{\eta-2} \bar{m}_{\tau}=\tau \operatorname{div}\left(\left|\nabla \bar{m}_{\tau}\right|^{\eta-2} \nabla \bar{m}_{\tau}\right), \\
& \left.\left.\left.\quad+\dot{\vartheta}_{\tau}-\operatorname{div}\left(\mathscr{K}_{0}\left(\overline{\mathfrak{q}}_{\tau}, \bar{\vartheta}_{\tau}\right) \nabla \bar{\vartheta}_{\tau}+\overline{\mathfrak{q}}_{\tau}, \bar{\vartheta}_{\tau}\right) \bar{e}_{\tau} \cdot \bar{e}_{\tau}+\left(1-\frac{\sqrt{\mathfrak{q}}}{2}\right) \bar{\vartheta}_{\tau}\right) \vdots \nabla \overline{\mathfrak{q}}_{\tau}\right)=\zeta\left(\chi_{\mathrm{E}}^{\prime} \underline{\mathfrak{q}}_{\tau}\right) \mathrm{E}\left(\dot{u}_{\tau}\right)+\chi_{m}^{\prime}\left(\dot{q}_{\tau}\right): \mathrm{E}\left(\dot{u}_{\tau}\right)\right) \\
& \quad+\mathbb{D}_{\mathrm{hpr}} \nabla \mathrm{E}\left(\dot{u}_{\tau}\right) \vdots \nabla \mathrm{E}\left(\dot{u}_{\tau}\right)+\left(1-\frac{\sqrt{\tau}}{2}\right) \alpha\left|\dot{m}_{\tau}\right|^{2}+\mathscr{A}\left(\overline{\mathfrak{q}}_{\tau}, \bar{\vartheta}_{\tau}\right): \dot{\mathfrak{q}}_{\tau}, \\
& \dot{h}_{\tau}+\dot{m}_{\tau}+\frac{\operatorname{curl} \bar{e}_{\tau}}{\mu_{0}}=-\nabla \bar{m}_{\tau} \dot{u}_{\tau}-\left(\operatorname{div} \dot{u}_{\tau}\right) \bar{m}_{\tau}, \\
& \operatorname{curl} \bar{h}_{\tau}-\mathscr{S}\left(\overline{\mathfrak{q}}_{\tau}, \bar{\vartheta}_{\tau}\right) \bar{e}_{\tau}=\tau\left|\bar{e}_{\tau}\right|^{\eta-2} \bar{e}_{\tau}, \\
& \left.\bar{\omega}_{\tau} \in \partial \zeta\left(\chi_{\mathrm{E}}^{\prime}\left(\underline{\mathfrak{q}}_{\tau}\right) \mathrm{E}\left(\dot{u}_{\tau}\right)+\chi_{m}^{\prime}\left(\underline{\mathfrak{q}}_{\tau}\right) \dot{m}_{\tau}\right)\right)
\end{aligned}
$$

with the corresponding initial and boundary conditions.

Lemma 2. (A priori estimates.) Let the assumptions of Lemma 1 and (80) hold. Then, there exists $\tau_{0}>0$ such that, for sufficiently small $0<\tau \leqq \tau_{0}$, it holds:

$$
\begin{aligned}
& \left\|u_{\tau}\right\|_{W^{1, \infty}\left(I ; L^{2}\left(\Omega ; \mathbb{R}^{3}\right)\right) \cap W^{1,2}\left(I ; W^{2,2}\left(\Omega ; \mathbb{R}^{3}\right)\right)} \leqq C, \\
& \left\|m_{\tau}\right\|_{L^{\infty}\left(I ; W^{1,2}\left(\Omega ; \mathbb{R}^{3}\right)\right) \cap W^{1,2}\left(I ; L^{2}\left(\Omega ; \mathbb{R}^{3}\right)\right)} \leqq C, \\
& \left\|\bar{\vartheta}_{\tau}\right\|_{L^{\infty}\left(I ; L^{1}(\Omega)\right)} \leqq C, \\
& \left\|h_{\tau}\right\|_{L^{\infty}\left(I ; L^{2}\left(\Omega ; \mathbb{R}^{3}\right)\right)} \leqq C, \\
& \left\|\bar{e}_{\tau}\right\|_{L^{2}\left(Q ; \mathbb{R}^{3}\right)} \leqq C, \\
& \left\|\bar{\omega}_{\tau}\right\|_{L^{\infty}\left(Q ; \mathbb{R}^{L}\right)} \leqq C,
\end{aligned}
$$




$$
\begin{aligned}
& \left\|\nabla \bar{\vartheta}_{\tau}\right\|_{L^{r}\left(Q ; \mathbb{R}^{d}\right)} \leqq C_{r} \quad \text { with } r<5 / 4, \\
& \left\|\mathrm{E}\left(\bar{u}_{\tau}\right)\right\|_{L^{\infty}\left(I ; W^{1, \eta}\left(\Omega ; \mathbb{R}_{\mathrm{sym}}^{3 \times 3}\right)\right)} \leqq C \tau^{-1 / \eta}, \\
& \left\|m_{\tau}\right\|_{L^{\infty}\left(I ; W^{1, \eta}\left(\Omega ; \mathbb{R}^{3}\right)\right)} \leqq C \tau^{-1 / \eta}, \\
& \left\|e_{\tau}\right\|_{L^{\eta}\left(Q ; \mathbb{R}^{3}\right)} \leqq C \tau^{-1 / \eta},
\end{aligned}
$$

with some $C$ and $C_{r}$ independent of $\tau$.

Proof. We test the particular equations/inclusion of (77), respectively, by $\frac{u_{\tau}^{k}-u_{\tau}^{k-1}}{\tau}$, $\frac{m_{\tau}^{k}-m_{\tau}^{k-1}}{\tau}, \frac{1}{2}, h_{\tau}^{k}-h_{\mathrm{b}, \tau}^{k}, e_{\tau}^{k}$, and 0 . We use the semiconvexity of $\phi$, and hence the convexity of the functional

$$
\mathfrak{q} \equiv(\mathrm{E}, m) \mapsto \phi(\mathfrak{q})+\frac{\mathbb{D E}: \mathrm{E}+\alpha|m|^{2}}{2 \sqrt{\tau}}+\frac{\tau}{\eta}|\mathrm{E}|^{\eta}+\frac{\tau}{\eta}|m|^{\eta}
$$

for sufficiently small $\tau$, namely for $\tau \leqq\left(\min _{|\mathrm{E}|=1}\left(\mathbb{D E}: \mathrm{E}+\alpha|m|^{2}\right) /\left(4 C_{\phi}\right)\right)^{2}$ with $C_{\phi}$ from (72b) if $C_{\phi}>0$. Then, estimating jointly (77a) and (77b) we have a discrete chain rule at our disposal:

$$
\begin{aligned}
& \left(\phi_{\mathrm{E}}^{\prime}\left(\mathfrak{q}_{\tau}^{k}\right)+\mathbb{D} \mathrm{E}\left(\frac{u_{\tau}^{k}-u_{\tau}^{k-1}}{\tau}\right)+\tau\left|\mathrm{E}\left(u_{\tau}^{k}\right)\right|^{\eta-2} \mathrm{E}\left(u_{\tau}^{k}\right)\right): \mathrm{E}\left(\frac{u_{\tau}^{k}-u_{\tau}^{k-1}}{\tau}\right) \\
& \left.+\left(\phi_{m}^{\prime}\left(\mathfrak{q}_{\tau}^{k}\right)+\alpha \frac{m_{\tau}^{k}-m_{\tau}^{k-1}}{\tau}\right)+\tau\left|m_{\tau}^{k}\right|^{\eta-2} m_{\tau}^{k}\right) \cdot \frac{m_{\tau}^{k}-m_{\tau}^{k-1}}{\tau} \\
& =\left(\phi_{\mathrm{E}}^{\prime}\left(\mathfrak{q}_{\tau}^{k}\right)+\frac{1}{\sqrt{\tau}} \mathbb{D} \mathrm{E}\left(u_{\tau}^{k}\right)+\tau\left|\mathrm{E}\left(u_{\tau}^{k}\right)\right|^{\eta-2} \mathrm{E}\left(u_{\tau}^{k}\right)\right): \mathrm{E}\left(\frac{u_{\tau}^{k}-u_{\tau}^{k-1}}{\tau}\right) \\
& +\left(\phi_{m}^{\prime}\left(\mathfrak{q}_{\tau}^{k}\right)+\frac{1}{\sqrt{\tau}} \alpha m_{\tau}^{k}+\tau\left|m_{\tau}^{k}\right|^{\eta-2} m_{\tau}^{k}\right) \cdot \frac{m_{\tau}^{k}-m_{\tau}^{k-1}}{\tau} \\
& -\frac{1}{\sqrt{\tau}} \mathbb{D} \mathrm{E}\left(u_{\tau}^{k-1}\right): \mathrm{E}\left(\frac{u_{\tau}^{k}-u_{\tau}^{k-1}}{\tau}\right)+(1-\sqrt{\tau}) \mathbb{D} \mathrm{E}\left(\frac{u_{\tau}^{k}-u_{\tau}^{k-1}}{\tau}\right): \mathrm{E}\left(\frac{u_{\tau}^{k}-u_{\tau}^{k-1}}{\tau}\right) \\
& -\frac{1}{\sqrt{\tau}} \alpha m_{\tau}^{k-1} \cdot \frac{m_{\tau}^{k}-m_{\tau}^{k-1}}{\tau}+(1-\sqrt{\tau}) \alpha\left|\frac{m_{\tau}^{k}-m_{\tau}^{k-1}}{\tau}\right|^{2} \\
& \geqq \frac{1}{\tau}\left(\phi\left(\mathfrak{q}_{\tau}^{k}\right)+\frac{\mathbb{D} \mathrm{E}\left(u_{\tau}^{k}\right): \mathrm{E}\left(u_{\tau}^{k}\right)+\alpha\left|m_{\tau}^{k}\right|^{2}}{2 \sqrt{\tau}}+\frac{\tau}{\eta}\left|\mathrm{E}\left(u_{\tau}^{k}\right)\right|^{\eta}+\frac{\tau}{\eta}\left|m_{\tau}^{k}\right|^{\eta}\right. \\
& \left.-\phi\left(\mathfrak{q}_{\tau}^{k-1}\right)-\frac{\mathbb{D} \mathrm{E}\left(u_{\tau}^{k-1}\right): \mathrm{E}\left(u_{\tau}^{k-1}\right)+\alpha\left|m_{\tau}^{k-1}\right|^{2}}{2 \sqrt{\tau}}-\frac{\tau}{\eta}\left|\mathrm{E}\left(u_{\tau}^{k-1}\right)\right|^{\eta}-\frac{\tau}{\eta}\left|m_{\tau}^{k-1}\right|^{\eta}\right) \\
& -\frac{1}{\sqrt{\tau}} \mathbb{D} \mathrm{E}\left(u_{\tau}^{k-1}\right): \mathrm{E}\left(\frac{u_{\tau}^{k}-u_{\tau}^{k-1}}{\tau}\right)+(1-\sqrt{\tau}) \mathbb{D} \mathrm{E}\left(\frac{u_{\tau}^{k}-u_{\tau}^{k-1}}{\tau}\right): \mathrm{E}\left(\frac{u_{\tau}^{k}-u_{\tau}^{k-1}}{\tau}\right) \\
& -\frac{1}{\sqrt{\tau}} \alpha m_{\tau}^{k-1} \cdot \frac{m_{\tau}^{k}-m_{\tau}^{k-1}}{\tau}+(1-\sqrt{\tau}) \alpha\left|\frac{m_{\tau}^{k}-m_{\tau}^{k-1}}{\tau}\right|^{2} \\
& =\frac{\phi\left(\mathfrak{q}_{\tau}^{k}\right)-\phi\left(\mathfrak{q}_{\tau}^{k-1}\right)}{\tau}+\frac{\left|\mathrm{E}\left(u_{\tau}^{k}\right)\right|^{\eta}-\left|\mathrm{E}\left(u_{\tau}^{k-1}\right)\right|^{\eta}}{\eta}+\frac{\left|m_{\tau}^{k}\right|^{\eta}-\left|m_{\tau}^{k-1}\right|^{\eta}}{\eta} \\
& +\left(1-\frac{\sqrt{\tau}}{2}\right)\left(\mathbb{D} \mathrm{E}\left(\frac{u_{\tau}^{k}-u_{\tau}^{k-1}}{\tau}\right): \mathrm{E}\left(\frac{u_{\tau}^{k}-u_{\tau}^{k-1}}{\tau}\right)+\alpha\left|\frac{m_{\tau}^{k}-m_{\tau}^{k-1}}{\tau}\right|^{2}\right),
\end{aligned}
$$


provided $\tau$ is sufficiently small as already specified. Similarly, and even more simply, we use the convexity of the kinetic energy $\frac{\varrho}{2}|\cdot|^{2}$ and of $\nabla \mathrm{E} \mapsto \frac{1}{2} \mathbb{C}_{\mathrm{hpr}} \nabla \mathrm{E}: \nabla \mathrm{E}+$ $\frac{\tau}{\eta}|\nabla \mathrm{E}|^{\eta}$, and also of $\nabla m \mapsto \frac{\lambda}{2}|\nabla m|^{2}$. The gyroscopic term again vanishes, that is, $\frac{m_{\tau}^{k}}{\gamma\left(\left|m_{\tau}^{k}\right|\right)} \times \frac{m_{\tau}^{k}-m_{\tau}^{k-1}}{\tau} \cdot m_{\tau}^{k}=0$, now due to the orthogonality of $m_{\tau}^{k} \times\left(m_{\tau}^{k}-m_{\tau}^{k-1}\right)$ and $m_{\tau}^{k}-m_{\tau}^{k-1}$. Therefore, this joint test of the magneto-mechanical part (77a) and (77b) gives

$$
\begin{aligned}
& \int_{\Omega} \frac{\varrho}{2 \tau}\left(\left|\frac{u_{\tau}^{k}-u_{\tau}^{k-1}}{\tau}\right|^{2}-\left|\frac{u_{\tau}^{k-1}-u_{\tau}^{k-2}}{\tau}\right|^{2}\right) \\
&+\frac{\phi\left(\mathfrak{q}_{\tau}^{k}\right)-\phi\left(\mathfrak{q}_{\tau}^{k-1}\right)}{\tau}+\frac{\lambda\left|\nabla m_{\tau}^{k}\right|^{2}-\lambda\left|\nabla m_{\tau}^{k-1}\right|^{2}}{2 \tau} \\
&+\left(1-\frac{\sqrt{\tau}}{2}\right)\left(\mathbb{D} \mathrm{E}\left(\frac{u_{\tau}^{k}-u_{\tau}^{k-1}}{\tau}\right): \mathrm{E}\left(\frac{u_{\tau}^{k}-u_{\tau}^{k-1}}{\tau}\right)+\alpha\left|\frac{m_{\tau}^{k}-m_{\tau}^{k-1}}{\tau}\right|^{2}\right) \\
&+\frac{\mathbb{C}_{\mathrm{hpr}} \nabla \mathrm{E}\left(u_{\tau}^{k}\right) \vdots \nabla \mathrm{E}\left(u_{\tau}^{k}\right)-\mathbb{C}_{\mathrm{hpr}} \nabla \mathrm{E}\left(u_{\tau}^{k-1}\right) \vdots \nabla \mathrm{E}\left(u_{\tau}^{k-1}\right)}{2 \tau} \\
&+\mathbb{D}_{\mathrm{hpr}} \nabla \mathrm{E}\left(\frac{\left.u_{\tau}^{k}-u_{\tau}^{k-1}\right)}{\tau}\right): \nabla \mathrm{E}\left(\frac{u_{\tau}^{k}-u_{\tau}^{k-1}}{\tau}\right) \\
&+\mathscr{A}_{1}\left(\mathfrak{q}_{\tau}^{k}, \vartheta_{\tau}^{k}\right): \mathrm{E}\left(\frac{u_{\tau}^{k}-u_{\tau}^{k-1}}{\tau}\right)+\mathscr{A}_{2}\left(\mathfrak{q}_{\tau}^{k}, \vartheta_{\tau}^{k}\right) \cdot \frac{m_{\tau}^{k}-m_{\tau}^{k-1}}{\tau} \\
&+\frac{\left|\mathrm{E}\left(u_{\tau}^{k}\right)\right|^{\eta}-\left|\mathrm{E}\left(u_{\tau}^{k-1}\right)\right|^{\eta}}{\eta}+\frac{\left|\nabla \mathrm{E}\left(u_{\tau}^{k}\right)\right|^{\eta}-\left|\nabla \mathrm{E}\left(u_{\tau}^{k-1}\right)\right|^{\eta}}{\eta}+\frac{\left|m_{\tau}^{k}\right|^{\eta}-\left|m_{\tau}^{k-1}\right|^{\eta}}{\eta} \\
&+\chi_{\mathrm{E}}^{\prime}\left(\mathfrak{q}_{\tau}^{k-1}\right)^{\top} \omega_{\tau}^{k}: \mathrm{E}\left(\frac{u_{\tau}^{k}-u_{\tau}^{k-1}}{\tau}\right)+\chi_{m}^{\prime}\left(\mathfrak{q}_{\tau}^{k-1}\right)^{\top} \omega_{\tau}^{k} \cdot \frac{m_{\tau}^{k}-m_{\tau}^{k-1}}{\tau} \\
&= \int_{\Omega}\left(f_{\tau}^{k}-\mu_{0}\left(\nabla h_{\tau}^{k}\right)^{\top} m_{\tau}^{k}\right) \cdot \frac{u_{\tau}^{k}-u_{\tau}^{k-1}}{\tau} \\
&+\mu_{0} h_{\tau}^{k} \cdot \frac{m_{\tau}^{k}-m_{\tau}^{k-1}}{\tau} \mathrm{d} x+\int_{\Gamma} g_{\tau}^{k} \cdot \frac{u_{\tau}^{k}-u_{\tau}^{k-1}}{\tau} \mathrm{d} S . \\
& \tau
\end{aligned}
$$

In the Maxwell system, we test (77d) by $h_{\tau}^{k}-h_{\mathrm{b}, \tau}^{k}$ and use the convexity of $h \mapsto$ $\frac{1}{2} \mu_{0}|h|^{2}$, which yields

$$
\begin{aligned}
& \int_{\Omega} \mu_{0} \frac{\left|h_{\tau}^{k}\right|^{2}-\left|h_{\tau}^{k-1}\right|^{2}}{2 \tau}+\mu_{0}\left(\frac{m_{\tau}^{k}-m_{\tau}^{k-1}}{\tau}-\nabla m_{\tau}^{k} \frac{u_{\tau}^{k}-u_{\tau}^{k-1}}{\tau}-\operatorname{div} \frac{u_{\tau}^{k}-u_{\tau}^{k-1}}{\tau} m_{\tau}^{k}\right) \cdot h_{\tau}^{k} \\
& \quad+\operatorname{curl} e_{\tau}^{k} \cdot\left(h_{\tau}^{k}-h_{\tau}^{k-1}\right) \mathrm{d} x \\
& \leqq \int_{\Omega} \mu_{0}\left(\frac{h_{\tau}^{k}-h_{\tau}^{k-1}}{\tau}+\frac{m_{\tau}^{k}-m_{\tau}^{k-1}}{\tau}-\nabla m_{\tau}^{k} \frac{u_{\tau}^{k}-u_{\tau}^{k-1}}{\tau}-\operatorname{div} \frac{u_{\tau}^{k}-u_{\tau}^{k-1}}{\tau} m_{\tau}^{k}\right) \cdot h_{\mathrm{b}, \tau}^{k} \mathrm{~d} x .
\end{aligned}
$$

Moreover, we test (77e) by $e_{\tau}^{k}$, which gives

$$
\int_{\Omega} \mathscr{S}\left(\mathfrak{q}_{\tau}^{k}, \vartheta_{\tau}^{k}\right) e_{\tau}^{k}: e_{\tau}^{k}+\tau\left|e_{\tau}^{k}\right|^{\eta}-\operatorname{curl}\left(h_{\tau}^{k}-h_{\mathrm{b}, \tau}^{k}\right) \cdot e_{\tau}^{k} \mathrm{~d} x=0 .
$$


The mentioned test of (77f) in the weak formulation, taking 0-homogeneity of $\partial \zeta$ into account, that is,

$$
\zeta(v)-\omega_{\tau}^{k} \cdot\left(v-\chi^{\prime}\left(\mathfrak{q}_{\tau}^{k-1}\right) \frac{\mathfrak{q}_{\tau}^{k}-\mathfrak{q}_{\tau}^{k-1}}{\tau}\right) \geqq \zeta\left(\chi^{\prime}\left(\mathfrak{q}_{\tau}^{k-1}\right) \frac{\mathfrak{q}_{\tau}^{k}-\mathfrak{q}_{\tau}^{k-1}}{\tau}\right),
$$

for $v=0$ gives

$$
\begin{aligned}
\zeta\left(\chi^{\prime}\left(\mathfrak{q}_{\tau}^{k-1}\right) \frac{\mathfrak{q}_{\tau}^{k}-\mathfrak{q}_{\tau}^{k-1}}{\tau}\right) \leqq & \chi_{\mathrm{E}}^{\prime}\left(\mathfrak{q}_{\tau}^{k-1}\right)^{\top} \omega_{\tau}^{k}: \mathrm{E}\left(\frac{u_{\tau}^{k}-u_{\tau}^{k-1}}{\tau}\right) \\
& +\chi_{m}^{\prime}\left(\mathfrak{q}_{\tau}^{k-1}\right)^{\top} \omega_{\tau}^{k} \cdot \frac{m_{\tau}^{k}-m_{\tau}^{k-1}}{\tau} .
\end{aligned}
$$

Then, we can sum (104), (105), (106), and (108), and again use the cancellation of the magnetostrictive terms (84) and the curl-cancellation (7) now with the boundary term vanishing, since $n \times\left(h_{\tau}^{k}-h_{\mathrm{b}, \tau}^{k}\right)=0$ on $\Gamma$. The terms with $\omega_{\tau}^{k}$, as well as the terms $\pm \frac{m_{\tau}^{l}-m_{\tau}^{l-1}}{\tau} \cdot h_{\tau}^{l}$, mutually cancel. Thus, summing up over the time levels $l=1, \ldots, k$ and using (45) and (73), we arrive at the following estimate for the electro-magneto-mechanical energy

$$
\begin{aligned}
& \sum_{l=1}^{k} \int_{\Omega}\left(1-\frac{\sqrt{\tau}}{2}\right)\left(\mathbb{D E}\left(\frac{u_{\tau}^{l}-u_{\tau}^{l-1}}{\tau}\right): \mathrm{E}\left(\frac{u_{\tau}^{l}-u_{\tau}^{l-1}}{\tau}\right)+\alpha\left|\frac{m_{\tau}^{l}-m_{\tau}^{l-1}}{\tau}\right|^{2}\right) \\
& +\mathbb{D}_{\mathrm{hpr}} \nabla \mathrm{E}\left(\frac{u_{\tau}^{l}-u_{\tau}^{l-1}}{\tau}\right) \vdots \nabla \mathrm{E}\left(\frac{u_{\tau}^{l}-u_{\tau}^{l-1}}{\tau}\right)+\mathscr{S}\left(\mathfrak{q}_{\tau}^{l}, \vartheta_{\tau}^{l}\right) e_{\tau}^{l}: e_{\tau}^{l}+\tau\left|e_{\tau}^{l}\right|^{\eta} \\
& +\zeta\left(\chi^{\prime}\left(\mathfrak{q}_{\tau}^{l-1}\right) \frac{\mathfrak{q}_{\tau}^{l}-\mathfrak{q}_{\tau}^{l-1}}{\tau}\right)+\mathscr{A}\left(\mathfrak{q}_{\tau}^{l}, \vartheta_{\tau}^{l}\right): \frac{\mathfrak{q}_{\tau}^{l}-\mathfrak{q}_{\tau}^{l-1}}{\tau} \mathrm{d} x \\
& +\Phi\left(u_{\tau}^{k}, \frac{u_{\tau}^{k}-u_{\tau}^{k-1}}{\tau}, m_{\tau}^{k}, h_{\tau}^{k}\right)+\int_{\Omega} \frac{\tau}{\eta}\left|\mathrm{E}\left(u_{\tau}^{k}\right)\right|^{\eta}+\frac{\tau}{\eta}\left|\nabla \mathrm{E}\left(u_{\tau}^{k}\right)\right|^{\eta}+\frac{\tau}{\eta}\left|m_{\tau}^{k}\right|^{\eta} \mathrm{d} x \\
& \leqq \tau \sum_{l=1}^{k}\left(\int_{\Omega} f_{\tau}^{l} \cdot \frac{u_{\tau}^{l}-u_{\tau}^{l-1}}{\tau}+\operatorname{curl} h_{\mathrm{b}, \tau}^{l} \cdot e_{\tau}^{l}+\mu_{0}\left(\frac{h_{\tau}^{l}-h_{\tau}^{l-1}}{\tau}+\frac{m_{\tau}^{l}-m_{\tau}^{l-1}}{\tau}\right.\right. \\
& \left.\left.-\nabla m_{\tau}^{l} \frac{u_{\tau}^{l}-u_{\tau}^{l-1}}{\tau}-\operatorname{div} \frac{u_{\tau}^{l}-u_{\tau}^{l-1}}{\tau} m_{\tau}^{l}\right) \cdot h_{\mathrm{b}, \tau}^{l} \mathrm{~d} x+\int_{\Gamma} g_{\tau}^{l} \cdot \frac{u_{\tau}^{l}-u_{\tau}^{l-1}}{\tau} \mathrm{d} S\right) \\
& +\Phi\left(u_{0 \tau}, v_{0}, m_{0 \tau}, h_{0}\right)+\int_{\Omega} \frac{\tau}{\eta}\left|\mathrm{E}\left(u_{0 \tau}\right)\right|^{\eta}+\frac{\tau}{\eta}\left|\nabla \mathrm{E}\left(u_{0 \tau}\right)\right|^{\eta}+\frac{\tau}{\eta}\left|m_{0 \tau}\right|^{\eta} \mathrm{d} x .
\end{aligned}
$$

This is a discrete analog of (49)-(51) with the additional regularizing " $\eta$-terms".

We still add the heat part (77c) tested by $\frac{1}{2}$ to see $\left\|\vartheta_{\tau}^{k}\right\|_{L^{1}(\Omega)}$, but simultaneously to keep (one half of) the dissipation terms in the left-hand side. We thus arrive at the the following estimate: 


$$
\begin{aligned}
\Phi( & \left.u_{\tau}^{k}, \frac{u_{\tau}^{k}-u_{\tau}^{k-1}}{\tau}, m_{\tau}^{k}, h_{\tau}^{k}\right)+\frac{1}{2}\left\|\vartheta_{\tau}^{k}\right\|_{L^{1}(\Omega)} \\
& +\sum_{l=1}^{k} \int_{\Omega}\left(\frac{1}{2}-\frac{\sqrt{\tau}}{4}\right)\left(\mathbb{D E}\left(\frac{u_{\tau}^{l}-u_{\tau}^{l-1}}{\tau}\right): \mathrm{E}\left(\frac{u_{\tau}^{l}-u_{\tau}^{l-1}}{\tau}\right)+\alpha\left|\frac{m_{\tau}^{l}-m_{\tau}^{l-1}}{\tau}\right|^{2}\right) \\
& +\frac{1}{2} \mathbb{D}_{\mathrm{hpr}} \nabla \mathrm{E}\left(\frac{u_{\tau}^{l}-u_{\tau}^{l-1}}{\tau}\right) \vdots \nabla \mathrm{E}\left(\frac{u_{\tau}^{l}-u_{\tau}^{l-1}}{\tau}\right)+\frac{1}{2} \mathscr{S}\left(\mathfrak{q}_{\tau}^{l}, \vartheta_{\tau}^{l}\right) e_{\tau}^{l}: e_{\tau}^{l}+\tau\left|e_{\tau}^{l}\right|^{\eta} \\
& +\frac{1}{2} \zeta\left(\chi^{\prime}\left(\mathfrak{q}_{\tau}^{l-1}\right) \frac{\mathfrak{q}_{\tau}^{l}-\mathfrak{q}_{\tau}^{l-1}}{\tau}\right)+\frac{1}{2} \mathscr{A}\left(\mathfrak{q}_{\tau}^{l}, \vartheta_{\tau}^{l}\right): \frac{\mathfrak{q}_{\tau}^{l}-\mathfrak{q}_{\tau}^{l-1}}{\tau} \mathrm{d} x \\
& +\int_{\Omega} \frac{\tau}{\eta}\left|\mathrm{E}\left(u_{\tau}^{k}\right)\right|^{\eta}+\frac{\tau}{\eta}\left|\nabla \mathrm{E}\left(u_{\tau}^{k}\right)\right|^{\eta}+\frac{\tau}{\eta}\left|m_{\tau}^{k}\right|^{\eta} \mathrm{d} x+\sum_{l=1}^{k} \int_{\Omega} \tau\left|e_{\tau}^{l}\right|^{\eta} \mathrm{d} x \\
\leqq & \tau \sum_{l=1}^{k}\left(\int_{\Omega} f_{\tau}^{l} \cdot \frac{u_{\tau}^{l}-u_{\tau}^{l-1}}{\tau}+\operatorname{curl} h_{\mathrm{b}, \tau}^{l} \cdot e_{\tau}^{l}+\mu_{0}\left(\frac{h_{\tau}^{l}-h_{\tau}^{l-1}}{\tau}+\frac{m_{\tau}^{l}-m_{\tau}^{l-1}}{\tau}\right.\right. \\
& \left.\left.-\nabla m_{\tau}^{l} \frac{u_{\tau}^{l}-u_{\tau}^{l-1}}{\tau}-\operatorname{div} \frac{u_{\tau}^{l}-u_{\tau}^{l-1}}{\tau} m_{\tau}^{l}\right) \cdot h_{\mathrm{b}, \tau}^{l} \mathrm{~d} x+\int_{\Gamma} g_{\tau}^{l} \cdot \frac{u_{\tau}^{l}-u_{\tau}^{l-1}}{\tau} q_{\mathrm{b}, \tau}^{l} \mathrm{~d} S\right) \\
& +\Phi\left(u_{0 \tau}, v_{0}, m_{0 \tau}, h_{0}\right)+\left\|\hat{c}_{\mathrm{v}}\left(\mathrm{E}\left(u_{0 \tau}\right), \theta_{0}\right)\right\|_{L^{1}(\Omega)} \\
& +\int_{\Omega} \frac{\tau}{\eta}\left|\mathrm{E}\left(u_{0 \tau}\right)\right|^{\eta}+\frac{\tau}{\eta}\left|\nabla \mathrm{E}\left(u_{0 \tau}\right)\right|^{\eta}+\frac{\tau}{\eta}\left|m_{0 \tau}\right|^{\eta} \mathrm{d} x .
\end{aligned}
$$

To get the a priori estimates by the discrete Gronwall inequality, we must still treat the $h_{\mathrm{b}}$-terms carefully. First, we use the by-part summation formula

$$
\tau \sum_{l=1}^{k} \frac{h_{\tau}^{l}-h_{\tau}^{l-1}}{\tau} \cdot h_{\mathrm{b}, \tau}^{l}=h_{\tau}^{k} \cdot h_{\mathrm{b}, \tau}^{k}-h_{0} \cdot h_{\mathrm{b}, \tau}^{1}-\tau \sum_{l=1}^{k} h_{\tau}^{l-1} \cdot \frac{h_{\mathrm{b}, \tau}^{l}-h_{\mathrm{b}, \tau}^{l-1}}{\tau}
$$

and estimate $\left|\int_{\Omega} h_{\tau}^{l-1} \cdot \frac{h_{\mathrm{b}, \tau}^{l}-h_{\mathrm{b}, \tau}^{l-1}}{\tau} \mathrm{d} x\right| \leqq\left(1+\left\|h_{\tau}^{l-1}\right\|_{L^{2}\left(\Omega ; \mathbb{R}^{3}\right)}^{2}\right)\left\|\frac{h_{\mathrm{b}, \tau}^{l}-h_{\mathrm{b}, \tau}^{l-1}}{\tau}\right\|_{L^{2}\left(\Omega ; \mathbb{R}^{3}\right)}$, which needs $h_{\mathrm{b}} \in W^{1,1}\left(I ; L^{2}\left(\Omega ; \mathbb{R}^{3}\right)\right)$. The same treatment applies to the term $\frac{m_{\tau}^{l}-m_{\tau}^{l-1}}{\tau} \cdot h_{\mathrm{b}, \tau}^{l}$. The other two $h_{\mathrm{b}}$-terms can be estimated by using (35) as

$$
\begin{aligned}
& \int_{\Omega}\left(-\nabla m_{\tau}^{l} \frac{u_{\tau}^{l}-u_{\tau}^{l-1}}{\tau}-\operatorname{div} \frac{u_{\tau}^{l}-u_{\tau}^{l-1}}{\tau} m_{\tau}^{l}\right) \cdot h_{\mathrm{b}, \tau}^{l} \mathrm{~d} x \\
& =\int_{\Omega}\left(\nabla h_{\mathrm{b}, \tau}^{l}\right)^{\top} m_{\tau}^{l} \frac{u_{\tau}^{l}-u_{\tau}^{l-1}}{\tau} \mathrm{d} x-\int_{\Gamma}\left(m_{\tau}^{l} \cdot h_{\mathrm{b}, \tau}^{l}\right) \frac{u_{\tau}^{l}-u_{\tau}^{l-1}}{\tau} \cdot n \mathrm{~d} S \\
& \leqq\left\|\nabla h_{\mathrm{b}, \tau}^{l}\right\|_{L^{\infty}\left(\Omega ; \mathbb{R}^{3}\right)}\left\|m_{\tau}^{l}\right\|_{L^{2}\left(\Omega ; \mathbb{R}^{3}\right)}\left\|\frac{u_{\tau}^{l}-u_{\tau}^{l-1}}{\tau}\right\|_{L^{2}\left(\Omega ; \mathbb{R}^{3}\right)} \\
& +\left\|h_{\mathrm{b}, \tau}^{l}\right\|_{L^{\infty}\left(\Gamma ; \mathbb{R}^{3}\right)}\left\|m_{\tau}^{l}\right\|_{L^{2}\left(\Gamma ; \mathbb{R}^{3}\right)}\left\|\frac{u_{\tau}^{l}-u_{\tau}^{l-1}}{\tau}\right\|_{L^{2}\left(\Gamma ; \mathbb{R}^{3}\right)} \\
& \leqq \frac{1}{4}\left\|\frac{u_{\tau}^{l}-u_{\tau}^{l-1}}{\tau}\right\|_{L^{2}\left(\Omega ; \mathbb{R}^{3}\right)}^{2}+\left\|\nabla h_{\mathrm{b}, \tau}^{l}\right\|_{L^{\infty}\left(\Omega ; \mathbb{R}^{3}\right)}^{2}\left\|m_{\tau}^{l}\right\|_{L^{2}\left(\Gamma ; \mathbb{R}^{3}\right)}^{2} \\
& +\delta\left\|\frac{u_{\tau}^{l}-u_{\tau}^{l-1}}{\tau}\right\|_{L^{2}\left(\Gamma ; \mathbb{R}^{3}\right)}^{2}+\frac{1}{4 \delta}\left\|h_{\mathrm{b}, \tau}^{l}\right\|_{L^{\infty}\left(\Gamma ; \mathbb{R}^{3}\right)}^{2}\left\|m_{\tau}^{l}\right\|_{L^{2}\left(\Gamma ; \mathbb{R}^{3}\right)}^{2},
\end{aligned}
$$




$$
\begin{aligned}
\leqq & \frac{1}{4}\left\|\frac{u_{\tau}^{l}-u_{\tau}^{l-1}}{\tau}\right\|_{L^{2}\left(\Omega ; \mathbb{R}^{3}\right)}^{2}+\frac{1}{\varepsilon}\left\|\nabla h_{\mathrm{b}, \tau}^{l}\right\|_{L^{\infty}\left(\Omega ; \mathbb{R}^{3}\right)}^{2} \int_{\Omega} \phi\left(\mathrm{E}\left(u_{\tau}^{l}\right), m_{\tau}^{l}\right) \mathrm{d} x \\
& +\delta\left\|\frac{u_{\tau}^{l}-u_{\tau}^{l-1}}{\tau}\right\|_{L^{2}\left(\Gamma ; \mathbb{R}^{3}\right)}^{2}+\frac{N^{2}}{4 \delta}\left\|h_{\mathrm{b}, \tau}^{l}\right\|_{L^{\infty}\left(\Gamma ; \mathbb{R}^{3}\right)}^{2}\left\|\nabla m_{\tau}^{l}\right\|_{L^{2}\left(\Omega ; \mathbb{R}^{3 \times 3}\right)}^{2} \\
& +\frac{N^{2}}{4 \delta \varepsilon}\left\|h_{\mathrm{b}, \tau}^{l}\right\|_{L^{\infty}\left(\Gamma ; \mathbb{R}^{3}\right)}^{2} \int_{\Omega} \phi\left(\mathrm{E}\left(u_{\tau}^{l}\right), m_{\tau}^{l}\right) \mathrm{d} x,
\end{aligned}
$$

where $\varepsilon$ comes from (72d) and $N$ is the norm of the trace operator $\left.m \mapsto m\right|_{\Gamma}$ : $W^{1,2}(\Omega) \rightarrow L^{2}(\Gamma)$. All the terms on the right-hand side can be treated by the discrete Gronwall inequality except the third term, which can be absorbed in the left-hand side of (110) if $\delta>0$ is chosen sufficiently small, depending on the positive-definiteness of $\mathbb{D}$.

The $\mathscr{A}$-term in (110) can be estimated as

$$
\begin{aligned}
& \left|\int_{\Omega} \mathscr{A}\left(\mathfrak{q}_{\tau}^{l}, \vartheta_{\tau}^{l}\right): \frac{\mathfrak{q}_{\tau}^{l}-\mathfrak{q}_{\tau}^{l-1}}{\tau} \mathrm{d} x\right| \\
& \quad \leqq\left\|\mathscr{A}\left(\mathfrak{q}_{\tau}^{l}, \vartheta_{\tau}^{l}\right)\right\|_{L^{2}\left(\Omega ; \mathbb{R}^{3 \times 3+3}\right)}\left\|\frac{\mathfrak{q}_{\tau}^{l}-\mathfrak{q}_{\tau}^{l-1}}{\tau}\right\|_{L^{2}\left(\Omega ; \mathbb{R}^{3 \times 3+3}\right)} \\
& \quad \leqq \frac{1}{4 \delta} C_{\mathscr{A}}^{2}\left(1+\int_{\Omega} \phi\left(\mathrm{E}\left(u_{\tau}^{l}\right), m_{\tau}^{l}\right)+\vartheta_{\tau}^{l} \mathrm{~d} x\right)+\delta\left\|\frac{\mathfrak{q}_{\tau}^{l}-\mathfrak{q}_{\tau}^{l-1}}{\tau}\right\|_{L^{2}\left(\Omega ; \mathbb{R}^{3 \times 3+3}\right)}^{2},
\end{aligned}
$$

where $C_{\mathscr{A}}$ is from $(72 \mathrm{~h})$, and then treated again by the discrete Gronwall inequality after absorbing the last term in the left-hand side of (110), if $\delta>0$ is chosen sufficiently small depending on the positive-definiteness of $\mathbb{D}$ and on $\lambda$.

In this way, we obtain all the estimates (102) except (102f) and (102g).

As for (102f), it follows simply from boundedness of $\partial \zeta \subset \mathbb{R}^{L}$.

To obtain $(102 \mathrm{~g})$, we define $\varpi(\vartheta):=\left((1+\vartheta)^{1-\varepsilon}-1\right) /(\varepsilon-1)$ and test $(77 \mathrm{c})$ by $\varpi^{\prime}\left(\vartheta_{\tau}^{k}\right)$. Note that $\varpi(0)=0$ and is convex, and thus $\varpi\left(\vartheta_{\tau}^{k}\right)-\varpi\left(\vartheta_{\tau}^{k-1}\right) \leqq$ $\left(\vartheta_{\tau}^{k}-\vartheta_{\tau}^{k-1}\right) \varpi^{\prime}\left(\vartheta_{\tau}^{k}\right)$. Using this, after summation of (77c) tested by $\varpi^{\prime}\left(\vartheta_{\tau}^{k}\right)$ for $k=1, \ldots, T / \tau$, we obtain

$$
\begin{aligned}
& \kappa_{0} \varepsilon \int_{Q} \frac{\left|\nabla \bar{\vartheta}_{\tau}\right|^{2}}{\left(1+\bar{\vartheta}_{\tau}\right)^{1+\varepsilon}} \mathrm{d} x \mathrm{~d} t \\
& =\kappa_{0} \int_{Q} \varpi^{\prime \prime}\left(\bar{\vartheta}_{\tau}\right)\left|\nabla \bar{\vartheta}_{\tau}\right|^{2} \mathrm{~d} x \mathrm{~d} t \\
& \leqq \int_{Q} \varpi^{\prime \prime}\left(\bar{\vartheta}_{\tau}\right) \mathscr{K}_{0}\left(\overline{\mathfrak{q}}_{\tau}, \bar{\vartheta}_{\tau}\right) \nabla \bar{\vartheta}_{\tau} \cdot \nabla \bar{\vartheta}_{\tau} \mathrm{d} x \mathrm{~d} t \\
& =\int_{Q} \mathscr{K}_{0}\left(\overline{\mathfrak{q}}_{\tau}, \bar{\vartheta}_{\tau}\right) \nabla \bar{\vartheta}_{\tau} \cdot \nabla \varpi^{\prime}\left(\bar{\vartheta}_{\tau}\right) \mathrm{d} x \mathrm{~d} t \\
& \leqq \int_{Q} \mathscr{K}_{0}\left(\overline{\mathfrak{q}}_{\tau}, \bar{\vartheta}_{\tau}\right) \nabla \bar{\vartheta}_{\tau} \cdot \nabla \varpi^{\prime}\left(\bar{\vartheta}_{\tau}\right) \mathrm{d} x \mathrm{~d} t+\int_{\Omega} \varpi\left(\bar{\vartheta}_{\tau}(T, \cdot)\right) \mathrm{d} x \\
& \leqq \int_{\Omega} \varpi\left(\vartheta_{0}\right) \mathrm{d} x+\int_{\Sigma} \bar{q}_{\mathrm{b}, \tau} \varpi^{\prime}\left(\bar{\vartheta}_{\tau}\right) \mathrm{d} S \mathrm{~d} t \\
& +\int_{Q} \bar{r}_{\tau} \varpi^{\prime}\left(\bar{\vartheta}_{\tau}\right)-\mathscr{K}\left(\overline{\mathfrak{q}}_{\tau}, \bar{\vartheta}_{\tau}\right) \nabla \overline{\mathfrak{q}}_{\tau} \cdot \nabla \varpi^{\prime}\left(\bar{\vartheta}_{\tau}\right) \mathrm{d} x \mathrm{~d} t
\end{aligned}
$$




$$
\begin{aligned}
\leqq & \left\|\hat{c}\left(\mathrm{E}\left(u_{0}\right), m_{0}, \theta_{0}\right)\right\|_{L^{1}(\Omega)}+\left\|\bar{q}_{\mathrm{b}, \tau}\right\|_{L^{1}(\Sigma)}+\left\|\bar{r}_{\tau}\right\|_{L^{1}(Q)} \\
& +\frac{\varepsilon T C^{2}}{4 \delta}+\varepsilon \delta C_{\mathscr{K}} \int_{Q} \frac{\left|\nabla \bar{\vartheta}_{\tau}\right|^{2}}{\left(1+\bar{\vartheta}_{\tau}\right)^{1+\varepsilon}} \mathrm{d} x \mathrm{~d} t
\end{aligned}
$$

where $\kappa_{0}$ is from $(72 \mathrm{e})$, and where we abbreviated the heat sources

$$
\begin{aligned}
\bar{r}_{\tau}:= & \zeta\left(\dot{m}_{\tau}\right)+\left(1-\frac{\sqrt{\tau}}{2}\right) \operatorname{DE}\left(\dot{u}_{\tau}\right): \mathrm{E}\left(\dot{u}_{\tau}\right)+\mathbb{D}_{\mathrm{hpr}} \nabla \mathrm{E}\left(\dot{u}_{\tau}\right): \nabla \mathrm{E}\left(\dot{u}_{\tau}\right) \\
& +\left(1-\frac{\sqrt{\tau}}{2}\right) \alpha\left|\dot{m}_{\tau}\right|^{2}+\mathscr{S}\left(\overline{\mathfrak{q}}_{\tau}, \bar{\vartheta}_{\tau}\right) \bar{e}_{\tau} \cdot \bar{e}_{\tau}+\mathscr{A}\left(\overline{\mathfrak{q}}_{\tau}, \bar{\vartheta}_{\tau}\right): \dot{\mathfrak{q}}_{\tau} .
\end{aligned}
$$

In the last line of (114), $C$ is from the already proved estimate (102a) and we have used that $\varpi\left(\vartheta_{0}\right) \leqq \vartheta_{0}=: \hat{c}\left(\mathrm{E}\left(u_{0}\right), m_{0}, \theta_{0}\right)$, and that always $0 \leqq \varpi^{\prime} \leqq 1$, as well as

$$
\begin{aligned}
& \int_{Q} \mathscr{K}\left(\overline{\mathfrak{q}}_{\tau}, \bar{\vartheta}_{\tau}\right) \nabla \overline{\mathfrak{q}}_{\tau} \cdot \nabla \bar{\varpi}^{\prime}\left(\bar{\vartheta}_{\tau}\right) \mathrm{d} x \mathrm{~d} t \\
& \quad=\varepsilon \int_{Q} \nabla \overline{\mathfrak{q}}_{\tau}: \frac{\mathscr{K}\left(\overline{\mathfrak{q}}_{\tau}, \bar{\vartheta}_{\tau}\right) \otimes \nabla \bar{\vartheta}_{\tau}}{\left(1+\bar{\vartheta}_{\tau}\right)^{1+\varepsilon}} \mathrm{d} x \mathrm{~d} t \\
& \quad \leqq \varepsilon \int_{Q} \frac{1}{4 \delta}\left|\nabla \overline{\mathfrak{q}}_{\tau}\right|^{2}+\delta \frac{\left|\mathscr{K}\left(\overline{\mathfrak{q}}_{\tau}, \bar{\vartheta}_{\tau}\right)\right|^{2}}{1+\bar{\vartheta}_{\tau}} \frac{\left|\nabla \bar{\vartheta}_{\tau}\right|^{2}}{\left(1+\bar{\vartheta}_{\tau}\right)^{1+\varepsilon}} \mathrm{d} x \mathrm{~d} t \\
& \quad \leqq \varepsilon \int_{Q} \frac{1}{4 \delta}\left|\nabla \overline{\mathfrak{q}}_{\tau}\right|^{2}+\delta C \mathscr{K} \frac{\left|\nabla \bar{\vartheta}_{\tau}\right|^{2}}{\left(1+\bar{\vartheta}_{\tau}\right)^{1+\varepsilon}} \mathrm{d} x \mathrm{~d} t,
\end{aligned}
$$

with $C_{\mathscr{K}}$ from (72g). From the already obtained estimates, we can see that the dissipative-heat terms (included Joule heat) on the right-hand side of the heat equation (101c) are bounded in $L^{1}(Q)$. As $\int_{\Omega} \phi\left(\mathrm{E}\left(u_{\tau}^{l}\right), m_{\tau}^{l}\right)+\vartheta_{\tau}^{l} \mathrm{~d} x$ is shown bounded uniformly in time, from $(72 \mathrm{~h})$ we have $\mathscr{A}\left(\overline{\mathfrak{q}}_{\tau}, \bar{\vartheta}_{\tau}\right)$ bounded in $L^{\infty}\left(I ; L^{2}\left(\Omega ; \mathbb{R}^{3 \times 3+3}\right)\right)$ so that the adiabatic heat $\mathscr{A}\left(\overline{\mathfrak{q}}_{\tau}, \bar{\vartheta}_{\tau}\right): \dot{\mathfrak{q}}_{\tau}$ is bounded, in fact, not only in $L^{1}(Q)$ but also in $L^{2}\left(I ; L^{3 / 2}(\Omega)\right)$. Altogether, $\bar{r}_{\tau}$ from (115) is bounded in $L^{1}(Q)$.

Next, we choose $\delta<\kappa_{0} / C_{\mathscr{K}}$ to absorb the last term in (114) in its first term. Then the usual Gagliardo-Nirenberg interpolation with the already obtained estimate (102c) yields (102g); we refer to [7,8] or to [37, 2nd edn, Sect. 9.4] for details.

Remark 7. (Discrete energy balance.) Adding the heat part $(77 \mathrm{c})$ tested by 1 to (109), we could see the cancellation of the dissipative-heat and the adiabatic terms and would have arrived at the the following estimate for the total energy:

$$
\begin{aligned}
& \Phi\left(u_{\tau}^{k}, \frac{u_{\tau}^{k}-u_{\tau}^{k-1}}{\tau}, m_{\tau}^{k}, h_{\tau}^{k}\right)+\left\|\vartheta_{\tau}^{k}\right\|_{L^{1}(\Omega)} \\
& \quad+\int_{\Omega} \frac{\tau}{\eta}\left|\mathrm{E}\left(u_{\tau}^{k}\right)\right|^{\eta}+\frac{\tau}{\eta}\left|\nabla \mathrm{E}\left(u_{\tau}^{k}\right)\right|^{\eta}+\frac{\tau}{\eta}\left|m_{\tau}^{k}\right|^{\eta} \mathrm{d} x+\sum_{l=1}^{k} \int_{\Omega} \tau\left|e_{\tau}^{l}\right|^{\eta} \mathrm{d} x
\end{aligned}
$$




$$
\begin{aligned}
\leqq & \tau \sum_{l=1}^{k}\left(\int_{\Omega} f_{\tau}^{l} \cdot \frac{u_{\tau}^{l}-u_{\tau}^{l-1}}{\tau}+\operatorname{curl} h_{\mathrm{b}, \tau}^{l} \cdot e_{\tau}^{l}+\mu_{0}\left(\frac{h_{\tau}^{l}-h_{\tau}^{l-1}}{\tau}+\frac{m_{\tau}^{l}-m_{\tau}^{l-1}}{\tau}\right.\right. \\
& \left.\left.-\nabla m_{\tau}^{l} \frac{u_{\tau}^{l}-u_{\tau}^{l-1}}{\tau}-\operatorname{div} \frac{u_{\tau}^{l}-u_{\tau}^{l-1}}{\tau} m_{\tau}^{l}\right) \cdot h_{\mathrm{b}, \tau}^{l} \mathrm{~d} x+\int_{\Gamma} g_{\tau}^{l} \cdot \frac{u_{\tau}^{l}-u_{\tau}^{l-1}}{\tau}+q_{\mathrm{b}, \tau}^{l} \mathrm{~d} S\right) \\
& +\Phi\left(u_{0 \tau}, v_{0}, m_{0 \tau}, h_{0}\right)+\left\|\hat{c}_{\mathrm{v}}\left(\mathrm{E}\left(u_{0 \tau}\right), \theta_{0}\right)\right\|_{L^{1}(\Omega)} \\
& +\int_{\Omega} \frac{\tau}{\eta}\left|\mathrm{E}\left(u_{0 \tau}\right)\right|^{\eta}+\frac{\tau}{\eta}\left|\nabla \mathrm{E}\left(u_{0 \tau}\right)\right|^{\eta}+\frac{\tau}{\eta}\left|m_{0 \tau}\right|^{\eta} \mathrm{d} x .
\end{aligned}
$$

This imitates the energy balance (46), here with the regularizing terms added and again combined with (51). Note that here, to have the mentioned cancellation of the dissipative terms, we benefit from having carefully designed (77c) with the suitable coefficient $1-\sqrt{\tau} / 2$ in front of some dissipative-heat terms.

Remark 8. (Estimates in electrically isolated specimens.) The estimate (117) is usually a point of departure to obtaining estimates $\Phi\left(\bar{u}_{\tau}, \dot{u}_{\tau}, \bar{m}_{\tau}, \bar{h}_{\tau}\right) \in L^{\infty}(I)$ and $\bar{\vartheta}_{\tau} \in L^{\infty}\left(I ; L^{1}(\Omega)\right)$, while the rest of (102) is obtained only afterwards by executing (110) with the already obtained estimates; this allows for sophisticated repeated Gagliardo-Nirenberg interpolation and weakening of the growth hypotheses on $\mathscr{A}$. In this way, the adiabatic heat $\mathscr{A}(\mathfrak{q}, \vartheta): \dot{\mathfrak{q}}$ would be optimally controlled only in $L^{1}(Q)$, instead of its sub-optimal estimate $L^{2}\left(I ; L^{3 / 2}(\Omega)\right)$ obtained by the used technique above. This would, indeed, be possible here if $h_{\mathrm{b}}$ were zero (hence the body would be electrically isolated). Yet, in the general case, (112) combined with (117) does not yield an estimate because $\frac{u_{\tau}^{l}-u_{\tau}^{l-1}}{\tau}$ is not controlled if there is no dissipation in the left-hand side of (117).

Lemma 3. (Further estimates.) Under the assumptions of Lemmas 1 and 2, for some constant $C$ independent of $\tau$, it also holds that

$$
\begin{aligned}
& \left\|\varrho \ddot{u}_{\tau}^{\mathrm{i}}\right\|_{L^{2}\left(I ; W^{2,2}\left(\Omega ; \mathbb{R}^{3}\right)^{*}\right)+L^{\eta^{\prime}}\left(I ; W^{2, \eta}\left(\Omega ; \mathbb{R}^{3}\right)^{*}\right)} \leqq C, \\
& \| \varrho \ddot{u}_{\tau}^{\mathrm{i}}-\tau \operatorname{div}\left(\left|\mathrm{E}\left(\bar{u}_{\tau}\right)\right|^{\eta-2} \mathrm{E}\left(\bar{u}_{\tau}\right)\right) \\
& \quad+\tau \operatorname{div}^{2}\left(\left|\nabla \mathrm{E}\left(\bar{u}_{\tau}\right)\right|^{\eta-2} \nabla \mathrm{E}\left(\bar{u}_{\tau}\right)\right) \|_{L^{2}\left(I ; W^{2,2}\left(\Omega ; \mathbb{R}^{3}\right)^{*}\right)} \leqq C, \\
& \left\|\dot{\vartheta}_{\tau}\right\|_{L^{1}\left(I ; W^{3,2}(\Omega)^{*}\right)} \leqq C, \\
& \left\|\operatorname{curl} \bar{h}_{\tau}+\tau\left|\bar{e}_{\tau}\right|^{\eta-2} \bar{e}_{\tau}\right\|_{\left.L^{2}\left(Q ; \mathbb{R}^{3}\right)\right)} \leqq C, \\
& \left\|\dot{h}_{\tau}\right\|_{L^{2}\left(I ; L_{\text {curl }, 0}^{2}\left(\Omega ; \mathbb{R}^{3}\right)^{*}\right)} \leqq C .
\end{aligned}
$$

From this, we see that an expression like $a-\operatorname{div} \omega+\operatorname{div}^{2} \mathfrak{h}$ in the "dual" norm in (118b) refers to the linear functional 


$$
\begin{aligned}
z \mapsto & \int_{Q} a \cdot z+\omega: \mathrm{E}(z)+\mathfrak{h} \vdots \nabla \mathrm{E}(z) \mathrm{d} x \mathrm{~d} t \\
& +\int_{\Sigma}\left((\omega+\operatorname{div} \mathfrak{h}) \cdot v+\operatorname{div}^{\mathrm{s}}(\mathfrak{h} \cdot v)-\left(\operatorname{div}^{\mathrm{s}} v\right)(\mathfrak{h}:(v \otimes v))\right) \cdot z \\
& -(\mathfrak{h}:(v \otimes v)) \cdot \frac{\partial z}{\partial v} \mathrm{~d} S \mathrm{~d} t .
\end{aligned}
$$

Proof of Lemma 3. These "dual" estimates ((118)a-c) can be obtained routinely as a consequence of the previously derived ones. In particular, in view of (119) and (101a), the estimate (118b) turns into the estimate for

$$
\begin{aligned}
& \sup _{\|v\|_{L^{2}\left(I ; W^{2,2}\left(\Omega ; \mathbb{R}^{3}\right)\right) \leqq 1}} \int_{Q}\left(\sigma_{\mathrm{E}}\left(\overline{\mathfrak{q}}_{\tau}, \bar{\vartheta}_{\tau}\right)+\mathbb{D} \mathrm{E}\left(\dot{u}_{\tau}\right)+\chi_{\mathrm{E}}^{\prime}\left(\underline{\mathfrak{q}}_{\tau}\right)^{\top} \bar{\omega}_{\tau}\right): \mathrm{E}(v) \\
& +\left(\mathbb{C}_{\mathrm{hpr}} \nabla \mathrm{E}\left(\bar{u}_{\tau}\right)+\mathbb{D}_{\mathrm{hpr}} \nabla \mathrm{E}\left(\dot{u}_{\tau}\right)\right) \vdots \nabla \mathrm{E}(v) \\
& +\left(\mu_{0}\left(\nabla h_{\tau}\right)^{\top} m_{\tau}-\bar{f}_{\tau}\right) \cdot v \mathrm{~d} x \mathrm{~d} t+\int_{\Sigma} \bar{g}_{\tau} \cdot v \mathrm{~d} S \mathrm{~d} t \\
& =\sup _{\|v\|_{L^{2}\left(I ; W^{2,2}\left(\Omega ; \mathbb{R}^{3}\right)\right) \leqq 1}} \int_{Q}\left(\sigma_{\mathrm{E}}\left(\overline{\mathfrak{q}}_{\tau}, \bar{\vartheta}_{\tau}\right)+\mathbb{D} \mathrm{E}\left(\dot{u}_{\tau}\right)+\chi_{\mathrm{E}}^{\prime}\left(\underline{\mathfrak{q}}_{\tau}\right)^{\top} \bar{\omega}_{\tau}\right): \mathrm{E}(v) \\
& +\left(\mathbb{C}_{\mathrm{hpr}} \nabla \mathrm{E}\left(\bar{u}_{\tau}\right)+\mathbb{D}_{\mathrm{hpr}} \nabla \mathrm{E}\left(\dot{u}_{\tau}\right)\right) \vdots \nabla \mathrm{E}(v) \\
& -\mu_{0}\left((\operatorname{div} v) \bar{m}_{\tau}+\nabla \bar{m}_{\tau} v\right) \cdot \bar{h}_{\tau}-\bar{f}_{\tau} \cdot v \mathrm{~d} x \mathrm{~d} t \\
& +\int_{\Sigma} \bar{g}_{\tau} \cdot v+\mu_{0}(m \cdot h)(v \cdot n) \mathrm{d} S \mathrm{~d} t
\end{aligned}
$$

where (35) has been used with $v$ in place of $\dot{u}$. Similarly, the estimate (118c) is required to bound

$$
\sup _{\|v\|_{L^{\infty}\left(I ; W^{3,2}\left(\Omega ; \mathbb{R}^{3}\right)\right) \leqq 1}} \int_{Q}\left(\mathscr{K}_{0}\left(\overline{\mathfrak{q}}_{\tau}, \bar{\vartheta}_{\tau}\right) \nabla \bar{\vartheta}_{\tau}+\mathscr{K}\left(\overline{\mathfrak{q}}_{\tau}, \bar{\vartheta}_{\tau}\right): \nabla \overline{\mathfrak{q}}_{\tau}\right) \cdot \nabla v-\bar{r}_{\tau} v \mathrm{~d} x \mathrm{~d} t,
$$

with $\bar{r}_{\tau}$ from (115). Using (102a-g), boundedness of both (120) and (121) then becomes obvious. Due to the assumptions (72b) and (72h), we have, for instance:

$$
\begin{aligned}
& \int_{Q} \sigma_{\mathrm{E}}\left(\overline{\mathfrak{q}}_{\tau}, \bar{\vartheta}_{\tau}\right): \mathrm{E}(v) \mathrm{d} x \mathrm{~d} t \\
& \quad \leqq\left\|\sigma_{\mathrm{E}}\left(\overline{\mathfrak{q}}_{\tau}, \bar{\vartheta}_{\tau}\right)\right\|_{L^{2}\left(I ; L^{6 / 5}\left(\Omega ; \mathbb{R}_{\mathrm{sym}}^{3 \times 3}\right)\right)}\|\mathrm{E}(v)\|_{L^{2}\left(I ; L^{6}\left(\Omega ; \mathbb{R}_{\mathrm{sym}}^{3 \times 3}\right)\right)}
\end{aligned}
$$

where

$$
\begin{aligned}
\left\|\sigma_{\mathrm{E}}\left(\overline{\mathfrak{q}}_{\tau}, \bar{\vartheta}_{\tau}\right)\right\|_{L^{2}\left(I ; L^{6 / 5}\right)} \leqq & C\left(1+\left\|\bar{m}_{\tau}^{k}\right\|_{L^{\infty}\left(I ; L^{6}\left(\Omega ; \mathbb{R}^{3}\right)\right)}\right. \\
& \left.+\left\|\overline{\mathrm{E}}_{\tau}^{k}\right\|_{L^{\infty}\left(I ; L^{6}\left(\Omega ; \mathbb{R}_{\mathrm{sym}}^{3 \times 3}\right)\right)}+\left\|\bar{\vartheta}_{\tau}^{k}\right\|_{L^{\infty}\left(I ; L^{1}(\Omega)\right)}\right),
\end{aligned}
$$


which is bounded because of (102a), (102e), and (102c). All other terms on the right-hand side of (120) can be estimated in the same manner. Notice that the term

$$
\begin{aligned}
& \int_{Q}\left(\nabla \bar{m}_{\tau} v\right) \cdot \bar{h}_{\tau} \mathrm{d} x \mathrm{~d} t \leqq C\left\|\nabla \bar{m}_{\tau}\right\|_{L^{\infty}\left(I ; L^{2}\left(\Omega ; \mathbb{R}^{3 \times 3}\right)\right)} \\
& \quad \times\left\|\bar{h}_{\tau}\right\|_{L^{\infty}\left(I ; L^{2}\left(\Omega ; \mathbb{R}^{3 \times 3}\right)\right)}\|v\|_{L^{2}\left(I ; L^{\infty}\left(\Omega ; \mathbb{R}^{3}\right)\right)}
\end{aligned}
$$

can also be estimated as

$$
\begin{aligned}
& \int_{Q}(\operatorname{div} v) \bar{m}_{\tau} \cdot \bar{h}_{\tau} \mathrm{d} x \mathrm{~d} t \leqq C\left\|\bar{m}_{\tau}\right\|_{L^{\infty}\left(I ; L^{6}\left(\Omega ; \mathbb{R}^{3}\right)\right)} \\
& \quad \times\left\|\bar{h}_{\tau}\right\|_{L^{\infty}\left(I ; L^{2}\left(\Omega ; \mathbb{R}^{3 \times 3}\right)\right)}\|\operatorname{div} v\|_{L^{2}\left(I ; L^{6}\left(\Omega ; \mathbb{R}^{3}\right)\right)}
\end{aligned}
$$

As to (118d), it follows from (77e) that the equation curl $\bar{h}_{\tau}+\tau\left|\bar{e}_{\tau}\right|^{\eta-2} \bar{e}_{\tau}=$ $-\mathscr{S}\left(\overline{\mathfrak{q}}_{\tau}, \bar{\vartheta}_{\tau}\right) \bar{e}_{\tau}$ holds in the sense of distributions, where the right-hand side has already been shown to be bounded in $L^{2}\left(Q ; \mathbb{R}^{3}\right)$.

Using $(77 \mathrm{~d})$, the norm in estimate $(118 \mathrm{e})$ means

$$
\begin{aligned}
& \left\|\dot{h}_{\tau}\right\|_{L^{2}\left(I ; L_{\text {curl }, 0}^{2}\left(\Omega ; \mathbb{R}^{3}\right)^{*}\right)}\left\langle\dot{h}_{\tau}, v\right\rangle \\
& =\sup _{\|v\|_{L^{2}\left(I ; L_{\text {curl }, 0}^{2}\left(\Omega ; \mathbb{R}^{3}\right)\right) \leqq 1}} \sup _{\|v\|_{L^{2}\left(I ; L_{\text {curl }, 0}^{2}\left(\Omega ; \mathbb{R}^{3}\right)\right) \leqq 1}\left\langle\nabla \bar{m}_{\tau} \dot{u}_{\tau}+\left(\operatorname{div} \dot{u}_{\tau}\right) \bar{m}_{\tau}+\frac{\operatorname{curl} \bar{e}_{\tau}}{\mu_{0}}+\dot{m}_{\tau}, v\right\rangle} \int_{\|v\|_{L^{2}\left(I ; L_{\operatorname{curl}, 0}^{2}\left(\Omega ; \mathbb{R}^{3}\right)\right) \leqq 1}} \int_{Q}\left(\nabla \bar{m}_{\tau} \dot{u}_{\tau}+\left(\operatorname{div} \dot{u}_{\tau}\right) \bar{m}_{\tau}+\dot{m}_{\tau}\right) \cdot v+\frac{\bar{e}_{\tau} \operatorname{curl} v}{\mu_{0}} \mathrm{~d} x \mathrm{~d} t
\end{aligned}
$$

and then (118e) follows by knowing already that $\nabla \bar{m}_{\tau} \dot{u}_{\tau} \in L^{2}\left(Q ; \mathbb{R}^{3}\right)$ since $\nabla \bar{m}_{\tau} \in L^{\infty}\left(I ; L^{2}\left(\Omega, \mathbb{R}^{3 \times 3}\right)\right)$ and $\dot{u}_{\tau} \in L^{2}\left(I ; L^{\infty}\left(\Omega, \mathbb{R}^{3}\right)\right)$, and that $\left(\operatorname{div} \dot{u}_{\tau}\right) \bar{m}_{\tau} \in$ $L^{2}\left(Q ; \mathbb{R}^{3}\right)$ since $\operatorname{div} \dot{u}_{\tau} \in L^{2}\left(I ; L^{6}(\Omega)\right)$ and $\bar{m}_{\tau} \in L^{\infty}\left(I ; L^{6}\left(\Omega, \mathbb{R}^{3}\right)\right)$, and that $\dot{m}_{\tau} \in L^{2}\left(Q ; \mathbb{R}^{3}\right)$, and also that $\bar{e}_{\tau} \in L^{2}\left(Q ; \mathbb{R}^{3}\right)$. Note that in the last equality in (122) the boundary term vanishes due to the assumed boundary condition $v \times v=0$.

Proposition 1 (Convergence for $\tau \rightarrow 0$.). Let (72) hold and let $\eta$ as used in (77) be sufficiently large as specified in Lemma 1; also, let (80) hold. Then there is a subsequence such that

$$
\begin{aligned}
& u_{\tau} \rightarrow u \text { strongly in } W^{1,2}\left(I ; W^{2,2}\left(\Omega ; \mathbb{R}^{3}\right)\right), \\
& m_{\tau} \rightarrow m \text { strongly in } W^{1,2}\left(I ; W^{1,2}\left(\Omega ; \mathbb{R}^{3}\right)\right), \\
& \bar{\vartheta}_{\tau} \rightarrow \vartheta \text { strongly in } L^{s}(Q) \text { with any } s<5 / 3, \\
& \bar{e}_{\tau} \rightarrow e \text { strongly in } L^{2}\left(Q ; \mathbb{R}^{3}\right), \\
& \bar{h}_{\tau} \rightarrow h \text { weakly* in } L^{\infty}\left(I ; L^{2}\left(\Omega ; \mathbb{R}^{3}\right)\right), \\
& \bar{\omega}_{\tau} \rightarrow \omega \text { weakly* in } L^{\infty}\left(Q ; \mathbb{R}^{L}\right),
\end{aligned}
$$


and, moreover,

$$
\begin{aligned}
& \bar{h}_{\tau}-\bar{h}_{\mathrm{b}, \tau} \rightarrow h-h_{\mathrm{b}} \text { weakly in } L^{\eta^{\prime}}\left(I ; L_{\mathrm{curl}, 0}^{2, \eta^{\prime}}\left(\Omega ; \mathbb{R}^{3}\right)\right) \text {, and } \\
& \operatorname{curl} \bar{h}_{\tau}+\tau\left|\bar{e}_{\tau}\right|^{\gamma-2} \bar{e}_{\tau} \rightarrow \operatorname{curl} h \text { weakly in } L^{2}\left(Q ; \mathbb{R}^{3 \times 3}\right) \text {. }
\end{aligned}
$$

Any $(u, m, \vartheta, h, e, \omega)$ obtained in this way is a weak solution to the system (64) with boundary/initial conditions (31b-d,f,g) and (65), which also preserves the energies as claimed in Theorem 1.

Proof. For lucidity, we divide the proof into 6 steps.

Step 1. Convergence in the semilinear mechanical/magnetic/electro part. We select a weakly* converging subsequence with respect to the norms from the estimates (102a-f) and (118). Moreover, Aubin-Lions' theorem based on (102g) and (118c) interpolated with the estimate (102c) gives the strong convergence (123c).

The equations $(64 \mathrm{a}, \mathrm{b}, \mathrm{d}, \mathrm{e})$ are obviously semilinear, and therefore the weak convergence is sufficient to obtain them in the limit from the corresponding equations (101a,b,d,e). To this goal, we again use Aubin-Lions' theorem to get, with $\left(123 \mathrm{c}\right.$ ), both $\overline{\mathfrak{q}}_{\tau} \rightarrow \mathfrak{q}$ and $\underline{\mathfrak{q}}_{\tau} \rightarrow \mathfrak{q}$ strongly to pass through the nonlinearities $\mathscr{S}\left(\overline{\mathfrak{q}}_{\tau}, \bar{\vartheta}_{\tau}\right), \chi^{\prime}\left(\underline{\mathfrak{q}}_{\tau}\right)$, and $\mathscr{K}_{i}\left(\overline{\mathfrak{q}}_{\tau}, \bar{\vartheta}_{\tau}\right)$ for $i=0,1,2$.

The quasilinear regularizing terms in (101a,b,e) vanish during the limit passage. This can be seen from the estimates $(102 \mathrm{~h}-\mathrm{j})$, for example,

$$
\begin{aligned}
\left.\left|\int_{Q} \tau\right| \mathrm{E}\left(\bar{u}_{\tau}\right)\right|^{\eta-2} \mathrm{E}\left(\bar{u}_{\tau}\right): \mathrm{E}(v) \mathrm{d} x \mathrm{~d} t \mid & \leqq \tau\left\|\mathrm{E}\left(\bar{u}_{\tau}\right)\right\|_{L^{\eta}\left(Q ; \mathbb{R}^{3 \times 3}\right)}^{\eta-1}\|\mathrm{E}(v)\|_{L^{\eta}\left(Q ; \mathbb{R}^{3 \times 3}\right)} \\
& \leqq C \tau^{1 / \eta}\|\mathrm{E}(v)\|_{L^{\eta}\left(Q ; \mathbb{R}^{3 \times 3}\right)} \rightarrow 0
\end{aligned}
$$

for any smooth $v$ with $C$ from (102h), similarly for $\tau\left|\nabla \mathrm{E}\left(\bar{u}_{\tau}\right)\right|^{\eta-2} \nabla \mathrm{E}\left(\bar{u}_{\tau}\right)$, and also for the terms $\tau\left|\bar{m}_{\tau}\right|^{\eta-2} \bar{m}_{\tau}, \tau\left|\nabla \bar{m}_{\tau}\right|^{\eta-2} \nabla \bar{m}_{\tau}$, and $\tau\left|\bar{e}_{\tau}\right|^{\eta-2} \bar{e}_{\tau}$ in (101b,e). Thus $(64 \mathrm{a}, \mathrm{b}, \mathrm{d}, \mathrm{e})$ in their weak formulation, that is, $(70 \mathrm{a}, \mathrm{b}, \mathrm{d}, \mathrm{e})$, has been proved.

Step 2. Passage to the limit in the variational inequality (101f). Writing (101f) in the weak form like (70f), we obtain

$$
\begin{aligned}
& \left.\int_{Q} \zeta(z)-\bar{\omega}_{\tau} \cdot\left(z-\chi_{\mathrm{E}}^{\prime} \underline{\mathfrak{q}}_{\tau}\right) \mathrm{E}\left(\dot{u}_{\tau}\right)-\chi_{m}^{\prime}\left(\underline{\mathfrak{q}}_{\tau}\right) \dot{m}_{\tau}\right) \mathrm{d} x \mathrm{~d} t \\
& \quad \geqq \int_{Q} \zeta\left(\chi_{\mathrm{E}}^{\prime}\left(\underline{\mathfrak{q}}_{\tau}\right) \mathrm{E}\left(\dot{u}_{\tau}\right)+\chi_{m}^{\prime}\left(\underline{\mathfrak{q}}_{\tau}\right) \dot{m}_{\tau}\right) \mathrm{d} x \mathrm{~d} t .
\end{aligned}
$$

The convergence $\bar{\omega}_{\tau}: \chi_{\mathrm{E}}^{\prime}\left(\underline{\mathfrak{q}}_{\tau}\right) \mathrm{E}\left(\dot{u}_{\tau}\right)$ weakly in $L^{2}\left(Q ; \mathbb{R}^{L}\right)$ is by using the AubinLions theorem, which also gives strong convergence of $\mathrm{E}\left(\dot{u}_{\tau}\right)$ due to the concept of viscous hyperstresses that we have used. For the convergence of the right-hand-side term, we use (strong $\times$ weak)-lower semicontinuity of $(\mathfrak{q}, \dot{\mathfrak{q}}) \mapsto$ $\xi\left(\chi_{\mathrm{E}}^{\prime}(\mathfrak{q}) \mathrm{E}(\dot{u})+\chi_{m}^{\prime}(\mathfrak{q}) \dot{m}\right)$, relying on convexity and non-negativity of $\zeta$, see $[18$, Sect. 4.3, Thm.4.4]. Thus the only difficult term is $\bar{\omega}_{\tau} \cdot \chi_{m}^{\prime}\left(\underline{\mathfrak{q}}_{\tau}\right) \dot{m}_{\tau}$ for which we can get only a limsup-estimate. 
To this goal, we choose $z=\dot{m}_{\tau}$ as the test function in (77b). By the convergence results established in Step 1, and by the weak-lower semicontinuity of the $L^{2}$ norm we obtain

$$
\begin{aligned}
& \limsup _{\tau \rightarrow 0} \int_{Q} \bar{\omega}_{\tau} \cdot \chi_{m}^{\prime}\left(\underline{\mathfrak{q}}_{\tau}\right) \dot{m}_{\tau} \mathrm{d} x \mathrm{~d} t \\
&=\limsup _{\tau \rightarrow 0} \int_{Q}\left(\chi_{m}^{\prime}\left(\underline{\mathfrak{q}}_{\tau}\right)^{\top} \bar{\omega}_{\tau}\right) \cdot \dot{m}_{\tau} \mathrm{d} x \mathrm{~d} t \\
&=\limsup _{\tau \rightarrow 0} \int_{Q}\left(\frac{\bar{m}_{\tau} \times \dot{m}_{\tau}}{\gamma\left(\left|\bar{m}_{\tau}\right|\right)}+\mu_{0} \bar{h}_{\tau}-\sigma_{m}\left(\overline{\mathfrak{q}}_{\tau}, \bar{\vartheta}_{\tau}\right)-\tau\left|\bar{m}_{\tau}\right|^{\eta-2} \bar{m}_{\tau}-\alpha \dot{m}_{\tau}\right) \cdot \dot{m}_{\tau} \\
& \quad-\left(\lambda \nabla \bar{m}_{\tau}+\tau\left|\nabla \bar{m}_{\tau}\right|^{\eta-2} \nabla \bar{m}_{\tau}\right): \nabla \dot{m}_{\tau} \mathrm{d} x \mathrm{~d} t \\
& \leqq \\
& \quad \limsup _{\tau \rightarrow 0} \int_{Q}\left(\mu_{0} \bar{h}_{\tau}-\sigma_{m}\left(\overline{\mathfrak{q}}_{\tau}, \bar{\vartheta}_{\tau}\right)-\alpha \dot{m}_{\tau}\right) \cdot \dot{m}_{\tau} \mathrm{d} x \mathrm{~d} t \\
& \quad-\liminf _{\tau \rightarrow 0} \int_{\Omega} \frac{\lambda}{2}\left|\nabla m_{\tau}(T, \cdot)\right|^{2} \mathrm{~d} x \\
& \quad+\lim _{\tau \rightarrow 0} \int_{\Omega} \frac{\lambda}{2}\left|\nabla m_{\tau}(0, \cdot)\right|^{2}+\frac{\tau}{\eta}\left|m_{\tau}(0, \cdot)\right|^{\eta}+\frac{\tau}{\eta}\left|\nabla m_{\tau}(0, \cdot)\right|^{\eta} \mathrm{d} x \\
& \leqq \int_{Q}\left(\mu_{0} h-\sigma_{m}(\mathfrak{q}, \vartheta)-\alpha \dot{m}\right) \cdot \dot{m} \mathrm{~d} x \mathrm{~d} t \\
&-\int_{\Omega} \frac{\lambda}{2}|\nabla m(T, \cdot)|^{2}-\frac{\lambda}{2}|\nabla m(0, \cdot)|^{2} \mathrm{~d} x,
\end{aligned}
$$

where the usual gyroscopic-term cancellation and (80b) have been used.

By (123a), (123b), and (123c), we have that, in particular, $\mathfrak{q}=(\mathrm{E}(u), m) \in$ $L^{\infty}\left(I ; L^{6}\left(\Omega ; \mathbb{R}^{3 \times 3} \times \mathbb{R}^{3}\right)\right)$ and $\vartheta \in L^{5 / 3-\varepsilon}(Q)$.

Moreover, by (74b),

$$
\begin{aligned}
\left\|\sigma_{m}(\mathfrak{q}, \vartheta)\right\|_{L^{2}\left(Q ; \mathbb{R}^{3}\right) \leqq} & \left\|\phi_{m}^{\prime}(\mathfrak{q})\right\|_{L^{2}\left(Q ; \mathbb{R}^{3}\right)}+\left\|\mathcal{A}_{2}(\mathfrak{q}, \vartheta)\right\|_{L^{2}\left(Q ; \mathbb{R}^{3}\right)} \\
\leqq & C\left(1+\left\||\mathfrak{q}|^{3}\right\|_{L^{2}\left(Q ; \mathbb{R}^{3}\right)}+\|\sqrt{\phi(\mathfrak{q})}\|_{L^{2}\left(Q ; \mathbb{R}^{3}\right)}\right. \\
& \left.+\|\left.\sqrt{\vartheta}\right|_{L^{2}\left(Q ; \mathbb{R}^{3}\right)}\right) \\
= & C\left(1+\|\mathfrak{q}\|_{L^{6}\left(Q ; \mathbb{R}^{3}\right)}^{3}+\|\vartheta\|_{L^{1}\left(Q ; \mathbb{R}^{3}\right)}^{1 / 2}\right) .
\end{aligned}
$$

It follows from (64b) with (72k) and (127) that $\Delta m \in L^{2}\left(Q ; \mathbb{R}^{3}\right)$; note that $|m / \gamma(|m|)| \leqq 1 / \varepsilon$ with $\varepsilon>0$ from $(72 \mathrm{k})$. Moreover, since $m \in$ $L^{2}\left(I ; W^{1,2}\left(\Omega ; \mathbb{R}^{3}\right)\right) \cap W^{1,2}\left(I ; L^{2}\left(\Omega ; \mathbb{R}^{3}\right)\right)$, the function $\bar{I} \ni t \mapsto \nabla m(t, \cdot) \in$ $L^{2}\left(\Omega ; \mathbb{R}^{3 \times 3}\right)$ is weakly continuous. Thus, the following by-parts integration formula holds:

$$
\int_{\Omega} \frac{\lambda}{2}|\nabla m(T, \cdot)|^{2}-\frac{\lambda}{2}|\nabla m(0, \cdot)|^{2} \mathrm{~d} x=\int_{Q} \lambda \Delta m \cdot \dot{m} \mathrm{~d} x \mathrm{~d} t .
$$


Combining (126), (128), and (70b) we obtain

$$
\begin{aligned}
& \limsup _{\tau \rightarrow 0} \int_{Q} \bar{\omega}_{\tau} \cdot \chi_{m}^{\prime}\left(\underline{\mathfrak{q}}_{\tau}\right) \dot{m}_{\tau} \mathrm{d} x \mathrm{~d} t \\
& \quad \leqq \int_{Q}\left(\mu_{0} h-\sigma_{m}(\mathfrak{q}, \vartheta)-\alpha \dot{m}\right) \cdot \dot{m}+\lambda \Delta m \cdot \dot{m} \mathrm{~d} x \mathrm{~d} t \\
& \quad=\int_{Q} \omega \cdot \chi_{m}^{\prime}(\mathfrak{q}) \dot{m} \mathrm{~d} x \mathrm{~d} t .
\end{aligned}
$$

Taking the limsup/liminf of both sides of (125) and using (129), we arrive at (70f). Step 3. Mechanical/magnetic energy preservation (49) with (51). We test the mechanical/magnetic/electro part (64a,b,d,e), respectively, by $\dot{u}, \dot{m}, h$, and $e$. We use (132) and (134) to make the by-part integration. Here (128) and the last equality in (129) are used once more. From the estimate (118b), it follows that

$$
\begin{aligned}
& \varrho \ddot{u}_{\tau}^{\mathrm{i}}-\tau \operatorname{div}\left(\left|\mathrm{E}\left(\bar{u}_{\tau}\right)\right|^{\eta-2} \mathrm{E}\left(\bar{u}_{\tau}\right)\right) \\
& \quad+\tau \operatorname{div}^{2}\left(\left|\nabla \mathrm{E}\left(\bar{u}_{\tau}\right)\right|^{\eta-2} \nabla \mathrm{E}\left(\bar{u}_{\tau}\right)\right) \rightarrow \zeta \in L^{2}\left(I ; W^{2,2}\left(\Omega ; \mathbb{R}^{3}\right)^{*}\right) .
\end{aligned}
$$

Testing both sides of (101a) by a smooth test function $w$ with compact support in $Q$, passing to the limit, and using the convergence to zero of the regularizing $\eta$-terms, we obtain:

$$
\begin{aligned}
\langle\zeta, w\rangle= & \lim _{\tau \rightarrow 0} \int_{Q} \varrho \dot{u}_{\tau}^{\mathrm{i}} \cdot \dot{w}-\tau\left|\mathrm{E}\left(\bar{u}_{\tau}\right)\right|^{\eta-2} \mathrm{E}\left(\bar{u}_{\tau}\right): \mathrm{E}(w) \\
& +\tau\left|\nabla \mathrm{E}\left(\bar{u}_{\tau}\right)\right|^{\eta-2} \nabla \mathrm{E}\left(\bar{u}_{\tau}\right) \vdots \nabla \mathrm{E}(w) \mathrm{d} x \mathrm{~d} t=\int_{Q} \varrho \dot{u} \cdot \dot{w} \mathrm{~d} x \mathrm{~d} t .
\end{aligned}
$$

This implies that $\zeta=\varrho \ddot{u}$ in the sense of distributions, and hence $\varrho \ddot{u}$ is in duality with $\dot{u}$ :

$$
\dot{u} \in L^{2}\left(I ; W^{2,2}\left(\Omega ; \mathbb{R}^{3}\right)\right) \quad \& \quad \varrho \ddot{u} \in L^{2}\left(I ; W^{2,2}\left(\Omega ; \mathbb{R}^{3}\right)^{*}\right) .
$$

By (130) and (131), we have

$$
\varrho \dot{u}_{\tau}(T)=\varrho \dot{u}_{0}+\int_{0}^{T} \varrho \ddot{u}_{\tau} \mathrm{d} t \rightarrow \varrho \dot{u}_{0}+\int_{0}^{T} \zeta \mathrm{d} t=\varrho \dot{u}(T)
$$

where the weak convergence is in $W^{2,2}\left(\Omega ; \mathbb{R}^{3}\right)^{*}$. Moreover, it follows from (102a) that $\varrho \dot{u}_{\tau}(T)$ is bounded in $L^{2}\left(\Omega ; \mathbb{R}^{3}\right)$. Consequently, the weak convergence (133) is actually in $L^{2}\left(\Omega ; \mathbb{R}^{3}\right)$.

Another important idea is that, although in general $\bar{h}_{\tau}-\bar{h}_{\mathrm{b}, \tau} \notin L^{2}\left(I ; L_{\text {curl }, 0}^{2}\right.$ $\left.\left(\Omega ; \mathbb{R}^{3}\right)\right)$, see $(123 \mathrm{~g})$, in the limit we have

$$
h-h_{\mathrm{b}} \in L^{2}\left(I ; L_{\text {curl }, 0}^{2}\left(\Omega ; \mathbb{R}^{3}\right)\right) \text { and } \dot{h} \in L^{2}\left(I ; L_{\text {curl }, 0}^{2}\left(\Omega ; \mathbb{R}^{3}\right)^{*}\right),
$$


because we have already proved (70e). Hence, in the sense of distributions on $Q$, we have curl $h=\mathscr{S}(\mathfrak{q}, \vartheta) e \in L^{2}\left(Q ; \mathbb{R}^{3}\right)$ and also $v \times\left.\left(h-h_{\mathrm{b}}\right)\right|_{\Sigma}=0$, which can be seen by passing to the limit in the identity

$$
\int_{\Sigma}\left(v \times\left(\bar{h}_{\tau}-\bar{h}_{\mathrm{b}, \tau}\right)\right) \cdot v \mathrm{~d} S \mathrm{~d} t=\int_{Q} \operatorname{curl}\left(\bar{h}_{\tau}-\bar{h}_{\mathrm{b}, \tau}\right) \cdot v-\left(\bar{h}_{\tau}-\bar{h}_{\mathrm{b}, \tau}\right) \cdot \operatorname{curl} v \mathrm{~d} x \mathrm{~d} t,
$$

with $\tau \rightarrow 0$ for any smooth $v$ by using (123e) and (123g), while the later information in (134) is inherited by (118e), respectively.

The contribution of the $\omega$-terms arising from (70) with $v=\dot{u}$ and (70b) integrated over $[0, T]$ with $z=\dot{m}$ can be treated as:

$$
\omega: \chi_{\mathrm{E}}^{\prime}(\mathfrak{q}) \mathrm{E}(\dot{u})+\omega \cdot \chi_{m}^{\prime}(\mathfrak{q}) \dot{m}=\omega: \frac{\partial}{\partial t} \chi(\mathfrak{q})=\zeta\left(\frac{\partial}{\partial t} \chi(\mathfrak{q})\right)
$$

Note that, by putting $z=0$ into (70f), we get the inequality $\omega:\left(\chi_{\mathrm{E}}^{\prime}(\mathfrak{q}) \mathrm{E}(\dot{u})+\right.$ $\left.\chi_{m}^{\prime}(\mathfrak{q}) \dot{m}\right) \geqq \zeta\left(\chi_{\mathrm{E}}^{\prime}(\mathfrak{q}) \mathrm{E}(\dot{u})+\chi_{m}^{\prime}(\mathfrak{q}) \dot{m}\right)$, but we need the equality for $(135)$. Here we use that, having proved (70f), we already have proved that $\omega \in L^{\infty}\left(Q ; \mathbb{R}^{L}\right)$ belongs to $\partial \zeta\left(\chi_{\mathrm{E}}^{\prime}(\mathfrak{q}) \mathrm{E}(\dot{u})+\chi_{m}^{\prime}(\mathfrak{q}) \dot{m}\right)=\partial \zeta\left(\frac{\partial}{\partial t} \chi(\mathfrak{q})\right)$. The chain rule used in the last equality in (135) holds in $L^{2}(Q)$ for any $\omega$ belonging almost everywhere to this set just due to the assumed degree- 1 homogeneity of $\zeta$; in fact, it holds if and only if $\zeta$ is degree- 1 homogeneous.

Altogether, we arrive to the energy balance (49) integrated over $I$ and employing $\Phi$ from (45), if (73) is also taken into account.

Step 4. Strong convergence of $\nabla \mathrm{E}\left(\dot{u}_{\tau}\right)$ and $\dot{m}_{\tau}$ and $\bar{e}_{\tau}$. Using (109) with $k=T / \tau$ and the abbreviation (45), we have

$$
\begin{array}{rl}
\int_{Q} & \mathbb{D}(\dot{u}): \mathrm{E}(\dot{u})+\mathbb{D}_{\mathrm{hpr}} \nabla \mathrm{E}(\dot{u}): \nabla \mathrm{E}(\dot{u})+\alpha|\dot{m}|^{2}+\mathscr{S}(\mathfrak{q}, \vartheta) e \cdot e \mathrm{~d} x \mathrm{~d} t \\
\leqq & \liminf _{\tau \rightarrow 0} \int_{Q} \mathbb{D} \mathrm{E}\left(\dot{u}_{\tau}\right): \mathrm{E}\left(\dot{u}_{\tau}\right)+\mathbb{D}_{\mathrm{hpr}} \nabla \mathrm{E}\left(\dot{u}_{\tau}\right): \nabla \mathrm{E}\left(\dot{u}_{\tau}\right) \\
& +\alpha\left|\dot{m}_{\tau}\right|^{2}+\mathscr{S}\left(\overline{\mathfrak{q}}_{\tau}, \bar{\vartheta}_{\tau}\right) \bar{e}_{\tau} \cdot \bar{e}_{\tau} \mathrm{d} x \mathrm{~d} t \\
\leqq & \limsup _{\tau \rightarrow 0} \int_{Q} \mathbb{D} \mathrm{E}\left(\dot{u}_{\tau}\right): \mathrm{E}\left(\dot{u}_{\tau}\right)+\mathbb{D}_{\mathrm{hpr}} \nabla \mathrm{E}\left(\dot{u}_{\tau}\right): \nabla \mathrm{E}\left(\dot{u}_{\tau}\right) \\
& +\alpha\left|\dot{m}_{\tau}\right|^{2}+\mathscr{S}\left(\overline{\mathfrak{q}}_{\tau}, \bar{\vartheta}_{\tau}\right) \bar{e}_{\tau} \cdot \bar{e}_{\tau} \mathrm{d} x \mathrm{~d} t \\
\leqq & \limsup _{\tau \rightarrow 0} \Phi\left(u_{0 \tau}, v_{0}, m_{0 \tau}, h_{0}\right)-\Phi\left(u_{\tau}(T), \dot{u}_{\tau}(T), m_{\tau}(T), h_{\tau}(T)\right) \\
& +\int_{\Omega} \frac{\tau}{\eta}\left|\mathrm{E}\left(u_{0 \tau}\right)\right|^{\eta}+\frac{\tau}{\eta}\left|\nabla \mathrm{E}\left(u_{0 \tau}\right)\right|^{\eta}+\frac{\tau}{\eta}\left|m_{0 \tau}\right|^{\eta} \mathrm{d} x-\int_{\Sigma} \bar{g}_{\tau} \cdot \dot{u}_{\tau} \mathrm{d} S \mathrm{~d} t \\
& +\int_{Q} \bar{f}_{\tau} \cdot \dot{u}_{\tau}-\zeta\left(\chi_{\mathrm{E}}^{\prime}\left(\mathrm{E}\left(\underline{u}_{\tau}\right), \underline{m}_{\tau}\right) \mathrm{E}\left(\dot{u}_{\tau}\right)+\chi_{m}^{\prime}\left(\mathrm{E}\left(\underline{u}_{\tau}\right), \underline{m}_{\tau}\right) \dot{m}_{\tau}\right)+\operatorname{curl} h_{\mathrm{b}, \tau} \cdot e_{\tau} \\
& +\mu_{0}\left(\dot{h}_{\tau}+\dot{m}_{\tau}-\nabla \bar{m}_{\tau} \dot{u}_{\tau}-\left(\operatorname{div} \dot{u}_{\tau}\right) \bar{m}_{\tau}\right) \cdot h_{\mathrm{b}, \tau}-\mathscr{A}\left(\overline{\mathfrak{q}}_{\tau}, \bar{\vartheta}_{\tau}\right): \dot{\mathfrak{q}}_{\tau} \mathrm{d} x \mathrm{~d} t
\end{array}
$$




$$
\begin{aligned}
\leqq & \Phi\left(u_{0}, v_{0}, m_{0}, h_{0}\right)-\Phi(u(T), \dot{u}(T), m(T), h(T))-\int_{\Sigma} g \cdot \dot{u} \mathrm{~d} S \mathrm{~d} t \\
& +\int_{Q} f \cdot \dot{u}-\zeta\left(\chi_{\mathrm{E}}^{\prime}(\mathrm{E}(u), m) \mathrm{E}(\dot{u})+\chi_{m}^{\prime}(\mathrm{E}(u), m) \dot{m}\right)+\operatorname{curl} h_{\mathrm{b}} \cdot e \\
& +\mu_{0}(\dot{h}+\dot{m}-\nabla m \dot{u}-(\operatorname{div} \dot{u}) m) \cdot h_{\mathrm{b}}-\mathscr{A}(\mathfrak{q}, \vartheta): \dot{\mathfrak{q}} \mathrm{d} x \mathrm{~d} t \\
= & \int_{Q} \mathbb{D} \mathrm{E}(\dot{u}): \mathrm{E}(\dot{u})+\mathbb{D}_{\mathrm{hpr}} \nabla \mathrm{E}(\dot{u}): \nabla \mathrm{E}(\dot{u})+\alpha|\dot{m}|^{2}+\mathscr{S}(\mathfrak{q}, \vartheta) e \cdot e \mathrm{~d} x \mathrm{~d} t .
\end{aligned}
$$

For the first inequality in (136), we used that $\int_{Q} \mathscr{S}(\mathfrak{q}, \vartheta) e \cdot e \mathrm{~d} x \mathrm{~d} t \leqq \lim \inf _{\tau \rightarrow 0}$ $\int_{Q} \mathscr{S}\left(\overline{\mathfrak{q}}_{\tau}, \bar{\vartheta}_{\tau}\right) \bar{e}_{\tau} \cdot \bar{e}_{\tau} \mathrm{d} x \mathrm{~d} t$ by using the strong convergence $\left(\overline{\mathfrak{q}}_{\tau}, \bar{\vartheta}_{\tau}\right) \rightarrow(\mathfrak{q}, \vartheta)$ in $L^{1}\left(Q ; \mathbb{R}^{3}\right)$ by Aubin-Lions theorem, along with continuity and positive semidefiniteness of $\mathscr{S}$, see again [18, Sect. 4.3, Thm.4.4]. Later in (136), we used the weak upper-semicontinuity of $-\Phi$ and of the term $-\zeta(\cdot)$. We also used (80). The last equality in (136) was just proved in Step 3.

Altogether, we can see that equalities hold everywhere in (136). Denoting $\mathfrak{e}=$ $(\mathrm{E}(\dot{u}), \nabla \mathrm{E}(\dot{u}), \dot{m}, e),(136)$ can be written more "compactly" as

$$
\lim _{\tau \rightarrow 0} \int_{Q} \mathfrak{S}\left(\overline{\mathfrak{q}}_{\tau}, \bar{\vartheta}_{\tau}\right) \overline{\mathfrak{e}}_{\tau}: \overline{\mathfrak{e}}_{\tau} \mathrm{d} x \mathrm{~d} t=\int_{Q} \mathfrak{S}(\mathfrak{q}, \vartheta) \dot{\mathfrak{e}}: \mathfrak{e} \mathrm{d} x \mathrm{~d} t,
$$

with the shorthand notation $\mathfrak{S}(\mathfrak{q}, \vartheta):=\left(\mathbb{D}, \mathbb{D}_{\mathrm{hpr}}, \alpha \mathbb{I}, \mathscr{S}(\mathfrak{q}, \vartheta)\right)$. Then, with $c_{\mathfrak{S}}$ denoting the positive-definiteness constant of $\mathfrak{S}$, we have

$$
\begin{aligned}
c_{\mathfrak{S}} \lim _{\tau \rightarrow 0}\left\|\overline{\mathfrak{e}}_{\tau}-\mathfrak{e}\right\|^{2} & \leqq \limsup _{\tau \rightarrow 0} \int_{Q} \mathfrak{S}\left(\overline{\mathfrak{q}}_{\tau}, \bar{\vartheta}_{\tau}\right)\left(\overline{\mathfrak{e}}_{\tau}-\mathfrak{e}\right):\left(\overline{\mathfrak{e}}_{\tau}-\mathfrak{e}\right) \mathrm{d} x \mathrm{~d} t \\
& =\limsup _{\tau \rightarrow 0} \int_{Q} \mathfrak{S}\left(\overline{\mathfrak{q}}_{\tau}, \bar{\vartheta}_{\tau}\right) \overline{\mathfrak{e}}_{\tau}: \overline{\overline{\mathfrak{e}}}_{\tau}+\mathfrak{S}\left(\overline{\mathfrak{q}}_{\tau}, \bar{\vartheta}_{\tau}\right) \mathfrak{e}:\left(\mathfrak{e}-2 \overline{\mathfrak{e}}_{\tau}\right) \mathrm{d} x \mathrm{~d} t \\
& =\int_{Q} \mathfrak{S}(\mathfrak{q}, \vartheta) \mathfrak{e}: \mathfrak{e}+\mathfrak{S}(\mathfrak{q}, \vartheta) \mathfrak{e}:(\mathfrak{e}-2 \mathfrak{e}) \mathrm{d} x \mathrm{~d} t=0
\end{aligned}
$$

where, successively, we used (137) and the continuity of the Nemytskii mapping $\left(\overline{\mathfrak{q}}_{\tau}, \bar{\vartheta}_{\tau}\right) \mapsto \mathfrak{S}\left(\overline{\mathfrak{q}}_{\tau}, \bar{\vartheta}_{\tau}\right) \mathfrak{e}$ into an $L^{2}(Q)$-space, and eventually the weak convergence $\overline{\mathfrak{e}}_{\tau} \rightarrow \mathfrak{e}$ in the respective $L^{2}(Q)$-space. Obviously, (138) yields the strong convergence $\overline{\mathfrak{e}}_{\tau} \rightarrow \mathfrak{e}$, that is (123a,b,d).

Step 5. Limit passage in the heat equation (101c). Having proved the strong convergence in Step 4, the right-hand side of (101c) converges strongly in $L^{1}(Q)$ and this limit passage is then easy.

Step 6. Total energy preservation (46) with (51). Now that we have $\dot{\vartheta} \in$ $L^{1}\left(I ; W^{3,2}(\Omega)^{*}\right)$, see $(118 \mathrm{c})$, and having realizes the already proved identity $(64 \mathrm{c})$, which is in duality with the constant 1 , we can perform this test rigorously and sum it with the mechanical/magnetic energy balance already obtained in Step 3.

Acknowledgements. The authors are thankful to Drs. Michal Landa, Petr Sedlák, and Petr Šittner for many inspiring discussions, as well as to two anonymous referees for several useful suggestions that led to improvement of the explanation of complicated physical aspects. 


\section{References}

1. Albanese, R., Rubinacci, G.: Formulation of the eddy-current problem. IEE Proc. A 137, 16-22 (1990)

2. Arndt, M., Griebel, M., Novák, V., Roubíček, T., Šittner, P.: Martensitic/austenitic transformation in NiMnGa: simulation and experimental approaches. Int. J. Plasticity 22, 1943-1961 (2006)

3. Arndt, M., Griebel, M., Roubíček, T.: Modelling and numerical simulation of martensitic transformation in shape memory alloys. Cont. Mech. Thermodyn. 15, 463-485 (2003)

4. BALl, J.M., JAMES, R.D.: Proposed experimental tests of a theory of fine microstructure and the two-well problem. Phil. Trans. R. Soc. Lond. A 338, 389-450 (1992)

5. Bertotti G.: Hysteresis in Magnetism. Academic Press, San Diego, 1998

6. Bertsch, M., Podio-Guidugli, P., Valente, V.: On the dynamics of deformable ferromagnets. I. Global weak solutions for soft ferromagnets at rest. Ann. Mat. Pura Appl. 179, 331-360 (2001)

7. Boccardo, L., Dall'aglio, A., Gallouët, T., Orsina, L.: Nonlinear parabolic equations with measure data. J. Funct. Anal. 147, 237-258 (1997)

8. BocCardo, L., GallouËt, T.: Non-linear elliptic and parabolic equations involving measure data. J. Funct. Anal. 87, 149-69 (1989)

9. Bossavit, A.: Computational Electromagnetism. Academic Press, San Diego, 1998

10. BRÉZIS, H.: Équations et inéquations non-linéaires dans les espaces vectoriel en dualité. Ann. Inst. Fourier 18, 115-176 (1968)

11. Brown, W.F.: Micromagnetics. Krieger Publishing Co., New York, 1963

12. Duvaut, G., Lions, J.L.: Les Inéquations en Méchanique et en Physique. Dunod, Paris, 1972

13. DeSimone, A.: Energy minimizers for large ferromagnetic bodies. Arch. Rational Mech. Anal. 125, 99-143 (1996)

14. DeSimone, A., Kohn, R., Müller, S., Otto, F.: Two-dimensional modelling of soft ferromagnetic films. Proc. R. Soc. Lond. A 457, 2983-2991 (2001)

15. DeSimone, A., Podio-Guidugli, P.: On the continuum theory of deformable ferromagnetic solids. Arch. Rational Mech. Anal. 136, 201-233 (1996)

16. Faulkner, M.G., Amalraj, J.J., Bhattacharyya, A.: Experimental determination of thermal and electrical properties of NiTi shape memory wires. Smart Mater. Struct. 9, 632-639 (2000)

17. Gilbert, T.: A phenomenological theory of damping in ferromagnetic materials. IEEE Trans. Mag. 40, 3443-3449 (2004)

18. GiUsti, E.: Direct Methods in the Calculus of Variations. World Scientific, New Yersey, 2003

19. Gori, F., Carnevale, D., Doro Altan, A., Nicosia, S., Pennestrì, E.: A new hysteretic behavior in the electrical resistivity of flexinol shape memory alloys versus temperature. Int. J. Thermophys. 27, 866-879 (2006)

20. Gurtin, M.E., Murdoch, A.I.: A continuum theory of elastic material surfaces. Arch. Rational Mech. Anal. 57, 291-323 (1974)

21. He, Z., Gall, K.R., Brinson, L.C.: Use of electric reistance testing to redefine the transformation kinetics and phase diagram for shape-memory alloys. Metall. Mater. Trans. A 37A, 579-581 (2006)

22. Heczko, O., Seiner, H., Sedlák, P., Kopeček, J., Landa, M.: Anomalous lattice softening of Ni2MnGa austenite due to magnetoelastic coupling. J. Appl. Phys. 111, 07A929 (2012)

23. James, R.D., Wuttig, M.: Magnetostriction of Martensite. Philos. Mag. A 77, $1273-$ 1299 (1998)

24. Krevet, B., Kohl, M.: Thermodynamic Modelling of Ferromagnetic Shape Memory Actuators. Mat. Sci. Forum 635, 175-180 (2010) 
25. LANDIS, C.M.: A continuum thermodynamics formulation for micro-magnetomechanics with applications to ferromagnetic shape memory alloys. J. Mech. Phys. Solids 56, 3059-3076 (2008)

26. Melcher, A., Nestler, B.: A phase-field model for magnetic shape memory alloys: Microstructure evolution under the influence of elastic and magnetic forces. Proc. Appl. Math. Mech. 8, 10745-10746 (2008)

27. Murray, S.J., Marioni, M., Tello, P.G., Allen, S.M., O’Handley, R.C.: Giant magnetic-field-induced strain in Ni-Mn-Ga crystals: experimental results and modeling. J. Magn. Magn. Mater. 226-230 945-947 (2001)

28. Novák, V., Šittner, P., Dayananda, G.N., Braz-Fernandes, F.M., Mahesh, K.K.: Electric resistance variation of NiTi shape memory alloy wires in thermomechanical tests: experiments and simulation. Mater. Sci. Eng. A 481-482, 127-133 (2008)

29. Onsager, L.: Reciprocal relations in irreversible processes. Phys. Rev. II 37, 405-426 (1931)

30. Onsager, L.: Reciprocal relations in irreversible processes. Phys. Rev. II 38 2265-2279 (1931)

31. ÖtTInger, H.C.: Beyond Equilibrium Thermodynamics. Wiley, Hoboken, NJ, 2002

32. Plecháč, P., Roubíček, T.: Visco-elasto-plastic model for martensitic phase transformation in shape-memory alloys. Math. Method Appl. Sci. 25, 1281-1298 (2002)

33. Podio-Guidugli, P.: Contact interactions, stress, and material symmetry, for nonsimple elastic materials. Theor. Appl. Mech. 28-29, 261-276 (2002)

34. Podio-Guidugli, P., Vianello, M.: Hypertractions and hyperstresses convey the same mechanical information. Cont. Mech. Thermodyn. 22, 163-176 (2010)

35. Podio-Guidugli, P., Roubíček, T., Tomassetti, G.: A thermodynamically-consistent theory of the ferro/paramagnetic transition. Arch. Rational Mech. Anal. 198, 1057-1094 (2010)

36. Rajagopal, K.R., Roubíček, T.: On the effect of dissipation in shape-memory alloys. Nonlinear Anal. Real World Appl. 4, 581-597 (2003)

37. Roubíčex, T.: Nonlinear Partial Differential Equations with Applications. Birkhäuser, Basel, 2005 (2nd ed. 2012).

38. Roubíček, T.: Nonlinearly coupled thermo-visco-elasticity. Nonlinear Differ. Equ. Appl. (2013, to appear)

39. Roubíček, T., Tomassetti, G.: Thermodynamics of shape-memory alloys under electric current. Zeit. Angew. Math. Phys. 61, 1-20 (2010)

40. Roubíček, T., Tomassetti, G.: Ferromagnets with eddy currents and pinning effects: their thermodynamics and analysis. Math. Mod. Meth. Appl. Sci. 21, 29-55 (2011)

41. Roubíček, T., Tomassetti, G., Zanini, C.: The Gilbert equation with dry-friction-type damping. J. Math. Anal. Appl. 355, 453-468 (2009)

42. Seiner, H., Bicanová, L., Sedlák, P., Landa, M., Heller, L., Aaltio, I.: Magnetoelastic attenuation in austenitic phase of Ni-Mn-Ga alloy investigated by ultrasonic methods. Mat. Sci. Eng. A, 521-522, 205-208 (2009)

43. Seiner, H., Heczko, O., Sedlák, P., Bodnárová, L., Novotný M., Kopeček, J., LANDA, M.: Combined effect of structural softening and magneto-elastic coupling on elastic coefficients of Ni Mn Ga austenite. J. Alloys Compounds (2012, to appear)

44. ŠILhavÝ, M.: Phase transitions in non-simple bodies. Arch. Rational Mech. Anal. 88 (1985), 135-161

45. Sozinov, A., Likhachev, A.A., Lanska, N., Ullakko, K.: Giant magnetic-fieldinduced strain in NiMnGa seven-layered martensitic phase. Appl. Phys. Lett. 10, 1746$1748(2002)$

46. Tickle, R., James, R.D.: Magnetic and magnetomechanical properties of Ni2MnGa. J. Magn. Magn. Mater. 195, 627-638 (1999)

47. Toupin, R.A.: Elastic materials with couple stresses. Arch. Rational Mech. Anal. 11, 385-414 (1962) 
48. Uchil, J., Mahesh, K.K., Ganesh Kumara, K.: Electrical resistivity and strain recovery studies on the effect of thermal cycling under constant strass on R-phase in NiTi shape memory alloy. Phys. B 324, 419-428 (2002)

49. Visintin, A.: Modified Landau-Lifshitz equation for ferromagnetism. Phys. B 233, 365-369 (1997)

50. Worgull, J., Petti, E., Trivisonno, J.: Behavior of the elastic properties near an intermediate phase transition in $\mathrm{Ni}_{2}$ MnGa. Phys. Rev. B 54, 15695-15699 (1996)

51. Wu, X.D., FAN, Y.Z., Wu, J.S.: A study on the variations of the eletrical resistance for NiTi shape memory alloy wires during the thermomechanical loading. Mater. Design 21, 511-515 (2000)

52. Zayak, A.T., Buchelnikov, V.D., Entel, P.: A Ginzburg-Landau theory for Ni-MnGa. Phase Trans. 75, 243-256 (2002)

Mathematical Institute Charles University

Sokolovská 83, 18675 Praha 8, Czech Republic

and

Institute of Thermomechanics of the ASCR Dolejškova 5, 18200 Praha 8, Czech Republic. e-mail: tomas.roubicek@mff.cuni.cz

and

Dipartimento di Ingegneria Civile Università di Roma "Tor Vergata" Via Politecnico 1, Rome 00133, Italy. e-mail: tomassetti@ing.uniroma2.it

(Received March 20, 2012 / Accepted August 15, 2012)

Published online June 18, 2013 - (C) Springer-Verlag Berlin Heidelberg (2013) 